\title{
Counting closed geodesics in strata
}

\author{
Alex Eskin ${ }^{1} \cdot$ Maryam Mirzakhani $^{2}$. \\ Kasra Rafi ${ }^{3}$
}

Received: 21 June 2013 / Accepted: 30 September 2018

(C) Springer-Verlag GmbH Germany, part of Springer Nature 2018

\begin{abstract}
We compute the asymptotic growth rate of the number $N(\mathcal{C}, R)$ of closed geodesics of length $\leq R$ in a connected component $\mathcal{C}$ of a stratum of quadratic differentials. We prove that, for any $0 \leq \theta \leq 1$, the number of closed geodesics $\gamma$ of length at most $R$ such that $\gamma$ spends at least $\theta$-fraction of its time outside of a compact subset of $\mathcal{C}$ is exponentially smaller than $N(\mathcal{C}, R)$. The theorem follows from a lattice counting statement. For points $x, y$ in the moduli space $\mathcal{M}(S)$ of Riemann surfaces, and for $0 \leq \theta \leq 1$ we find an upperbound for the number of geodesic paths of length $\leq R$ in $\mathcal{C}$ which connect a point near $x$ to a point near $y$ and spend at least a $\theta$-fraction of the time outside of a compact subset of $\mathcal{C}$.
\end{abstract}

Deceased: Maryam Mirzakhani.

$凶$ Kasra Rafi

rafi@math.toronto.edu

http://www.math.toronto.edu/ rafi/

Alex Eskin

eskin@math.uchicago.edu

http://math.uchicago.edu/ eskin/

Maryam Mirzakhani

mmirzakh@math.stanford.edu

1 Department of Mathematics, University of Chicago, Chicago, IL 60637, USA

2 Department of Mathematics, Stanford University, Building 380, Stanford, CA 94305 , USA

3 Department of Mathematics, University of Toronto, Toronto ON, M5S 2E4, Canada 


\section{Introduction}

Let $S=S_{g, p}$ be a surface of genus $g$ with $p$ punctures and let $\mathcal{M}(S)$ be the moduli space of Riemann surfaces homeomorphic to $S$. The co-tangent bundle of $\mathcal{M}(S)$ is naturally identified with $\mathcal{Q M}(S)$ the space of finite area quadratic differentials on $S$. Let $\mathcal{Q}^{1} \mathcal{M}(S)$ be subspace of quadratic differentials of area 1 . There is a natural $\operatorname{SL}(2, \mathbb{R})$ action on the $\mathcal{Q}^{1} \mathcal{M}(S)$. The orbits of the diagonal flow, $g_{t}=\left[\begin{array}{cc}e^{t} & 0 \\ 0 & e^{-t}\end{array}\right]$, projects to geodesics in $\mathcal{M}(S)$ equipped with the Teichmüller metric. For $R>0$, let $N(R)$ be the number of closed Teichmüller geodesics of length less than or equal to $R$ on $\mathcal{Q}^{1} \mathcal{M}(S)$. It was shown in [16] that, as $R \rightarrow \infty$, the number $N(R)$ is asymptotic to $e^{h R} / h R$, where $h=6 g-6+2 p$.

The moduli space of quadratic differentials is naturally stratified: to each quadratic differential $(x, q) \in \mathcal{Q M}(S)$ we can associate $\sigma(q)=$ $\left(v_{i}, \ldots, v_{k}, \varsigma\right)$ where $v_{1}, \ldots, v_{k}$ are the orders of the zeros and poles of $q$, and $\varsigma \in\{-1,1\}$ is equal to 1 if $q$ is the square of an abelian differential and -1 otherwise. For a given tuple $\sigma$, we say a quadratic differential $(x, q) \in \mathcal{Q M}(S)$ is of type $\sigma$ if $\sigma(q)=\sigma$. The space $\mathcal{Q M}(\sigma)$ of all quadratic differentials in $\mathcal{Q M}(S)$ of type $\sigma$ is called the stratum of quadratic differentials of type $\sigma$. The stratum $\mathcal{Q M}(\sigma)$ is an analytic orbifold of real dimension $4 g+2 k+\varsigma-3$.

Let $\mathcal{Q}^{1} \mathcal{M}(\sigma)$ be the space of quadratic differentials in $\mathcal{Q M}(\sigma)$ of area 1. It is not necessarily connected (see $[26,29]$ for the classification of the connected components), however, each connected component is $\operatorname{SL}(2, \mathbb{R})$ invariant. Let $\mathcal{C}$ be a connected component of $\mathcal{Q}^{1} \mathcal{M}(\sigma)$. In this paper, we study the asymptotic growth rate of the number $N(\mathcal{C}, R)$ of closed Teichmüller geodesics of length less than or equal to $R$ in $\mathcal{C}$. Our main tool is estimating the number $N\left(\mathcal{C}_{\mathcal{K}}, R\right)$ of closed geodesics that stay completely outside of a large compact set $\mathcal{K} \subset \mathcal{C}$.

Theorem 1.1 Given $\delta>0$ there exists a compact subset $\mathcal{K} \subset \mathcal{C}$ and $R_{0}>0$ such that for all $R>R_{0}$,

$$
N\left(\mathcal{C}_{\mathcal{K}}, R\right) \leq e^{(h-1+\delta) R} .
$$

This result implies that:

Theorem 1.2 As $R \rightarrow \infty$, we have

$$
N(\mathcal{C}, R) \sim \frac{e^{h R}}{h R},
$$

where $h=\frac{1}{2}\left[1+\operatorname{dim}_{\mathbb{R}}(\mathcal{C})\right]$ and the notation $A \sim B$ means that the ratio $A / B$ tends to 1 as $R$ tends to infinity. 
Remark 1.3 In the case of abelian differentials, $h$ is equal to the dimension of the relative homology of $S$ with respect to the set of singular points of $(x, q) \in \mathcal{C}$, otherwise $h$ is one less.

Recurrence of geodesics We prove a stronger version of Theorem 1.1. Every quadratic differential defines a singular Euclidean metric on the surface $S$ and for every compact set $\mathcal{K} \subset \mathcal{C}$, there is a lower bound for the $q$-length of a saddle connection where $q \in \mathcal{K}$. Here, we restrict attention to closed geodesics where more than one simple closed curve or saddle connection is assumed to be short; in this case the growth rate is of even lower order.

Let $\mathcal{T}(S)$ be the Teichmüller space, the universal cover of $\mathcal{M}(S)$. Let $\mathcal{Q T}(S)$ and $\mathcal{Q}^{1} \mathcal{T}(S)$ be defined similarly. To distinguish between points in the Moduli space and Teichmüller space, we use $x \in \mathcal{M}(S)$ and $X \in \mathcal{T}(S)$. Also, we use the notation $(x, q)$ for points in $\mathcal{Q}^{1} \mathcal{M}(S)$ and $(X, q)$ for points in $\mathcal{Q}^{1} \mathcal{T}(S)$. We denote a geodesic in $\mathcal{Q}^{1} \mathcal{M}(S)$ by $\mathbf{g}$ and a geodesic in $\mathcal{Q}^{1} \mathcal{T}(S)$ by $\mathcal{G}$. The space $\mathcal{Q}^{1} \mathcal{T}(S)$ is also naturally stratified. We denote the space of quadratic differentials in $\mathcal{Q}^{1} \mathcal{T}(S)$ of type $\sigma$ by $\mathcal{Q}^{1} \mathcal{T}(\sigma)$. To simplify the notation, let

$$
\mathcal{Q}(\sigma):=\mathcal{Q}^{1} \mathcal{T}(\sigma)
$$

Recall that $\operatorname{Ext}_{X}(\alpha)$ denotes the extremal length of a a simple closed curve $\alpha$ on the Riemann surface $X \in \mathcal{T}(S)$. (see Eq. (1) for definition). We introduce a notion of extremal length for saddle connections on quadratic differentials. Essentially, the extremal length of a saddle connection $\omega$ in a quadratic differential $(X, q) \in \mathcal{Q}^{1} \mathcal{T}(S)$ with distinct end points $p_{1}$ and $p_{2}$ is the extremal length of the associated curve in the ramified double cover of $X$ with simple ramification points at only $p_{1}$ and $p_{2}$ (see Sect. 3.5 for more details).

Definition 1.4 For $\epsilon>0$ and for any quadratic differential $(X, q) \in \mathcal{Q}(\sigma)$, let $\Omega_{q}(\epsilon)$ be the set of saddle connections $\omega$ so that either $\operatorname{Ext}_{q}(\omega) \leq \epsilon$ or $\omega$ appears in a geodesic representative of a simple closed curve $\alpha$ with $\operatorname{Ext}_{X}(\alpha) \leq \epsilon$. Let $\mathcal{Q}_{j, \epsilon}(\sigma)$ be the set of quadratic differentials $(X, q)$ of type $\sigma$ so that $\Omega_{q}(\epsilon)$ contains at least $j$ disjoint homologically independent saddle connections. When $\sigma$ is fixed, we denote this set simply by $\mathcal{Q}_{j, \epsilon}$. Let $\mathcal{C}_{j, \epsilon}$ be the set of points in $\mathcal{C}$ whose lift to $\mathcal{Q}^{1} \mathcal{T}(S)$ lies in $\mathcal{Q}_{j, \epsilon}$. For $0 \leq \theta \leq 1$, define $N_{\theta}\left(\mathcal{C}_{j, \epsilon}, R\right)$ to be the number of closed geodesics of length at most $R$ in $\mathcal{C}$ that spend at least $\theta$-fraction of their length in $\mathcal{C}_{j, \epsilon}$.

In this paper, we show:

Theorem 1.5 Given $\delta>0$, there exist $\epsilon>0$ small enough and $R>0$ large enough so that, for all $j \geq 1$ and $0 \leq \theta \leq 1$,

$$
N_{\theta}\left(\mathcal{C}_{j, \epsilon}, R\right) \leq e^{(h-j \theta+\delta) R} .
$$




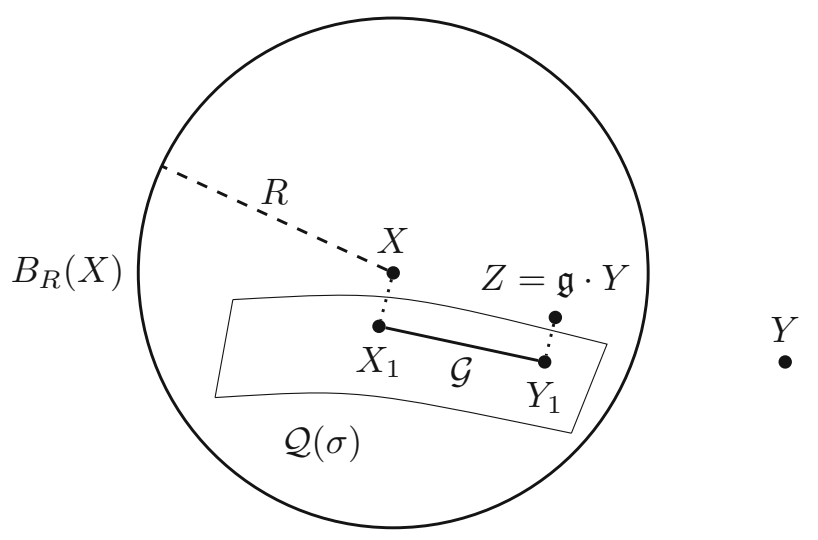

Fig. 1 There is a geodesic $\mathcal{G}$ in $\mathcal{Q}(\sigma)$ connecting a point near $X$ to a point near $Z \in B_{R}(X)$ that is in the orbit of $Y$

Remark 1.6 The condition on $\Omega_{q}(\epsilon)$ is necessary. Just assuming there are $j$ saddle connections of $q$-length less than $\epsilon$ does not reduce the exponent by $j$. In fact, for any $\epsilon$, there is a closed geodesic $\mathbf{g}$ in $\mathcal{Q}^{1} \mathcal{M}(S)$ where the number of saddle connections with $q$-length less than $\epsilon$ is as large as desired at every quadratic differential $(X, q)$ along $\mathbf{g}$. This is because the Euclidean size of a subsurface could be as small as desired (see Sect.3.4) and short saddle connection can intersect.

Lattice counting in Teichmüller space Let $\Gamma(S)$ denote the mapping class group of $S$ and let $B_{R}(X)$ denote the ball of radius $R$ in the Teichmüller space with respect to the Teichmüller metric centered at the point $X \in \mathcal{T}(S)$. It is known ([5]) that, for and $Y \in \mathcal{T}(S)$,

$$
\left|\Gamma(S) \cdot Y \cap B_{R}(X)\right| \sim \Lambda^{2} e^{(6 g-6) R},
$$

as $R \rightarrow \infty$. Here $\Lambda$ is a constant depending only on the topology of $S$ (see [13]).

The main theorem in this paper is a partial generalization of this result for the strata of quadratic differentials. Here we are interested in the case where the Teichmüller geodesic joining $X$ to $\mathfrak{g} \cdot Y$, for $\mathfrak{g} \in \Gamma(S)$, is assumed to belong to the stratum $\mathcal{Q}(\sigma)$ or stay close to it.

More precisely, for a fix $r_{0}>0$ (see Sect. 6.2), let $N_{\theta}\left(\mathcal{Q}_{j, \epsilon}, X, Y, R\right)$ be the number of points $Z \in \mathcal{T}(S)$ such that (see Fig. 1):

- $Z \in B_{R}(X)$ and $Z=\mathfrak{g} \cdot Y$, for some $\mathfrak{g} \in \Gamma(S)$.

- there is a Teichmüller geodesic segment $\mathcal{G} \subset \mathcal{Q}(\sigma)$ joining $X_{1} \in B_{r_{0}}(X)$ to $Y_{1} \in B_{r_{0}}(Z)$

- $\mathcal{G}$ spends at least $\theta$-fraction of the time in $\mathcal{Q}_{j, \epsilon}$. 
Also, for a fix $\epsilon_{0}$ (see Sect.1.3 below), we define $\mathcal{S}_{X}$ to be the set of $\epsilon_{0}$-short curves in $X$ and

$$
G(X)=1+\prod_{\beta \in \mathcal{S}_{X}} \frac{1}{\sqrt{\operatorname{Ext}_{X}(\beta)}} .
$$

Theorem 1.7 Given $\delta>0$, there exist $\epsilon>0$ small enough and $R>0$ large enough such that, for every $0 \leq \theta \leq 1, j \geq 1$ and $X, Y \in \mathcal{T}(S)$, we have

$$
N_{\theta}\left(\mathcal{Q}_{j, \epsilon}, X, Y, R\right) \leq G(X) G(Y) e^{(h-j \theta+\delta) R},
$$

Compare with Theorem 7.2 in [16].

\subsection{Notes on the proof}

1. Each stratum $\mathcal{Q}^{1} \mathcal{M}(\sigma)$ has an affine integral structure, and carries a unique probability measure $\mu$, called the Masur-Veech measure, invariant by the Teichmüller flow which is equivalent to the Lebesgue measure. Moreover, the restriction of the Teichmüller flow to any connected component $\mathcal{C}$ of $\mathcal{Q}^{1} \mathcal{M}(\sigma)$ is mixing with respect to the Lebesgue measure class $[32,48]$. In fact, the Teichmüller flow on $\mathcal{C}$ is exponentially mixing with respect to $\mu[2,3]$. However, we will only use the mixing property (as stated in Theorem 2.4) in this paper. 2. The main difficulty for proving Theorem 1.2 is the fact that the Teichmüller flow is not uniformly hyperbolic. As in [16], we show that the Teichmüller geodesic flow (or more precisely an associated random walk) is biased toward the part of $\mathcal{C}$ that does not contain short saddle connections (see Lemma 6.4). Similar method has been used in [16] where it is enough to use Minsky's product region theorem (see Sect. 2.5) to prove the necessary estimates. In this paper, since the projection map from $\mathcal{C}$ to $\mathcal{M}(S)$ is not easy to understand, we need different and more delicate methods to obtain similar results.

3. We define a notion of a $(q, \tau)$-regular triangulation for a quadratic differential $(X, q)$ (Definition 3.11). Such a triangulation captures the geometry of singular Euclidean metric associated to $q$ in a way that is compatible with the hyperbolic metric associated to $X$. We will show that a set of disjoint saddle connections in $\Omega_{q}(\epsilon)$ can be included in a $(q, \tau)$-regular triangulation (Lemma 3.13).

4. In order to prove Theorem 5.1 (Sect. 5) we compute, given the triangulation $T_{a}$, the number of possible triangulations $T_{b}$ which have certain bounds on their intersection number with $T_{a}$. It turns out that the number of possible triangulations $T_{b}$ is related to the number of edges in $T_{a}$ that are homologically independent. This is the main reason that the growth rate of $N_{\theta}\left(\mathcal{Q}_{j, \epsilon}, X, Y, R\right)$ is related to $\operatorname{dim}_{\mathbb{R}} \mathcal{C}$. In Sect. 3 we establish the basic properties of a $(q, \tau)$ - 
regular triangulation and in Sect. 4 we establish the necessary bounds on the intersection number between $T_{a}$ and $T_{b}$ needed in Sect. 5 .

5. Theorems 1.1 and 1.5 are essentially corollaries of Theorem 1.7. In Sect. 6, we use Theorem 5.1 to prove Theorem 1.7. Here we describe the steps involved in the proof of Theorem 1.7. First, we fix a net $\mathcal{N}$ in $\mathcal{M}(S)$ and its lift $\tilde{\mathcal{N}}$ in $\mathcal{T}(S)$. For any constant $\tau$, we note that $N_{\theta}\left(\mathcal{Q}_{j, \epsilon}, X, Y, R\right)$ is bounded above by the number of trajectories $\left\{\lambda_{0}, \ldots, \lambda_{n}\right\}$ in $\tilde{\mathcal{N}}$ from $X$ to $Y$ so that the distance between $\lambda_{i}$ and $\lambda_{i+1}$ is at most $\tau$ and, for $\theta$ proportion of steps, the segment $\left[\lambda_{i}, \lambda_{i+1}\right]$ can be approximated by a path in $\mathcal{Q}_{j, \epsilon}$.

Given $\lambda_{i}$, we bound the number of possible choices for $\lambda_{i+1}$ so that the segment $\left[\lambda_{i}, \lambda_{i+1}\right]$ can be approximated by a path in $\mathcal{Q}_{j, \epsilon}$. The bound depends on the geometry of $\lambda_{i}$ (captured by the function $G(\cdot)$.

On the other hand, if $\mathcal{G}:[a, b] \rightarrow \mathcal{Q}_{j, \epsilon}$ is a geodesic segment with initial and terminal quadratic differentials $\left(X_{a}, q_{a}\right)$ and $\left(X_{b}, q_{b}\right)$ with $|b-a| \leq \tau$, one can find a $\left(q_{a}, \tau\right)$-regular triangulation $T_{a}$ and $\left(q_{b}, \tau\right)$-regular triangulation $T_{b}$ so that $T_{a}$ and $T_{b}$ have $j$ nomologically independent edges in common (see Lemma 6.1 for the precise statement). Then Theorem 5.1 shows that the number of choices for $\lambda_{i+1}$ is also reduced by a factor $e^{-j \tau}$.

6. To obtain Theorem 1.2, we use the basic properties of the Hodge norm [5] to prove a closing lemma for the Teichmüller geodesic flow in Sect. 8. We remark that the Hodge norm behaves badly near smaller strata, i.e. near points with degenerating zeros of the quadratic differential, where quadratic differentials have small geodesic segments.

On the other hand, the set of quadratic differentials with no small geodesic segment is compact and in any compact subset of $\mathcal{C}$, the geodesic flow is uniformly hyperbolic (see $[19,48]$ and Sect. 7). Also, in view of Theorem 1.5, for any $0 \leq \theta \leq 1$, the number of closed geodesics $\gamma$ of length at most $R$ such that $\gamma$ spends at least a $\theta$-fraction of the time outside of a compact subset of $\mathcal{C}$ is exponentially smaller than $N(\mathcal{C}, R)$. Therefore, "most" closed geodesics spend at least $(1-\theta)$-fraction of the time away from the degenerating locus. This allows us to prove Theorem 1.2 following the ideas from Margulis' thesis [30].

\subsection{Further remarks and references}

1. According to the Nielsen-Thurston classification, every irreducible mapping class $\mathfrak{g} \in \Gamma(S)$ of infinite order has a representative which is a pseudo-Anosov homeomorphism. Let $K_{\mathfrak{g}}$ denote the dilatation factor of $\mathfrak{g}$ [46]. By a theorem of Bers, every closed geodesic in $\mathcal{M}(S)$ is the unique loop of minimal length in its homotopy class. Also a pseudo-Anosov $\mathfrak{g} \in \Gamma(S)$ gives rise to a closed geodesic $\mathcal{G}_{\mathfrak{g}}$ of length $\log \left(K_{\mathfrak{g}}\right)$ in $\mathcal{Q}^{1} \mathcal{M}(S)$. Hence $\log \left(K_{\mathfrak{g}}\right)$ is the translation length of $\mathfrak{g}$ as an isometry of $\mathcal{T}(S)$ [9]. In other words, 


$$
\mathcal{L}(S)=\left\{\log \left(K_{\mathfrak{g}}\right) \mid \mathfrak{g} \in \Gamma(S) \text { pseudo-Anosov }\right\}
$$

is the length spectrum of $\mathcal{M}(S)$ equipped with the Teichmüller metric. By [1] and [25], $\mathcal{L}(S)$ is a discrete subset of $\mathbb{R}$. Hence the number of conjugacy classes of pseudo-Anosov elements of the group $\Gamma(S)$ with dilatation factor $K_{\mathfrak{g}} \leq e^{R}$ is finite. We remark that for any pseudo-Anosov $\mathfrak{g} \in \Gamma(S)$ the number $K_{\mathfrak{g}}$ is an algebraic number and $\log \left(K_{\mathfrak{g}}\right)$ is equal to the minimal topological entropy of any element in the same homotopy class [18]. (See [10,39] for simple explicit constructions of pseudo-Anosov mapping classes.) In terms of this notation, $N(\mathcal{C}, R)$ is the number of conjugacy classes of pseudo-Anosov elements $\mathfrak{g}$ in the mapping class group $\Gamma(S)$ with expansion factor of at most $e^{R}$ such that $\mathcal{G}_{\mathfrak{g}} \subset \mathcal{C}$.

2. The first results on this problem are due to Veech [48]. He proved that there exists a constant $c$ such that

$$
h \leq \liminf _{R \rightarrow \infty} \frac{\log N(R)}{R} \leq \limsup _{R \rightarrow \infty} \frac{\log N(R)}{R} \leq c
$$

and conjectured that $c=h$.

Foliations fixed by pseudo-Anosov maps can be characterized by being representable by eventually periodic "convergent" words [37]. Moreover, there is an inequality relating the length of the repeating part of the word corresponding to a pseudo-Anosov foliation and the dilatation factor of a pseudo-Anosov map preserving that foliation [38]. However, the estimates obtained using these inequalities are weaker.

3. The basic idea behind the proof of the main theorem in this paper is proving recurrence results for Teichmüller geodesics. Variations on this theme have been used in [4,14-16]. One reason the proof is different from [16] is that in general the projection map $\pi: \mathcal{Q}^{1} \mathcal{M}(\sigma) \rightarrow \mathcal{M}(S)$ is far from being a fibration: in many cases $\operatorname{dim}\left(\mathcal{Q}^{1} \mathcal{M}(\sigma)\right)<\operatorname{dim}(\mathcal{M}(S))$ and $\operatorname{dim}\left(\pi^{-1}(X) \cap\right.$ $\left.\mathcal{Q}^{1} \mathcal{M}(\sigma)\right)$ depends on the geometry of $X$. In this paper, we need to analyze the geometry of quadratic differentials more carefully. The results obtained in Sect. 3 allow us to deal with this issue.

4. Our results are complimentary to the following result:

Theorem 1.8 (Hammenstadt) There exists a compact $\mathcal{K} \subset \mathcal{C}$ such that for $R$ sufficiently large,

$$
N\left(\mathcal{C}_{\mathcal{K}}, R\right) \geq e^{(h-1) R}
$$

Also, by results in [22] the normalized geodesic flow invariant measure supported on the set of closed geodesics of length $\leq R$ in $\mathcal{C}$ become equidistributetd with respect to the Lebesgue measure $\mu$ as $R \rightarrow \infty$. 


\subsection{Choosing constants}

We choose our constants as follows: We call a curve short if its extremal length is less than $\epsilon_{0}$. This is a constant that depends on the topology of $S$ only (a uniform constant) and is chosen so that Theorem 2.2 and the estimate in Eq. (5) hold. We call any other constant that depends in the topology of $S$ or the choice of $\epsilon_{0}$ a uniform constant. Most of these constants are hidden in notations $\stackrel{*}{\longleftarrow}$ and $\stackrel{*}{\prec}$ (see the notation section below). For the arguments in Sect. 6 to work, we need to choose $\tau$ large enough depending on the value of $\delta$ (see proofs of Theorem 1.5 and Lemma 6.4 in Sect. 6). Then $\epsilon$ is chosen small enough depending on the value of $\tau$. We need $\epsilon \leq \epsilon_{1}=\epsilon_{1}(\tau)$ so that Lemma 3.13 holds and $\epsilon \leq \epsilon_{2}=\epsilon_{2}(\tau)$ so that Lemma 6.1 holds. The dependence on the choice of $\tau$ and $\epsilon$ is always highlighted and a constant that we call uniform does not depend on $\epsilon$ or $\tau$.

\subsection{Notation}

In this paper, the expression $\mathbf{A} \stackrel{*}{\prec} \mathbf{B}$ means that $\mathbf{A}<c \mathbf{B}$ and $\mathbf{A} \stackrel{+}{\prec} \mathbf{B}$ means $\mathbf{A} \leq \mathbf{B}+c$ for some uniform constant $c$ which only depends on the topology of $S$ (a uniform constant). We write $\mathbf{A} \stackrel{*}{ }$ B if we have both $\mathbf{A} \stackrel{*}{\prec} \mathbf{B}$ and $\mathbf{B} \stackrel{*}{\prec} \mathbf{A}$. Similarly, $\mathbf{A} \doteq \mathbf{B}$ if both $\mathbf{A} \stackrel{+}{\prec} \mathbf{C}$ and $\mathbf{B} \stackrel{+}{\prec} \mathbf{A}$ hold. The notation $\mathbf{A}=O(\mathbf{B})$ means that $\mathbf{A} \stackrel{*}{\prec} \mathbf{B}$.

\section{Teichmüller space and quadratic differentials}

In this section, we recall some definitions and known results about the geometry of $\mathcal{M}(S)$ equipped with the Teichmüller metric. For more details, see [17,24, 45].

\subsection{Teichmüller space}

Let $S$ be a connected oriented surface of genus $g$ with $p$ marked points. A point in the Teichmüller space $\mathcal{T}(S)$ is a Riemann surface $X$ of genus $g$ with $p$ marked points equipped with a diffeomorphism $f: S \rightarrow X$ sending marked points to marked points. The map $f$ provides a marking on $X$ by $S$. Two marked surfaces $f_{1}: S \rightarrow X$ and $f_{2}: S \rightarrow Y$ define the same point in $\mathcal{T}(S)$ if and only if $f_{1} \circ f_{2}^{-1}: Y \rightarrow X$ is isotopic (relative to the marked points) to a holomorphic map. By the uniformization theorem, each point $X$ in $\mathcal{T}(S)$ has a complete metric of constant curvature -1 with punctures at the marked points. The space $\mathcal{T}(S)$ is a complex manifold of dimension $3 g-3+p$, diffeomorphic to a cell. Let $\Gamma(S)$ denote the mapping class group of $S$, the group of isotopy 
classes of orientation preserving self-homeomorphisms of $S$ fixing the marked points point-wise. The mapping class group $\Gamma(S)$ acts on $\mathcal{T}(S)$ by changing the marking. The quotient space

$$
\mathcal{M}(S)=\mathcal{T}(S) / \Gamma(S)
$$

is the moduli space of Riemann surfaces homeomorphic to $S$.

\subsection{Teichmüller distance and Teichmüller's theorem}

The Teichmüller metric on $\mathcal{T}(S)$ is defined by

$$
d_{\mathcal{T}}\left(\left(f_{1}: S \rightarrow X_{1}\right),\left(f_{2}: S \rightarrow X_{2}\right)\right)=\frac{1}{2} \inf _{f} \log \left(K_{f}\right)
$$

where $f: X_{1} \rightarrow X_{2}$ ranges over all quasiconformal maps isotopic to $f_{1} \circ f_{2}^{-1}$ and $K_{f} \geq 1$ is the dilatation of $f$. For convenience, we will often omit the marking and write $X \in \mathcal{T}(S)$. To distinguish between a marked point and an un-marked point, we use small case letters for points in Moduli space and write $x \in \mathcal{M}(S)$.

We recall the following important theorem due to Teichmüller. Given any $X_{1}, X_{2} \in \mathcal{T}(S)$, there exists a unique quasi-conformal map $f$, called the Teichmüller map and quadratic differentials $\left(X_{i}, q_{i}\right) \in \mathcal{Q}^{1}\left(X_{i}\right)$ such that the map $f$ takes zeroes and poles of $q_{1}$ to zeroes and poles of $q_{2}$ of the same order and $d_{\mathcal{T}}\left(X_{1}, X_{2}\right)=\frac{1}{2} \log \left(K_{f}\right)$.

\subsection{The space of quadratic differentials}

Let $\mathcal{Q}(X)$ denote the vector space of quadratic differentials on $X$ with at most simple poles at the marked points of $X$. The cotangent space of $\mathcal{T}(S)$ at a point $X$ can be identified with $\mathcal{Q}(X)$ and the space

$$
\mathcal{Q T}(S)=\{(X, q) \mid X \in \mathcal{T}(S), q \in Q(X)\}
$$

can be identified with the cotangent space of $\mathcal{T}(S)$.

In local coordinates $z, q$ is the tensor given by $q(z) d z^{2}$, where $q(z)$ is a meromorphic function with poles of degree at most one at the punctures of $X$. In this setting, the Teichmüller metric corresponds to the norm

$$
\|q\|_{\mathcal{T}}=\int_{X}|q(z)||d z|^{2}
$$


on $\mathcal{Q T}(S)$. Let $\mathcal{Q}^{1} \mathcal{T}(S)$ denote the space of (marked) unit area quadratic differentials, or equivalently the unit cotangent bundle over $\mathcal{T}(S)$. Define

$$
\mathcal{Q M}(S) \cong \mathcal{Q T}(S) / \Gamma(S) \text { and } \mathcal{Q}^{1} \mathcal{M}(S) \cong \mathcal{Q}^{1} \mathcal{T}(S) / \Gamma(S)
$$

To simplify the notation, in this paper, we let $\mathbf{p}$ denote both projection maps

$$
\mathbf{p}: \mathcal{T}(S) \rightarrow \mathcal{M}(S), \quad \text { and } \quad \mathbf{p}: \mathcal{Q}^{1} \mathcal{T}(S) \rightarrow \mathcal{Q}^{1} \mathcal{M}(S) .
$$

Similarly, $\pi$ will denote both projection maps:

$$
\pi: \mathcal{Q}^{1} \mathcal{M}(S) \rightarrow \mathcal{M}(S), \quad \text { and } \quad \pi: \mathcal{Q}^{1} \mathcal{T}(S) \rightarrow \mathcal{T}(S) .
$$

\subsection{Extremal and hyperbolic lengths of simple closed curves}

By a curve we always mean the free homotopy class of a non-trivial, nonperipheral, simple closed curve on the surface $S$ where the homotopy is relative to the marked points. We denote the set of curves on $S$ by $\mathcal{S}$ to emphasize that they are simple curves.

Given a curve $\alpha$ on the surface $S$ and $X \in \mathcal{T}(S)$, let $\ell_{X}(\alpha)$ denote the hyperbolic length of the unique geodesic in the homotopy class of $\alpha$ on $X$. The extremal length of a curve $\alpha$ on $X$ is defined by

$$
\operatorname{Ext}_{X}(\alpha):=\sup _{\rho} \frac{\ell_{\rho}(\alpha)^{2}}{\operatorname{Area}(X, \rho)},
$$

where the supremum is taken over all metrics $\rho$ conformally equivalent to $X$, and $\ell_{\rho}(\alpha)$ denotes the infimum of $\rho$-lengths of representatives of $\alpha$.

Here $X$ can be any Riemann surface, even an open annulus. Recall that the modulus of an annulus $A$ is defined to

$$
\operatorname{Mod}(A):=\frac{1}{\operatorname{Ext}_{A}(\alpha)},
$$

where $\alpha$ is the core curve of $A$.

Given curves $\alpha$ and $\beta$ on $S$, the intersection number $\mathrm{i}(\alpha, \beta)$ is the minimum number of points in which representatives of $\alpha$ and $\beta$ must intersect. In general, by [20]

$$
\mathrm{i}(\alpha, \beta) \leq \sqrt{\operatorname{Ext}_{X}(\alpha)} \cdot \sqrt{\operatorname{Ext}_{X}(\beta)} .
$$

The following result [28] relates the ratios of extremal lengths to the Teichmüller distance: 
Theorem 2.1 (Kerckhoff) Given $X, Y \in \mathcal{T}(S)$, the Teichmüller distance between $X$ and $Y$ is given by

$$
d_{\mathcal{T}}(X, Y)=\sup _{\beta \in \mathcal{S}} \log \left(\frac{\sqrt{\operatorname{Ext}_{X}(\beta)}}{\sqrt{\operatorname{Ext}_{Y}(\beta)}}\right) .
$$

The relationship between the extremal length and the hyperbolic length is complicated; in general, by the definition of extremal length,

$$
\frac{\ell_{X}(\alpha)^{2}}{\pi(2 g-2+p)} \leq \operatorname{Ext}_{X}(\alpha)
$$

Also, for any $X \in \mathcal{T}(S)$, the extremal length can be extended continuously to the space of measured laminations [28] such that

$$
\operatorname{Ext}_{X}(r \cdot \lambda)=r^{2} \operatorname{Ext}_{X}(\lambda)
$$

As a result, since the space of projectivized measured laminations is compact, for every $X$ there exists a constant $C_{X}$ so that

$$
\frac{1}{c_{X}} \ell_{X}(\alpha) \leq \sqrt{\operatorname{Ext}_{X}(\alpha)} \leq c_{X} \ell_{X}(\alpha) .
$$

However, by [31]

$$
\frac{1}{\pi} \leq \frac{\operatorname{Ext}_{X}(\alpha)}{\ell_{X}(\alpha)} \leq \frac{1}{2} e^{\ell_{X}(\alpha) / 2}
$$

Hence, as $\ell_{X}(\alpha) \rightarrow 0$,

$$
\frac{\ell_{X}(\alpha)}{\operatorname{Ext}_{X}(\alpha)} \stackrel{*}{`} 1
$$

\subsection{Minsky's product theorem}

Let $\mathcal{A}=\left\{\alpha_{1}, \ldots, \alpha_{j}\right\}$ be a collection of disjoint simple closed curves on $S$ and, for a fixed $\epsilon_{0}$,

$$
\mathcal{T}_{\epsilon_{0}}(\mathcal{A})=\left\{X \in \mathcal{T}(S) \mid \operatorname{Ext}_{X}\left(\alpha_{i}\right) \leq \epsilon_{0}, \quad 1 \leq i \leq j\right\} .
$$

Then, using the Fenchel-Nielsen coordinates on $\mathcal{T}(S)$, we can define

$$
\Phi_{\mathcal{A}}: \mathcal{T}_{\epsilon_{0}}(\mathcal{A}) \rightarrow\left(\mathbb{H}^{2}\right)^{j}
$$


by

$$
\Phi_{\mathcal{A}}(X)=\left(\theta_{1}(X), \frac{1}{\ell_{X}\left(\alpha_{1}\right)}, \ldots, \theta_{j}(X), \frac{1}{\ell_{X}\left(\alpha_{j}\right)}\right)
$$

Here, $\theta_{i}(\cdot)$ is the Fenchel-Nielson twist coordinate around $\alpha_{i}$ and represents the $x$-coordinate in upper-half plane $\mathbb{H}$ and the $y$-coordinate in $\mathbb{H}$ is the reciprocal of the hyperbolic length. Following Minsky, we get a map

$$
\Phi: \mathcal{T}_{\epsilon_{0}}(\mathcal{A}) \rightarrow\left(\mathbb{H}^{2}\right)^{j} \times \mathcal{T}(S \backslash \mathcal{A})
$$

where $\mathcal{T}(S \backslash \mathcal{A})$ is the quotient Teichmüller space obtained by collapsing all the $\alpha_{i}$. The product region theorem [36] states that for sufficiently small $\epsilon_{0}$ the Teichmüller metric on $\mathcal{T}_{\epsilon_{0}}(\mathcal{A})$ is within an additive constant of the supremum metric on $\left(\mathbb{H}^{2}\right)^{j} \times \mathcal{T}(S \backslash \mathcal{A})$. More precisely, let $d_{\mathcal{A}}(\cdot, \cdot)$ denote the supremum metric on $\left(\mathbb{H}^{2}\right)^{j} \times \mathcal{T}(S \backslash \mathcal{A})$. Then:

Theorem 2.2 (Minsky) There is $\epsilon_{0}>0$ is small enough and $B>0$ depending only on $S$ such that for all $X, Y \in \mathcal{T}_{\epsilon_{0}}(\mathcal{A})$,

$$
\left|d_{\mathcal{T}}(X, Y)-d_{\mathcal{A}}(\Phi(X), \Phi(Y))\right|<B
$$

As mentioned in the introduction, we fix $\epsilon_{0}$ so that the above theorem and the estimate in Eq. (5) hold.

\subsection{Short curves on a surface}

For $\epsilon_{0}$ as above, we say a curve $\alpha$ is short on $X$ if $\operatorname{Ext}_{X}(\alpha) \leq \epsilon_{0}$. From discussions in [36], we know that, if two curves are short in $X$ they can not intersect. Let $\mathcal{S}_{X}$ be the set of short curves on $X$. Define $G: \mathcal{T}(S) \rightarrow \mathbb{R}_{+}$by

$$
G(X)=1+\prod_{\alpha \in \mathcal{S}_{X}} \frac{1}{\sqrt{\operatorname{Ext}_{X}(\alpha)}}
$$

If $d_{\mathcal{T}}(X, Y)=O(1)$ then $G(X) \stackrel{*}{\asymp} G(Y)$. The function $G$ is $\Gamma(S)$ invariant and induces a proper function on $\mathcal{M}(S)$. We also recall the following lemma which, for example, follows from [16].

Lemma 2.3 For any $X \in \mathcal{T}(S)$ let

$$
I_{X}=\left\{\mathfrak{g} \in \Gamma(S) \mid d_{\mathcal{T}}(\mathfrak{g} \cdot X, X)=O(1)\right\} .
$$


be the set of mapping classes that move $X$ by a bounded amount. Then

$$
\left|I_{X}\right| \stackrel{*}{`} G(X)^{2} .
$$

\subsection{Stratum of quadratic differentials}

Although the value of $q \in \mathcal{Q}(X)$ at a point in $X$ depends on the local coordinates, the zero set of $q$ is well defined. As a result, there is a natural stratification of the space $\mathcal{Q T}(S)$ by the multiplicities of zeros of $q$. For $\sigma=\left(v_{1}, \ldots, v_{k}, \varsigma\right)$ define $\mathcal{Q T}(\sigma) \subset \mathcal{Q T}(S)$ to be the subset consisting of pairs $(X, q)$ of quadratic differentials on $X$ with zeros and poles of multiplicities $\left(v_{1}, \ldots, v_{k}\right)$. The poles are always assumed to be simple and are located at the marked points, however, not all marked points have to be poles. The sign $\varsigma \in\{-1,1\}$ is equal to 1 if $q$ is the square of an abelian differential (an abelian differential). Otherwise, $\varsigma=-1$. Then

$$
\mathcal{Q T}(S)=\bigsqcup_{\sigma} \mathcal{Q T}(\sigma)
$$

It is known that each $\mathcal{Q T}(\sigma)$ is an orbifold. See [32,35] for more details.

\subsection{Flat lengths of simple closed curves and saddle connections}

Let $(X, q)$ be a quadratic differential. If we represent $q$ locally as $q(z) d z^{2}$ then $|q|=|q(z)|^{\frac{1}{2}}|d z|$ defines a singular Euclidean metric on $X$ with cone points at zeros and poles. The total angle at a singular point of degree $v$ is $(2+v) \pi$. (for more details, see [45]). This is not a complete metric space since poles are a finite distance away. However, one can still talk about the geodesic representative of a curve that may pass through the poles even though the poles. Namely, for a $\operatorname{arc}$ in $(X, q)$, consider the lift of this arc to the universal cover, take the geodesic representative in the completion of the universal cover and then project it back to $(X, q)$. Following the discussion in [40, Page 185], we can ignore this issue and treat these special geodesics as we would any other geodesic.

The homotopy class of an arc (relative to its endpoints) has a unique $q$-geodesic representative. Any curve $\alpha$ either has a unique $q$-geodesic representative or there is flat cylinder of parallel representatives. In this case, we say $\alpha$ is a cylinder curve and we denote the cylinder of geodesics representatives of $\alpha$ by $F_{\alpha}$. We denote the Euclidean length of the $q$-representative of $\alpha$ by $\ell_{q}(\alpha)$. 
A saddle connection on $(X, q)$ is a $q$-geodesic segment which connects a pair of singular points without passing through one in its interior. We denote the Euclidean length of a saddle connection $\omega$ on $q$ by $\ell_{q}(\omega)$.

\subsection{Period coordinates on the strata}

In general, any saddle connection $\omega$ joining two zeros of a quadratic differential $q=\zeta d z^{2}$ determines a complex number $\operatorname{hol}_{q}(\omega)$ (after choosing a branch of $\sqrt{\zeta}$ and an orientation of $\omega$ ) by

$$
\operatorname{hol}_{q}(\omega)=\left(\int_{\omega} \Re \sqrt{\zeta}\right)+\left(\int_{\omega} \operatorname{Im} \sqrt{\zeta}\right) i
$$

We recall that for any $\sigma=\left(v_{i}, \ldots, v_{k}, \varsigma\right)$ the period coordinates gives $\mathcal{Q T}(\sigma)$ the structure of an affine manifold. Consider the first relative homology group $H_{1}(S, \Sigma, \mathbb{R})$ of the pair $(S, \Sigma)$ with $|\Sigma|=k$. Let

$$
h=(2 g+k-1)=\operatorname{dim}\left(H_{1}(S, \Sigma, \mathbb{R})\right)
$$

if $\varsigma=1$, and

$$
h=(2 g+k-2)=\operatorname{dim}\left(H_{1}(S, \Sigma, \mathbb{R})\right)-1
$$

if $\varsigma=-1$. We recall that given $(X, q) \in \mathcal{Q}^{1} \mathcal{T}(\sigma)$ there is a triangulation $T$ of the underlying surface by saddle connections (see for example [49, Proposition 3.1] and [47, Proposition 3.1]). One can choose $h$ directed edges $\omega_{1}, \ldots, \omega_{h}$ of $T$, and an open neighborhood $U_{q} \subset \mathcal{Q T}(\sigma)$ of $q$ such that the map

$$
\boldsymbol{\psi}_{T, q}: \mathcal{Q T}(\sigma) \rightarrow \mathbb{C}^{h} \quad \text { defined by } \quad \boldsymbol{\psi}_{T, q}(q)=\left(\operatorname{hol}_{q}\left(\omega_{i}\right)\right)_{i=1}^{h}
$$

is a local homeomorphism. For any other geodesic triangulation $T^{\prime}$, the map $\boldsymbol{\psi}_{T^{\prime}, q} \circ \boldsymbol{\psi}_{T, q}^{-1}$ is linear.

In case of abelian differentials $(\varsigma=1)$ it is enough to choose a basis for $H_{1}(S, \Sigma, \mathbb{R})$ from the edges of $T$. Note that for non-orientable differentials $(\varsigma=-1)$ there will be a linear relation between the holonomies of the vectors corresponding to a basis for the relative homology (see Sect. 4.3). In this case, it is enough to choose $\operatorname{dim}\left(H_{1}(S, \Sigma, \mathbb{R})\right)-1$ independent vectors of $T$. For a more detailed discussion of the holonomy coordinates see [34]. 


\subsection{Teichmüller geodesic flow}

We recall that when $3 g+p>4$ the Teichmüller metric is not even Riemannian. However, geodesics in this metric are well understood. A quadratic differential $(X, q) \in \mathcal{Q}^{1} \mathcal{T}(S)$ with zeros at $p_{1}, \ldots p_{k}$ is determined by an atlas of charts mapping open subsets of $S-\left\{p_{1}, \ldots, p_{k}\right\}$ to $\mathbb{R}^{2}$ such that the change of coordinates are of the form $v \rightarrow \pm v+c$. Therefore the group $\operatorname{SL}(2, \mathbb{R})$ acts naturally on $\mathcal{Q}^{1} \mathcal{T}(S)$ by acting on the corresponding atlas; given $A \in$ $\operatorname{SL}(2, \mathbb{R}), A \cdot q \in \mathcal{Q}^{1} \mathcal{T}(S)$ is determined by the new atlas $\left\{A \phi_{i}\right\}$. The action of the diagonal subgroup $g_{t}=\left[\begin{array}{cc}e^{t} & 0 \\ 0 & e^{-t}\end{array}\right]$ is the Teichmüller geodesic flow for the Teichmüller metric. In other words, in holonomy coordinates the Teichmüller flow is simply defined by

$$
\Re\left(\operatorname{hol}_{g_{t}(q)}\left(\omega_{i}\right)\right)=e^{t} \Re\left(\operatorname{hol}_{q}\left(\omega_{i}\right)\right),
$$

and

$$
\Im\left(\operatorname{hol}_{g_{t} q}\left(\omega_{i}\right)\right)=e^{-t} \Im\left(\operatorname{hol}_{q}\left(\omega_{i}\right)\right) .
$$

This action descends to $\mathcal{Q}^{1} \mathcal{M}(S)$ via the projection map p: $\mathcal{Q}^{1} \mathcal{T}(S) \rightarrow$ $\mathcal{Q}^{1} \mathcal{M}(S)$. We denote both actions (on $\mathcal{Q}^{1} \mathcal{T}(S)$ and $\mathcal{Q}^{1} \mathcal{M}(S)$ ) by $g_{t}$. The subspaces $\mathcal{Q}^{1} \mathcal{T}(\sigma)$ and $\mathcal{Q}^{1} \mathcal{M}(\sigma)$ are invariant under the Teichmüller geodesic flow. Moreover, we have $[32,48]$ :

Theorem 2.4 (Veech-Masur) Each connected component $\mathcal{C}$ of a stratum $\mathcal{Q}^{1} \mathcal{M}(\sigma)$ carries a unique probability measure $\mu$ in the Lebesgue measure class such that:

- the action of $\mathrm{SL}(2, \mathbb{R})$ is volume preserving and ergodic;

- Teichmüller geodesic flow is mixing.

\section{Geometry of a quadratic differential}

In this section, we recall some of the basic geometric properties of a quadratic differential $(X, q)$. We describe how the extremal length of a curve, which can be calculated from the conformal structure of $X$, relates to the singular Euclidean metric associated to $(X, q)$. We also define the notion of a $(q, \tau)$-regular triangulation, where $\tau>0$ is a large constant. This is a partial triangulation of $(X, q)$ using the saddle connections that captures the geometry of the singular Euclidean metric associated to $q$. The main statement of the section is Lemma 3.13 which shows the existence of such triangulations. In the rest of the section, we establish some basic properties of $(q, \tau)$-regular triangulations which are used in Sect. 5 . 


\subsection{Intersection number}

In the hyperbolic metric of $X$, the geodesic representatives of any two curves $\alpha$ and $\beta$ intersect minimally. Hence, the geometric intersection number between homotopy classes of curves is equal to the intersection number between their geodesic representatives.

In the singular Euclidean metric $|q|$, this is not true. First, as mentioned in 2.8 , the geodesic representative might pass through the poles even though the poles are removed from the surface. Also, the $q$-geodesic representatives of curves $\alpha$ and $\beta$ that have geometric intersection number zero may intersect. However, these intersections are tangential. That is, $\alpha$ and $\beta$ may share an edge, but they do not cross. By this, we mean that any lifts $\tilde{\alpha}$ and $\tilde{\beta}$ to the universal cover $\tilde{q}$ of $q$ have end points in the boundary that do no interlock. To simplify the exposition, when we say $\alpha$ and $\beta$ intersect, we always mean that they have an essential intersection not tangential.

We also talk about the intersection number between two saddle connections. Here, we say two saddle connections are disjoint if they have disjoint interiors or if they are equal. The intersection number between two saddle connections is the number of interior intersection points. The intersection number between a saddle connection and itself is zero. In both cases, (saddle connections and curves) the intersection number is denoted by $\mathrm{i}(\cdot, \cdot)$.

If $A$ is an embedded annulus, we distinguish between a curve $\alpha$ intersecting $A$ and crossing it. To intersect $A, \alpha$ needs only to enter the interior of $A$. The curve $\alpha$ crosses $A$ if $\alpha$ enters one side of $A$ and exits the other. To be more precise, in the annular cover $\tilde{X}_{A}$ of $X$ associated to $A$, there is a lift of $\alpha$ connecting the two boundary components of $\tilde{X}_{A}$.

\subsection{Extremal lengths and flat lengths of simple closed curves}

One can give an estimate for the extremal length of a simple closed curve $\alpha$ in $X$ by examining the singular Euclidean metric $|q|$. As mentioned before, $\alpha$ may not have a unique geodesic representative; different geodesic representatives of $\alpha$ are parallel and foliate a flat cylinder that we refer to as $F_{\alpha}$. Denote the two boundary curves of $F_{\alpha}$ by $\alpha_{E}$ and $\alpha_{G}$. When $F_{\alpha}$ is degenerate, $\alpha_{E}=\alpha_{G}$.

We say an annulus is regular if its boundary curves are equidistant. Let $E_{\alpha}$ be the largest embedded regular annulus with boundary curve $\alpha_{E}$ and let $G_{\alpha}$ be the largest embedded regular annulus with boundary curve $\alpha_{G}$. Note that $E_{\alpha}$ and $G_{\alpha}$ may intersect $F_{\alpha}$ and each other. In a degenerate case, the interior of some or all of these annuli could be empty, for example, the interior of $F_{\alpha}$ is empty when $\alpha$ has a unique geodesic representative.

We call $\alpha_{E}$, the shared boundary of $E_{\alpha}$ and $F_{\alpha}$, the inner boundary of $E_{\alpha}$ (and similarly $\alpha_{G}$ is the inner boundary of $G_{\alpha}$ ). The annuli $E_{\alpha}$ and $G_{\alpha}$ are 
called expanding because the equidistance curves parallel to the inner boundary get longer as they span $E_{\alpha}$ and $G_{\alpha}$. Let $l=\ell_{q}(\alpha)$ and let $e, f$ and $g$ be the $q$-distances between the boundaries of $E_{\alpha}, F_{\alpha}$ and $G_{\alpha}$ respectively. According to [43], when $\operatorname{Ext}_{X}(\alpha) \leq \epsilon_{0}$, (see Sect. 1.3 for the discussion of the choice of $\epsilon_{0}$ ) we have the following estimates

$$
\frac{1}{\operatorname{Ext}_{X}(\alpha)} \stackrel{*}{`} \operatorname{Mod}\left(E_{\alpha}\right)+\operatorname{Mod}\left(F_{\alpha}\right)+\operatorname{Mod}\left(G_{\alpha}\right)
$$

where

$$
\operatorname{Mod}\left(E_{\alpha}\right) \stackrel{*}{`} \log \frac{e}{l} \quad \operatorname{Mod}\left(F_{\alpha}\right)=\frac{f}{l}, \quad \text { and } \quad \operatorname{Mod}\left(G_{\alpha}\right) \stackrel{*}{`} \log \frac{g}{l} .
$$

Here $\log (\cdot)$ is a modified $\log$ arithm function:

$$
\log (t)=\max \{\log (t), 1\} .
$$

We intend Log to apply only to large numbers. Of course, the value of either $e, f$ or $g$ could be zero and the second line will be $-\infty$. We use the modified logarithm to avoid this issue.

Note that, a simple closed curve that has a short flat length may not have a small extremal length. We need to measure what is the largest neighborhood of $\alpha$ that still has a simple topology. Later, we use this idea to define a notion of extremal length for a saddle connection.

\subsection{Short simple closed curves}

As in Sect. 2.6, we say a curve $\alpha$ is short in $q$ if $\operatorname{Ext}_{X}(\alpha) \leq \epsilon_{0}$. Denote the set of short curves in $q$ by $\mathcal{S}_{q}$. We say $\alpha$ is a cylinder curve if the interior of $F_{\alpha}$ is not empty. In what follows, the cases when $\alpha \in \mathcal{S}_{q}$ is a cylinder curve and $F_{\alpha}$ has a large enough modulus will need special treatments. When the modulus of $F_{\alpha}$ is extremely small, $\alpha$ behaves essentially like a non-cylinder curve. We make this precise:

Definition 3.1 Let $\tau$ be a positive real number and let $\mathrm{M}_{\tau}=e^{-2 \tau}$. We say a curve $\alpha \in \mathcal{S}_{q}$ is a large-cylinder curve if $\operatorname{Mod}\left(F_{\alpha}\right) \geq \mathrm{M}_{\tau}$. Denote the set of large-cylinder curves by $\mathcal{S}_{q}^{\geq \tau}$ and define

$$
\mathcal{S}_{q}^{\leq \tau}=\mathcal{S}_{q} \backslash \mathcal{S}_{q}^{\geq \tau}
$$

For $\alpha \in \mathcal{S}_{q}^{\geq \tau}$, the size $s_{\alpha}$ of $F_{\alpha}$ is defined to be the distance between the boundaries of $F_{\alpha}$. 
Remark 3.2 The constant $\tau$, which is determined in Sect.6, is the distance between steps of a random walk trajectory. We use $M_{\tau}$ instead of just writing $e^{-2 \tau}$ to highlight the fact that $\mathrm{M}_{\tau}$ is a bound for modulus. There is an implicit assumption that $\tau$ is large enough (say, $\tau \geq \tau_{0}$ for some uniform constant $\tau_{0}$ ). That is, unless otherwise stated, all statements hold with uniform constants independent of $\tau$ as long as $\tau \geq \tau_{0}$.

Along Teichmüller geodesics, the length of a curve $\alpha \in \mathcal{S}_{q}^{\leq \tau}$ changes slowly while the modulus of $F_{\alpha}$ remains small. More precisely, let

$$
\left(X_{t}, q_{t}\right)=g_{t}(X, q)
$$

where $g_{t}$ is the Teichmüller geodesic flow. Assuming $\alpha \in \mathcal{S}_{q}^{\leq \tau}$ and $0 \leq t \leq \tau$, we have $\operatorname{Mod}_{q_{t}}\left(F_{\alpha}\right) \stackrel{*}{\prec} 1$. As a consequence of Eqs. (5) and (6), $\operatorname{Mod}_{q_{t}}\left(G_{\alpha}\right)$ and $\operatorname{Mod}_{q_{t}}\left(E_{\alpha}\right)$ change at most linearly and we have

$$
\frac{1}{\operatorname{Ext}_{X}(\alpha)}-t \stackrel{*}{\prec} \frac{1}{\operatorname{Ext}_{X_{t}}(\alpha)} \stackrel{*}{\prec} \frac{1}{\operatorname{Ext}_{X}(\alpha)}+t .
$$

\subsection{The thick-thin decomposition of quadratic differentials}

We call the components of $S \backslash \mathcal{S}_{q}$ the thick subsurfaces of $q$. The homotopy class of each such subsurface $Q$ of $S$ has a representative with $q$-geodesic boundaries. There is, in fact, a unique such representative that is disjoint from the interior of cylinders associated to the boundary curves of $Q$. This can also be described as the smallest representative of $Q$ with $q$-geodesic boundaries. We denote this subsurface by $Q$ as well. Define the size $s_{Q}$ of $Q$ to be the $q$ diameter of this representative. The following theorem states that the geometry of the subsurface $Q$ is essentially the same as that of the thick hyperbolic subsurface of $X$ in the homotopy class of $Q$ but scaled down to a size $s_{Q}$ :

Theorem 3.3 [42] For every essential closed curve $\gamma$ in $Q$,

$$
\ell_{X}(\gamma) \stackrel{*}{`} \sqrt{\operatorname{Ext}_{X}(\gamma)} \stackrel{*}{`} \frac{\ell_{q}(\gamma)}{s_{Q}} .
$$

In particular, the q-length of shortest essential curve in $Q$ is on the order of $s_{Q}$.

Example 3.4 A quadratic differential can be described as a singular flat structure of a surface plus a choice of a vertical direction. For example, the surface obtained from the polygon in Fig. 2 with the given edge identifications is a once punctured genus 2 surface. Assume that the edges 2, 3, 5 and 6 have a 

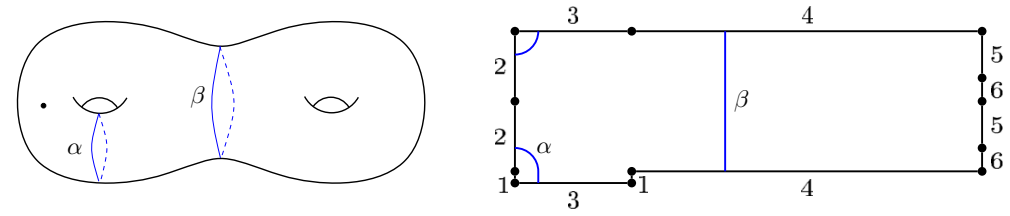

Fig. 2 Quadratic differential $(X, q)$ and short curves of $X$
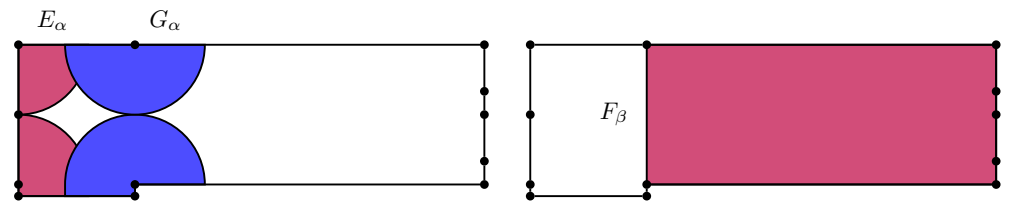

Fig. 3 The maximal expanding annuli $E_{\alpha}$ and $G_{\alpha}$ and the maximal flat annulus $F_{\beta}$

comparable lengths, the edge 1 is significantly shorter and the edge 4 is significantly longer than the others. Choose an arbitrary vertical direction and let $(X, q)$ be the associated quadratic differential.

Then the hyperbolic metric on $X$ has two short simple closed curves; $\mathcal{S}_{q}=$ $\{\alpha, \beta\}$. The curve $\beta$ is a cylinder curve and has a small extremal length because the flat annulus $F_{\beta}$ (Fig. 3) has a large modulus. In fact, $\beta$ is a large-cylinder curve $\left(\mathcal{S}_{q}^{\geq \tau}=\{\beta\}\right)$. The curve $\alpha$ is a non-cylinder curve and it has a small extremal length because the expanding annuli $E_{\alpha}$ and $G_{\alpha}$ (Fig. 3) have large moduli $\left(\mathcal{S}_{q}^{\leq \tau}=\{\alpha\}\right)$. Note that the $q$-geodesic representative of $\alpha$ is the saddle connection 1 (the end points of arc 1 are identified).

There are two thick subsurfaces. There is a once punctured torus with a boundary curve $\beta$ whose $q$-representative is degenerate and is represented in $q$ with a graph of area zero (the union of arcs 5 and 6). The other is a pair of pants whose boundaries consist of two copies of $\alpha$ and one copy of $\beta$. The maximal expanding annuli $E_{\alpha}$ and $G_{\alpha}$ do not necessarily stay inside of the $q$-representative of this pair of pants and they may overlap.

The size of a thick subsurface $Q$ is related to the radii of annuli $E_{\alpha}, F_{\alpha}$ and $G_{\alpha}$ for every boundary curve $\alpha$. We make a few observations that will be useful later.

Lemma 3.5 Let $Q$ be a thick subsurface of $(X, q), \alpha$ be a boundary component of $Q$ and $E_{\alpha}$ be the expanding annulus in the direction of $Q$. Using the notation of Eq. (5) we have

1. $l \leq 2 s_{Q}$.

2. $e \leq s_{Q}$.

3. $\max (e, f, g) \geq \ell_{q}(\alpha)$.

4. $(e+l) \stackrel{*}{`} s_{Q}$.

5. If $\operatorname{Mod}\left(E_{\alpha}\right) \stackrel{*}{\succ} 1$ then $e^{*} s_{Q}$. 
Proof Since $\alpha$ is part of $Q$, its length is less than twice the diameter of $Q$ which is the first assertion. To see part two, note that if $e$ is larger than $s_{Q}$, then $Q$ is contained in $E_{\alpha}$ which is an annulus. This is a contradiction. Part (3) follows from Eq. (6) and the fact that $\alpha$ is $\epsilon_{0}$-short. Parts (1) and (2) imply $(e+l) \stackrel{*}{\prec} s_{Q}$. Hence, to prove part (4), we need to show $(e+l) \stackrel{*}{\succ} s_{Q}$.

Since $E_{\alpha}$ is maximal, its outer boundary self-intersects. Let $\gamma$ be the curve constructed as a concatenation a sub arc of $\alpha$ and two arcs connecting $\alpha$ to the boundary points of $E_{\alpha}$ associated to the self intersection of $E_{\alpha}$. Note that the inner boundary of $E_{\alpha}$ is a geodesic and its outer boundary has positive curvature, therefore, the interior of $E_{\alpha}$ is convex, and the curve $\gamma$ must be essential.

Then $l+e \succ^{*} \ell_{q}(\gamma)$. If $\gamma$ is contained in $Q$ and is essential in $Q$, then $\operatorname{Ext}_{X}(\gamma) \stackrel{*}{\succ} 1$ ( $Q$ is a thick subsurface). From Theorem 3.3 we get,

$$
\frac{\ell_{q}(\gamma)}{s_{Q}} \stackrel{*}{\succ} 1 \text { and hence }(e+l) \stackrel{*}{\succ} s_{Q}
$$

If $\gamma$ is not contained in $Q$, we show that there exists a closed curve $\gamma^{\prime}$ in $Q$ whose length is not much longer than $\gamma$.

Assume that $\gamma$ exists $Q$ by intersecting a boundary curve $\alpha^{\prime}$ and returns via a boundary curve $\alpha^{\prime \prime}$ ( $\alpha^{\prime}$ and $\alpha^{\prime \prime}$ maybe the same curve). By part (3), $\max \left(e^{\prime}, f^{\prime}, g^{\prime}\right)$ is larger than $l^{\prime}, \max \left(e^{\prime \prime}, f^{\prime \prime}, g^{\prime \prime}\right)$ is larger than $l^{\prime \prime}$ and $\ell_{q}(\gamma)$ is larger than both. There is a sub-arc $\omega$ of $\gamma$ connecting $\alpha^{\prime}$ to $\alpha^{\prime \prime}$, in particular, $\ell_{q}(\omega) \leq \ell_{q}(\gamma)$. If $\alpha^{\prime} \neq \alpha^{\prime \prime}$, let $\gamma^{\prime}$ be the curve obtained as a concatenation of two copies of $\omega$ and a copy of $\alpha^{\prime}$ and $\alpha^{\prime \prime}$ each. This curve is essential in $Q$ unless $Q$ is a pair of pants, in which case, we take $\gamma^{\prime}$ to be the curve that wraps around $\alpha^{\prime}$ twice. If $\alpha^{\prime}=\alpha^{\prime \prime}$, then let $\gamma^{\prime}$ be the curve obtained as a concatenation of $\omega$ and a sub-arc of $\alpha^{\prime}$. Again, this curve is essential in $Q$ unless $Q$ is a pair of pants, in which case, we take $\gamma^{\prime}$ to be the curve that wraps around $\alpha^{\prime}$ twice. The curve $\gamma^{\prime}$ resides in $Q$ and $\ell_{q}\left(\gamma^{\prime}\right) \stackrel{*}{\prec} \ell_{q}(\gamma)$. We have

$$
(e+l) \stackrel{*}{\succ} \ell_{q}(\gamma) \stackrel{*}{\succ} \ell_{q}\left(\gamma^{\prime}\right) \stackrel{*}{\succ} s_{Q} .
$$

To see part (5), we note that, if

$$
\log \frac{e}{l} \stackrel{*}{`} \operatorname{Mod}\left(E_{\alpha}\right) \stackrel{*}{\succ} 1 \text { then } e \stackrel{*}{`}(e+l) .
$$

Now, part (5) follows from part (4).

As a corollary we get the following analogue of the collar lemma: 
Corollary 3.6 Let $\alpha \in \mathcal{S}_{q}$ be the boundary of a thick subsurface $Q$ and let $\gamma$ be any curve crossing $\alpha$. Then

$$
\ell_{q}(\gamma) \stackrel{*}{\succ} s_{Q}
$$

Proof We have $\ell_{q}(\gamma) \geq \max (e, f, g)$ and by part (3) of Lemma 3.5, $\max (e, f, g) \geq l$. Hence, $\ell_{q}(\gamma) \stackrel{*}{\succ}(e+l)$. The corollary now follows from part (4) of Lemma 3.5.

\subsection{Extremal lengths and flat lengths of saddle connections}

As mentioned above, we can also define a notion of extremal length for saddle connections. Let $\omega$ be a saddle connection connecting two distinct critical points in $(X, q)$. Let $E_{\omega}$ be the annulus obtained by taking the largest regular neighborhood of $\omega$ that is still a topological disk and then cutting a slit open along $\omega$. Let $l=\ell_{q}(\omega)$ and $e$ be the radius of $E_{\omega}$ (the $q$-distance between $\omega$ and the boundary of $E_{\omega}$ ). Then, we define [the second inequality follows from Eq. (6)]

$$
\operatorname{Ext}_{q}(\omega):=\frac{1}{\log (e / l)} \stackrel{*}{`} \frac{1}{\operatorname{Mod}\left(E_{\omega}\right)} .
$$

Another interpretation of this notion of extremal length, that would provide roughly the same result, is to compute the extremal length in a ramified double cover of $(X, q)$. Denote the end points of $\omega$ by $p_{1}$ to $p_{2}$. There exists a unique ramified double cover $\phi: X_{\omega} \rightarrow X$ with simple ramification points at only $p_{1}$ and $p_{2}$. Note that $\alpha_{\omega}=\phi^{-1} \omega$ is a simple closed curve on $X_{\omega}$.

Lemma 3.7 If $\operatorname{Ext}_{q}(\omega) \leq \epsilon_{0}$, then

$$
\operatorname{Ext}_{X_{\omega}}\left(\alpha_{\omega}\right) \stackrel{*}{`} \operatorname{Ext}_{q}(\omega)
$$

Proof Let $q_{\omega}$ be the lift of $q$ to $X_{\omega}$. Note that $\alpha_{\omega}$ has a unique geodesic representative in $q_{\omega}\left(\operatorname{Mod}\left(F_{\alpha_{\omega}}\right)=0\right)$ and $E_{\alpha_{\omega}}$ and $G_{\alpha_{\omega}}$ are conformally equivalent to $E_{\omega}$. Hence, by Eq. (5)

$$
\frac{1}{\operatorname{Ext}_{X_{\omega}}\left(\alpha_{\omega}\right)} \stackrel{*}{`} \operatorname{Mod}\left(E_{\alpha_{\omega}}\right)+\operatorname{Mod}\left(G_{\alpha_{\omega}}\right)=2 \operatorname{Mod}\left(E_{\omega}\right) \stackrel{*}{ } \frac{1}{\operatorname{Ext}_{q}(\omega)} .
$$


Since $l$ and $e$ change at most exponentially fast along a Teichmüller geodesic, similar to Eq. (7), for $q_{t}=g_{t}(q)$ we have

$$
\frac{1}{\operatorname{Ext}_{q}(\omega)}-t \stackrel{*}{\prec} \frac{1}{\operatorname{Ext}_{q_{t}}(\omega)} \stackrel{*}{\prec} \frac{1}{\operatorname{Ext}_{q}(\omega)}+t .
$$

Definition 3.8 For any $0<\epsilon \leq \epsilon_{0}$, let $\Omega_{q}(\epsilon)$ be the set of saddle connections $\omega$ of $q$ so that, either

- $\operatorname{Ext}_{q}(\omega) \leq \epsilon$, or

- $\omega$ lies on a geodesic representative for $\alpha$ with $\operatorname{Ext}_{X}(\alpha) \leq \epsilon$.

Later in the text, we will add further restrictions on the value of $\epsilon$ depending on $\tau$ (see Lemma 3.13 and Lemma 6.1). We note however that, in all the proofs, making $\epsilon$ smaller or making $\tau$ larger does not effect the constants involved in any of our estimates.

In general, knowing $\ell_{q}(\omega)$ is small does not imply that $\omega$ has a small extremal length. However, we have the following lemma which is enough to show that Theorem 1.5 follows from Theorem 1.5.

Lemma 3.9 Assume that $(X, q)$ has a saddle connection $\omega$ with $\ell_{q}(\omega) \ll 1$. Then, either

$$
\frac{1}{\operatorname{Ext}_{q}(\omega)} \stackrel{*}{`} \log \frac{1}{\ell_{q}(\omega)} \text { or } \frac{1}{\operatorname{Ext}_{X}(\alpha)} \stackrel{*}{\succ} \log \frac{1}{\ell_{q}(\omega)},
$$

for some simple closed curve $\alpha$. In particular, $\Omega_{q}(\epsilon)$ is non-empty.

Proof Let $l=\ell_{q}(\omega)$ and $e$ be the radius of $E_{\omega}$. Since the boundary of $E_{\omega}$ self intersects ( $E_{\omega}$ is maximal), there is a simple closed curve $\gamma$, obtained by a concatenation of a sub arc of $\omega$ and two arcs connecting $\omega$ to the boundary of $E_{\omega}$, with $\ell_{q}(\gamma) \stackrel{*}{\prec}(e+l)$.

Assume first that $\mathcal{S}_{q}$ is empty. Then, $\ell_{q}(\gamma) \stackrel{*}{\succ} 1$. Since, $e \stackrel{*}{\prec} 1$, we have

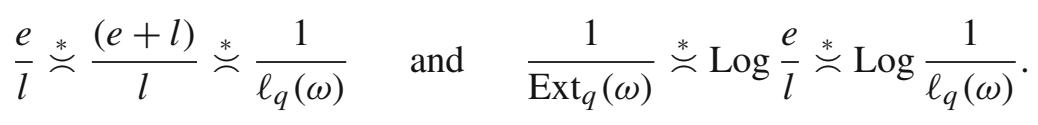

That is, the first inequality holds. Otherwise, we show that, there is a curve $\alpha_{1} \in \mathcal{S}_{q}$ with $\ell_{q}\left(\alpha_{1}\right) \stackrel{*}{\prec}(e+l)$. This is because, either $\gamma \in \mathcal{S}_{q}$ and we can take $\alpha_{1}=\gamma$ or $\gamma$ intersects a thick subsurface $Q$ in which case we let $\alpha_{1}$ be any boundary component of $Q$. Using Corollary 3.6 and part one of Lemma 3.5, we get:

$$
(e+l) \stackrel{*}{\succ} \ell_{q}(\gamma) \stackrel{*}{\succ} s_{Q} \stackrel{*}{\succ} \ell_{q}\left(\alpha_{1}\right) .
$$


Since the total area of $q$ is 1 , there is always a thick subsurface of size comparable to 1 . Let $Q_{1}, \ldots, Q_{k}$ be a sequence of distinct subsurfaces of sizes $s_{1}, \ldots, s_{k}$ respectively, where $\alpha_{1}$ is a boundary component of $Q_{1}, Q_{i-1}$

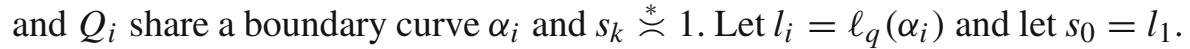

Consider $G_{\alpha_{i}}$, the expanding annulus with inner boundary $\alpha_{i}$ in the direction of $Q_{i}$ with radius $g_{i}$. For $i \geq 1$, part (4) of Lemma 3.5 implies, $\left(g_{i}+l_{i}\right) \stackrel{*}{`} s_{i}$ and by part (1) $s_{i-1} \stackrel{*}{\succ} l_{i}$. Hence, from Eq. (5), we know that

$$
\frac{1}{\operatorname{Ext}_{q}\left(\alpha_{i}\right)} \stackrel{*}{\succ} \max \left(\log \frac{g_{i}}{l_{i}}, 1\right) \stackrel{+}{\succ} \log \frac{g_{i}+l_{i}}{l_{i}} \succ \log \frac{s_{i}}{s_{i-1}} .
$$

That is, the common boundary curve of two surfaces of very different size has a very small extremal length. Also, (recall that $\left.s_{0}=l_{1} \stackrel{*}{\prec}(e+l)\right)$ :

$$
\left(\prod_{i=1}^{k} \frac{s_{i}}{s_{i-1}}\right) \frac{e+l}{l} \asymp\left(\frac{1}{s_{0}}\right) \frac{e+l}{l} \stackrel{*}{\succ} .
$$

Here, the maximum value of $k$ depends only on the topology of $S$. Therefore, taking the logarithm of both sides of Eq. (9), we conclude that either

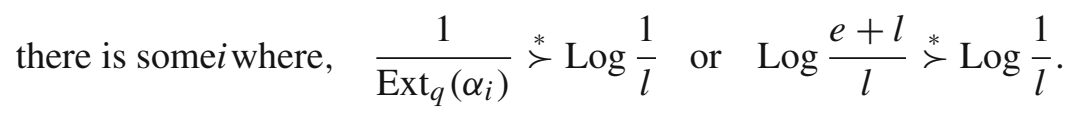

In the first case, the lemma holds for $\alpha=\alpha_{i}$. In second case,

$$
\frac{1}{\operatorname{Ext}_{q}(\omega)} \stackrel{*}{*} \log \frac{e}{l} \stackrel{*}{*} \log \frac{e+l}{l} \stackrel{*}{`} \log \frac{1}{\ell_{q}(\omega)} .
$$

Remark 3.10 Note that in both Lemma 3.7 and Lemma 3.9 the implied constants only depend on the topology of $S$.

\subsection{A $(q, \tau)$-regular triangulation}

We would like to mark a quadratic differential $q$ by a triangulation where the edges have a bounded length. However, the notion of having a bounded length should depend on which thick subsurface we are in. That is, we would like the $q$-length of an edge to not be longer than the size of the thick subsurfaces it intersects. The complication comes from the fact that a saddle connection may intersect several thick subsurfaces of various sizes.

Also, as mentioned before, large-cylinder curves will require a special treatment. Hence, we triangulate only the complement of large-cylinders. Recall 
that two saddle connections are said to be disjoint if they have disjoint interiors but they may share one or two end points.

Definition 3.11 Let $(X, q)$ be a quadratic differential. Given a cylinder curve $\alpha$, let $v_{\alpha}$ be an arc connecting the boundaries of $F_{\alpha}$ that is perpendicular to $\alpha$. By a $(q, \tau)$-regular triangulation $T$ of $q$ we mean a collection of disjoint saddle connections satisfying the following conditions:

1. For $\alpha \in \mathcal{S}_{q}^{\geq \tau}$, denote the interior of a cylinder $F_{\alpha}$ by $F_{\alpha}^{\circ}$. Then, $T$ is disjoint from $F_{\alpha}^{\circ}$ and it triangulates their complement

$$
q \backslash \bigcup_{\alpha \in \mathcal{S}_{q}^{\geq \tau}} F_{\alpha}^{\circ} .
$$

That is, the complement of $T$ is a union of triangles and large-cylinders $F_{\alpha}^{\circ}, \alpha \in \mathcal{S}_{q}^{\geq \tau}$. In particular, $T$ contains the boundaries of $F_{\alpha}$.

2. If an edge $\omega$ of $T$ intersects a thick subsurface $Q$ of $q$ then $\ell_{q}(\omega) \stackrel{*}{\prec} s_{Q}$.

3. If $\alpha$ is a cylinder curve in $\mathcal{S}_{q}^{\leq \tau}$ then $v_{\alpha}$ intersects $T$ a uniformly bounded number of times.

We shall see that condition 3 means that the triangulation $T$ does not twist around short simple closed curves.

Remark 3.12 It is important to choose the implied constants in conditions 2 and 3 in Definition 3.11 large enough so that every quadratic differential $q$ has a $(q, \tau)$-regular triangulation. In fact, we choose the constants so that the key Lemma 3.13 below holds.

Lemma 3.13 For every $\tau$ there is $\epsilon_{1}(\tau)$ so that for $\epsilon<\epsilon_{1}(\tau)$ the following holds. Let $\Omega$ be a subset of $\Omega_{q}(\epsilon)$ consisting of pairwise disjoint saddle connections. Then $\Omega$ can be extended to a $(q, \tau)$-regular triangulation $T$.

Proof We would like to triangulate each thick piece Q separately and let $T$ be the union of these triangulations. However, saddle connections in $\Omega$ may intersect a boundary curve $\alpha$ of $Q$. To remedy this, we perturb $\alpha$ slightly to a curve $\bar{\alpha}$ that is a union of saddle connections, lies in a small neighborhood of $\alpha$ and is disjoint from $\Omega$ (see Claim 1). These curves divide the surface into subsurfaces with nearly geodesic boundaries. We denote the surface associated to $Q$ with $\bar{Q}$. We then extend $\Omega$ to a triangulation in each $\bar{Q}$ so that the edge lengths are not much longer than the diameter of $\bar{Q}$ which is comparable to $s_{Q}$ (see Claim 3) and let $T$ be the union of these triangulations. However, one needs to be careful that $\bar{Q}$ does not intersect any subsurface of size much smaller that $s_{Q}$, otherwise the resulting triangulation would not be $(q, \tau)$-regular. 
Claim 1 For every $\alpha \in \mathcal{S}_{q}$, there is a representative $\bar{\alpha}$ of $\alpha$ that is a union of saddle connections, lies in a $\left(\ell_{q}(\alpha) / 2\right)$-neighborhood of $\alpha$ and is disjoint from $\Omega$. For $\alpha, \beta \in \mathcal{S}_{q}, \bar{\alpha}$ and $\bar{\beta}$ do not intersect. Furthermore, if $\alpha$ is a boundary of $Q$ then $\bar{\alpha}$ intersects only surfaces that are larger than $Q$, namely, if $\bar{\alpha}$ intersects a thick subsurface $Q^{\prime}$ we have:

$$
s_{Q^{\prime}} \stackrel{*}{\succ} s_{Q}
$$

Proof of Claim 1: Let $\alpha \in \mathcal{S}_{q}$ be a common boundary of thick subsurfaces $Q$ and $R$. Recall that $\mathrm{M}_{\tau}=2^{-2 \tau}$. If $\operatorname{Mod}\left(F_{\alpha}\right) \geq \mathrm{M}_{\tau}$, we can choose $\epsilon_{1}$ small enough to ensure that $\alpha$ is disjoint from $\Omega$. This is because, if $\omega$ is part of a short curve $\alpha^{\prime}$, then $\omega$ is disjoint from $\alpha$ because short curves $\alpha$ and $\alpha^{\prime}$ do not intersect. Otherwise, $\omega$ has to satisfy the first assumption in Definition 3.8. But, $F_{\alpha}$ does not contain any singular points and any arc $\omega \in \Omega$ intersecting $\alpha$ has to cross $F_{\alpha}$. Therefore, $\ell_{q}(\omega) \geq f_{\alpha}\left(f_{\alpha}\right.$ is the distance between the boundaries of $F_{\alpha}$ ) and, for the radius $e_{\omega}$ of $E_{\omega}$, we have $e_{\omega} \leq \ell_{q}(\alpha)$ (otherwise $\alpha$ would be contained in $\left.E_{\omega}\right)$. But $\operatorname{Mod}\left(F_{\alpha}\right)=\frac{f_{\alpha}}{\ell_{q}(\alpha)} \geq \mathrm{M}_{\tau}$ and thus (the second inequality follows from Eq. (6))

$$
\frac{1}{\epsilon_{1}} \leq \frac{1}{\operatorname{Ext}_{q}(\omega)} \stackrel{*}{`} \log \frac{e_{\omega}}{\ell_{q}(\omega)} \leq \log \frac{\ell_{q}(\alpha)}{f_{\alpha}} \leq \log \frac{1}{\mathrm{M}_{\tau}}=2 \tau .
$$

which is not possible if $\epsilon_{1}$ is chosen to be small enough. To summarize, if $\operatorname{Mod}\left(F_{\alpha}\right) \geq \mathrm{M}_{\tau}$, then $\alpha$ is already disjoint from $\Omega$, we can take $\bar{\alpha}=\alpha$.

If $\operatorname{Mod}\left(F_{\alpha}\right) \leq \mathrm{M}_{\tau}$, then either $E_{\alpha}$ or $G_{\alpha}$ has a large modulus. The annulus with the larger modulus is in the direction of the thick surface with the larger size (Lemma 3.5). Assume $E_{\alpha}$, the annulus in the direction of $Q$, has a large modulus. Let $e_{\alpha}$ be the distance between the boundaries of $E_{\alpha}$. By part (5) of Lemma 3.5 and the previous assumption we have

$$
e_{\alpha} \stackrel{*}{`} s_{Q} \geq s_{R}
$$

Denote the $\left(\ell_{q}(\alpha) / 2\right)$-neighborhood of $\alpha$ in $E_{\alpha}$ with $\bar{E}_{\alpha}$. The annulus $E_{\alpha}$ may not be contained entirely in $Q$ and may intersect some thick subsurfaces with very small size. But $\bar{E}_{\alpha}$ does not intersect any small subsurfaces. To see this, assume $Q^{\prime}$ intersects $\bar{E}_{\alpha}$. Since $Q^{\prime}$ is disjoint from $\alpha$, it has to enter $E_{\alpha}$ intersecting the outer boundary of $E_{\alpha}$. But $e_{\alpha}$ is much larger than $\ell_{q}(\alpha)$, and hence:

$$
s_{Q^{\prime}} \stackrel{*}{\succ} \ell_{q}\left(\partial Q^{\prime}\right)>e-\ell_{q}(\alpha) / 2 \stackrel{*}{\succ} s_{Q} .
$$

Thus, the last condition of the claim is satisfied as long as $\bar{\alpha}$ stays in $\bar{E}_{\alpha}$. 
Note that no arc in $\Omega$ can cross $\bar{E}_{\alpha}$ (intersect both boundaries). This is because, if $\omega$ is an arc in a curve $\beta \in \mathcal{S}_{q}$, then it does not intersect $\alpha$ since $\beta$ and $\alpha$ have intersection number zero. Otherwise, $\operatorname{Ext}_{q}(\omega)$ is small, which implies that its length is much less than the injectivity radius of any point along $\omega$. But the injectivity radius of any point in $\bar{E}_{\alpha}$ is less that $2 \ell_{q}(\alpha)$. Hence, (by choosing $\epsilon$ small enough) $\ell_{q}(\omega)$ is less than the distance between the boundaries of $\bar{E}_{\alpha}$ with is equal to $\ell_{q}(\alpha) / 2$.

Consider the union of $\alpha$ and the set $\Omega_{\alpha}$ of arcs in $\Omega$ that intersect $\alpha$. The convex hull $H_{\alpha}$ of this set in $\bar{E}_{\alpha}$ is an annulus (perhaps degenerate). We observe that the interior of $H_{\alpha}$ does not contain any singular points. Otherwise, there would be a geodesic quadrilateral, where two edges are subsegments of arcs in $\Omega_{\alpha}$ and one edge is a subsegment of $\alpha$, that contains a singular point in its interior. But this violates the Gauss-Bonnet theorem. Let $\bar{\alpha}$ be the boundary component of $H_{\alpha}$ that is not $\alpha$. Then $\bar{\alpha}$ is in the homotopy class of $\alpha$ and lies inside $\bar{E}_{\alpha}$. Also, because the interior $H_{\alpha}$ does not contain any singular points, $\bar{\alpha}$ is disjoint from every saddle connection in $\Omega$. Furthermore, by the triangle inequality, any saddle connection $\bar{\omega}$ that appears in $\bar{\alpha}$ has a $q$-length less than or equal to $2 \ell_{q}(\alpha)$.

It remains to show that for $\alpha, \beta \in \mathcal{S}_{q}, \bar{\alpha}$ and $\bar{\beta}$ are disjoint. Assume $\ell_{q}(\beta) \geq \ell_{q}(\alpha)$. Then, $\alpha$ is disjoint from $\bar{E}_{\beta}$, otherwise, $\alpha$ would be contained in $E_{\beta}$ which is an annulus an does not contain any curve non-homotopic to $\beta$. This means $\alpha$ is disjoint from $\bar{\beta}$ which is contained in $\bar{E}_{\beta}$. Also, since $H_{\beta}$ contains no singular points, if a saddle connection $\omega \in \Omega_{\alpha}$ intersects $\bar{\beta}$ then it also intersects $\beta$. But then $\omega$ is in $\Omega_{\beta}$ and hence it is disjoint from $\bar{\beta}$. Therefore, $\bar{\beta}$ is disjoint from the convex hull $H_{\alpha}$ and thus also from $\bar{\alpha}$. This finishes the proof of claim 1 .

Next, let $\bar{\Omega}$ be the set of edges that appear in curves $\bar{\alpha}$ for every $\alpha \in \mathcal{S}$. We have shown that saddle connections in $\bar{\Omega}$ are disjoint from those in $\Omega$. After removing the interiors of large cylinders from the quadratic differential $(X, q)$ and cutting along curves $\bar{\alpha}, \alpha \in \mathcal{S}_{q}$, we obtains a collection of subsurfaces with nearly geodesic boundaries. Denote the representative of a thick subsurface $Q$ that is disjoint from curves $\bar{\alpha}$ by $\bar{Q}$.

For each $\alpha \in \mathcal{S}_{q}^{\leq \tau}$, if $F_{\alpha}^{\circ}$ is disjoint from every saddle connection in $\Omega \cup \bar{\Omega}$, we choose a saddle connection $\omega_{\alpha}$ that crosses $F_{\alpha}$, is disjoint from $v_{\alpha}$ (does not twists around $\alpha$ ). In particular, $\omega_{\alpha}$ is disjoint from every saddle connection $\Omega \cup \bar{\Omega}$ and has a length that is comparable with $\ell_{q}(\alpha)$. Let $\Omega^{n}$ denote the set of such saddle connections $\omega_{\alpha}$.

Claim 2 Saddle connections in

$$
T_{0}=\Omega \cup \bar{\Omega} \cup \Omega^{n}
$$

satisfying conditions (2-3) of Definition 3.11. 
Proof of Claim 2: All the conditions follow immediately from the construction, but the argument is long since we have to look at all the cases. We have already shown that these edges satisfy condition (1) and arcs in $\bar{\alpha}$ satisfy condition (2). To see that an arc $\omega \in \Omega$ satisfies condition (2) note that if it did not, $\omega$ would intersect a thick subsurface $Q$ with $\ell_{q}(\omega) \geq s_{Q}$. The radius of $E_{\omega}$ is much larger than length of $\omega\left(\log \frac{e_{\omega}}{l_{\omega}} \geq \frac{1}{\epsilon_{1}}\right)$, which implies $E_{\omega}$ contains $Q$. This is a contradiction.

We show that arcs in $T_{0}$ satisfy condition (3). Namely, if $\omega \in \Omega$ intersects a cylinder $F_{\alpha}$, we need to show that $\omega$ intersects $v_{\alpha}$ a bounded number of times. In fact, if they intersect more than once, then $\ell_{q}(\omega) \geq \ell_{q}(\alpha)$. But then $E_{\omega}$ would contain the curve $\alpha$ which is a contradiction ( $E_{\omega}$ is a topological disk). Also, the curve $\bar{\alpha}$ is a convex hull of the union of the curve $\alpha$ which is disjoint from $F_{\alpha}$ and a bounded number of arcs in $\Omega$, each of which intersect $v_{\alpha}$ at most once. Hence $\bar{\alpha}$ intersects $v_{\alpha}$ at most a bounded number of times and thus $\operatorname{arcs}$ in $\bar{\alpha}$ satisfy condition (3).

Since, for every $\alpha \in \mathcal{S}_{q}^{\leq \tau}$, there is a saddle connection in $T_{0}$ crossing $F_{\alpha}$, any triangulation containing $T_{0}$ is guaranteed to satisfy the condition (3).

In the next claim, we describe how to add the remaining edges to $T_{0}$ while still satisfying conditions (1) and (2).

Claim 3 A partial triangulation of $\bar{Q}$ where the length of edges are less than a fixed multiple of $s_{Q}$ can be extended to a triangulation using saddle connections of length less than a larger fixed multiple of $s_{Q}$.

Proof of claim 3: We prove the claim by induction. Start by cutting $\bar{Q}$ along the given edges. Each cutting increases the diameter by at most twice the length of edge being cut. Hence, in the end, we have several components each with diameter comparable to $s_{Q}$. If all components are triangles, we are done. Otherwise, some component contains a saddle connection that is not part of its boundaries or the given triangulation, the shortest such saddle connection has a length less than the diameter of the component it is in, which is comparable to $s_{Q}$ (again, see [49, Proposition 3.1]). The claim follows from the fact that this process ends after a uniformly bounded number of times. The diameter grows at most multiplicatively each time but still it is uniformly bounded multiple of $s_{Q}$ in the end. We choose the constant in the second condition of a $(q, \tau)$ regular triangulation large enough so that the outcome of this algorithm is in fact a $(q, \tau)$-regular triangulation.

The triangulation $T$ is now defined to be the union of all the saddle connections in $T_{0}$ and those coming from claim 3. The newly added edges in $\bar{Q}$ have a $q$-length less than a fixed multiple of $s_{Q}$ and, for any thick subsurface $R$ that $\bar{Q}$ intersects, we have $s_{Q} \stackrel{*}{\prec} s_{R}$. Hence, the condition (2) in Definition 3.11 is satisfied. Therefore, the resulting triangulation $T$ is $(q, \tau)$-regular. 


\subsection{Twisting and extremal lengths}

In this section we define several notions of twisting and discuss how they relate to each other. This is essentially the definition introduced by Minsky extended to a slightly more general setting. We denote the relative twisting of two objects or structures around a curve $\alpha$ by twist ${ }_{\alpha}(\cdot, \cdot)$. This is often only coarsely defined, that is, the value of $\operatorname{twist}_{\alpha}(\cdot, \cdot)$ is determined up to a uniformly bounded additive error.

In the simplest case, let $A$ be an annulus with core curve $\alpha$ and let $\beta$ and $\gamma$ be homotopy classes of arcs connecting the boundaries of $A$ (here, homotopy is relative to the end points of an arc). The relative twisting of $\beta$ and $\gamma$ around $\alpha$, twist $\alpha(\beta, \gamma)$, is defined to be the geometric intersection number between $\beta$ and $\gamma$.

Now consider a more general case where $\alpha$ is a curve on the surface $S$ and $\beta$ and $\gamma$ are two transverse curves to $\alpha$. Let $\tilde{S}_{\alpha}$ be the annular cover of $S$ associated to $\alpha$ and denote the core curve of $\tilde{S}_{\alpha}$ again by $\alpha$. Let $\tilde{\beta}$ and $\tilde{\gamma}$ be the lifts of $\beta$ and $\gamma$ to $\tilde{S}_{\alpha}$ (respectively) that connect the boundaries of $\tilde{S}_{\alpha}$. Note that freely homotopic curves lift to arcs that are homotopic relative their endpoints. The $\operatorname{arc} \tilde{\beta}$ is not uniquely defined, however any pair of lifts are disjoint. We now define

$$
\operatorname{twist}_{\alpha}(\beta, \gamma)=\operatorname{twist}_{\alpha}(\tilde{\beta}, \tilde{\gamma}),
$$

using the previous case. This is well defined up to an additive error of 2 (see [36]).

We can generalize this further and define twisting between any two structures on $S$ as long as the structures in question provide a (nearly) canonical choice of a homotopy class of an $\operatorname{arc} \tilde{\beta}$ connecting the boundaries of $\tilde{S}_{\alpha}$. Then we say the given structure defines a notion of zero twisting around $\alpha$. The relative twisting between two structures is the relative twisting between the associated $\operatorname{arcs}$ in $\tilde{S}_{\alpha}$. Here are a few examples:

- Let $X$ be a Riemann surface. Then $\tilde{\beta}$ can be taken to be the geodesic in $\tilde{X}_{\alpha}$ that is perpendicular to $\alpha$ in the Poincare metric of $\tilde{X}_{\alpha}$. Alternatively, we can pick a shortest curve $\beta$ transverse to $\alpha$ and let $\tilde{\beta}$ be the lift of $\beta$ that connects the boundaries of $\tilde{X}_{\alpha}$. In any case, the choice of $\tilde{\beta}$ is not unique, but any two such transverse arcs have bounded geometric intersection number (see [36]) and the associated relative twisting $\operatorname{twist}_{\alpha}(\cdot, X)$ is well defined up to an additive error.

- Let $q$ be a quadratic differential. As before, $\tilde{\beta}$ can be taken to be the geodesic in $\tilde{q}_{\alpha}$ that is perpendicular to $\alpha$ in the Euclidean metric coming from $q$ or a lift of a $q$-shortest curve $\beta$ transverse to $\alpha$ (see [12]). We denote the associated relative twisting with twist $_{\alpha}(\cdot, q)$. 
- Let $T$ be a $(q, \tau)$-regular triangulation of $(X, q)$ and $\alpha \in \mathcal{S}_{q}^{\leq \tau}$. Then we can choose a curve $\beta$ transverse to $\alpha$ that is carried by $T$ and has a bounded combinatorial length in $T$ and let the lift of $\beta$ to the annular cover of $\alpha$ define zero twisting. Since curves with bounded combinatorial length intersect a bounded number of times, the associated relative twisting $\operatorname{twist}_{\alpha}(\cdot, T)$, is again well defined up to an additive error.

The expression "fix a notion of zero twisting around $\alpha$ " for a curve $\alpha$ in $S$ means "choose a homotopy class of arcs connecting the boundaries of $\tilde{S}_{\alpha}$."

\subsection{Intersection and twisting estimates}

In this section we establish some statements relating Extremal length, twisting and intersection number. We start with a theorem of Minsky giving an estimate for the extremal length of a curve. For a $X \in \mathcal{T}(S)$, let $\mathcal{S}_{X}$ be a set of $\epsilon_{0}$-short simple closed curves in $X$. There is a uniform constant $B$ depending on $\epsilon_{0}$ and the topology of $S$ so that, for every $X$, any curve $\beta$ not in $\mathcal{S}_{X}$ intersects a curve $\gamma$ with $\operatorname{Ext}_{X}(\gamma) \leq B$. That is, the curves with extremal length at most $B$ fill every complementary component of $\mathcal{S}_{X}$. Let $\mathcal{B}_{X}$ be the set of curves with extremal length at most $B$.

Theorem 3.14 (Minsky, [36, Theorem 5.1]) Given $X \in \mathcal{T}(S)$ and a simple closed curve $\gamma \notin \mathcal{S}_{X}$,

$$
\begin{aligned}
\operatorname{Ext}_{X}(\gamma) \stackrel{*}{*} & \max _{\alpha \in \mathcal{S}_{X}} \mathrm{i}(\gamma, \alpha)^{2}\left[\frac{1}{\operatorname{Ext}_{X}(\alpha)}+\operatorname{twist}_{\alpha}^{2}(\gamma, X) \operatorname{Ext}_{X}(\alpha)\right] \\
& +\max _{\alpha \in \mathcal{B}_{X}} \mathrm{i}(\gamma, \alpha)^{2} .
\end{aligned}
$$

The multiplicative constant depends only on the topology of $S$.

It follows from the definition of twisting and elementary hyperbolic geometry that if $\operatorname{twist}_{\alpha}(\beta, X)$ is large (that is, if $\beta$ twists around $\alpha$ a lot), then $\operatorname{Ext}_{X}(\beta) \stackrel{*}{\succ} \operatorname{Ext}_{X}(\alpha)$

Corollary 3.15 For every curve $\gamma$ and any $X \in \mathcal{T}(S)$, there is a curve $\beta$ so that,

$$
\sqrt{\operatorname{Ext}_{X}(\gamma) \operatorname{Ext}_{X}(\beta)} \stackrel{*}{\prec} \mathrm{i}(\gamma, \beta) \quad \text { and } \quad \operatorname{twist}_{\gamma}(X, \beta)=O(1) .
$$

Note that the reverse of first inequality always holds (Eq. (2)).

Proof If $\gamma \in \mathcal{S}_{X}$, then we choose $\beta$ to be a curve that intersects $\gamma$ once or twice, is disjoint from other curves in $\mathcal{S}_{X}$, where twist $\gamma(\beta, X)$ is bounded 
and where $\mathrm{i}(\beta, \alpha)=O(1)$ for $\alpha \in \mathcal{B}_{X}$. Applying Eq. (10) to $\beta$ we have $\operatorname{Ext}_{X}(\beta) \stackrel{*}{`} \frac{1}{\operatorname{Ext}_{X}(\gamma)}$ which implies that the corollary holds for $\beta$ and $\gamma$.

If $\gamma$ is not short in $X$, Theorem 3.14 applies to $\gamma$. Since the number of elements in $\mathcal{S}_{X}$ and $\mathcal{B}_{X}$ is uniformly bounded, $\operatorname{Ext}_{X}(\gamma)$ is comparable to one the following terms:

$$
\frac{\mathrm{i}(\gamma, \alpha)^{2}}{\operatorname{Ext}_{X}(\alpha)}, \quad \mathrm{i}(\gamma, \alpha)^{2} \operatorname{twist}_{\alpha}^{2}(\gamma, X) \operatorname{Ext}_{X}(\alpha) \quad \text { or } \mathrm{i}(\gamma, \alpha)^{2} .
$$

In the fist two cases $\alpha \in \mathcal{S}_{X}$ and in the third case $\alpha \in \mathcal{B}_{X}$. We argue in 3 cases.

If $\operatorname{Ext}_{X}(\gamma) \stackrel{*}{\prec} \frac{\mathrm{i}(\gamma, \alpha)^{2}}{\operatorname{Ext}_{X}(\alpha)}$, for $\alpha \in \mathcal{S}_{X}$, then the corollary holds for $\beta=\alpha$ (the second conclusion follows from the fact that the twisting number of a short curve around a long curve is uniformly bounded).

In the second case, we take $\beta$ to be a curve transverse to $\alpha$ with (see above) $\operatorname{Ext}_{X}(\beta) \stackrel{*}{`} \frac{1}{\operatorname{Ext}_{X}(\alpha)}$ and twist $_{\alpha}(\beta, X)=O(1)$. In particular

$$
\operatorname{twist}_{\alpha}(\gamma, X) \doteq \operatorname{twist}_{\alpha}(\gamma, \beta) \text {. }
$$

The curve $\gamma$ also intersects $\alpha$ and hence $\operatorname{Ext}_{X}(\gamma) \stackrel{*}{\succ} \frac{1}{\operatorname{Ext}_{X}(\alpha)} \stackrel{*}{=} \operatorname{Ext}_{X}(\beta)$. Thus, $\beta$ twist around $\gamma$ at most a uniformly bounded number of times. Also, every strand of $\gamma$ intersecting $\alpha$ intersects $\beta$ at least twist $\alpha(\gamma, \beta)$ times (up to an additive error). In this case twist $\alpha(\gamma, \beta)$ is large and the additive error can be replaced by a multiplicative error to obtain

$$
\mathrm{i}(\gamma, \alpha) \operatorname{twist}_{\alpha}(\gamma, \beta) \stackrel{*}{\prec} \mathrm{i}(\gamma, \beta) .
$$

Therefore,

$$
\begin{aligned}
& \operatorname{Ext}_{X}(\gamma) \stackrel{*}{\prec} \mathrm{i}(\gamma, \alpha)^{2} \operatorname{twist}_{\alpha}^{2}(\gamma, X) \operatorname{Ext}_{X}(\alpha) \quad \text { (Assumption on } \gamma \text { ) } \\
& \stackrel{*}{\prec} \frac{\mathrm{i}(\gamma, \alpha)^{2} \operatorname{twist}_{\alpha}^{2}(\gamma, \beta)}{\operatorname{Ext}_{X}(\beta)} \\
& \stackrel{*}{\prec} \frac{\mathrm{i}(\gamma, \beta)^{2}}{\operatorname{Ext}_{X}(\beta)},
\end{aligned}
$$

which implies the corollary.

The last case is when $\alpha \in \mathcal{B}_{X}$ and $\operatorname{Ext}_{X}(\gamma) \stackrel{*}{\prec} \mathrm{i}(\gamma, \alpha)^{2}$. In this case, we take $\beta=\alpha$. Since $\beta$ has bounded length in $X$,

$$
\text { twist }_{\gamma}(\beta, X)=O(1) \quad \text { and } \quad \operatorname{Ext}_{X}(\beta) \stackrel{*}{`} 1 .
$$


Again, the corollary follows.

We also recall the following lemma ([41, Theorem 4.3]):

Lemma 3.16 (Rafi) For a quadratic differential $(X, q)$ and a Riemann surface $Y \in \mathcal{T}(S)$ with $d_{\mathcal{T}}(X, Y)=O(1)$, we have

$$
\operatorname{twist}_{\alpha}(Y, q) \stackrel{*}{\prec} \frac{1}{\operatorname{Ext}_{X}(\alpha)} .
$$

\subsection{Geometry of quadratic differentials and $(q, \tau)$-regular triangulations}

As we mentioned at the beginning of the section, a $(q, \tau)$-regular triangulation is supposed to capture the geometry of $q$. We make this explicit in the following two lemmas. In Lemma 3.17, we relate the length of a saddle connection to its intersection number with a $(q, \tau)$-regular triangulation. Lemma 3.18 shows that the notion of zero twisting coming from $q$ or $T$ is the same. These are used to prove Lemma 3.19 but more essentially they are needed in Sect. 4.

Lemma 3.17 Let $T$ be a $(q, \tau)$-regular triangulation and $\omega_{T}$ be an edge of $T$. Let $s$ be the minimum of $s_{Q}$ where $Q$ is a thick subsurface of $q$ that intersects $\omega_{T}$. Let $\omega$ be any other saddle connection in $q$ so that, for every curve $\alpha \in \mathcal{S}_{q}^{\leq \tau}$, twist $_{\alpha}(\omega, q)=O(1)$. Then

$$
\mathrm{i}\left(\omega_{T}, \omega\right) \stackrel{*}{\prec} \frac{\ell_{q}(\omega)}{s}+1 .
$$

Proof Condition (2) in the definition of a $(q, \tau)$-regular triangulation implies that $\ell_{q}\left(\omega_{T}\right) \stackrel{*}{\prec} s$. It is sufficient to prove the lemma for a subsegment of $\omega_{T}$ with a $q$-length less than $s / 7$, because $\omega_{T}$ can be covered but uniformly bounded number of such segments. Hence, without loss of generality, we assume $\ell_{q}\left(\omega_{T}\right) \leq s / 7$.

Consider the $s / 7$-neighborhood $N$ of $\omega_{T}$. Then $\omega \cap N$ has at most $O\left(\frac{\ell_{q}(\omega)}{s}\right)$ components. Hence, it is sufficient to show, for every component $\bar{\omega}$ of $\omega \cap N$, that

$$
\mathrm{i}\left(\omega_{T}, \bar{\omega}\right)=O(1) .
$$

First, we claim that any non-trivial curve in $N$ is homotopic to some curve in $\mathcal{S}_{q}$. This is because, any nontrivial loop $\gamma$ in $N$ has a $q$-length of at most $3 s / 7$. By the definition of $s$, it can not be an essential curve in any subsurface $Q$ that $\omega_{T}$ intersects. Assume it intersects curves $\alpha_{1}, \alpha_{2} \in \mathcal{S}_{q}$ that are boundary 


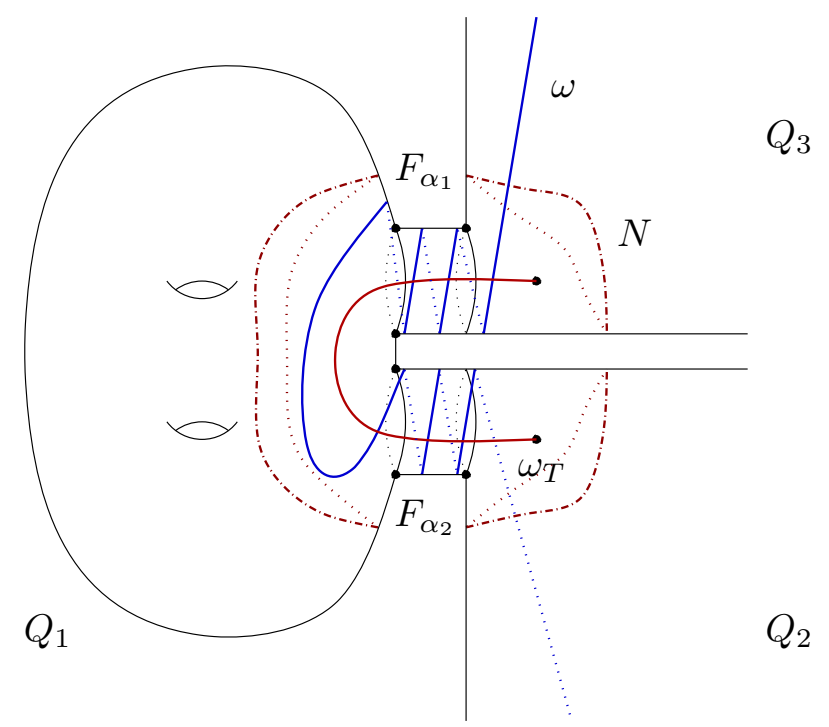

Fig. 4 The $\operatorname{arc} \omega_{T}$ intersects curves $\alpha_{1}, \alpha_{2} \in \mathcal{S}_{q}^{\leq \tau}$ and thick subsurfaces $Q_{1}, Q_{2}$ and $Q_{3}$. Each component $\bar{\omega}$ of $\omega \cap N$ intersects $\omega_{T}$ only a bounded number of times outside of cylinders $F_{\alpha_{1}}$ and $F_{\alpha_{2}}$. The number of intersection points inside of $F_{\alpha_{i}}$ is bounded because of the assumption on the twisting

curves of $Q_{1}\left(\alpha_{1}\right.$ may equal $\left.\alpha_{2}\right)$. Then, $\ell_{q}\left(\alpha_{1}\right)$ and $\ell_{q}\left(\alpha_{1}\right)$ are much smaller than $\ell_{q}(\gamma)$ which is at most $3 s / 7$. But, the sum of $\ell_{q}\left(\alpha_{1}\right), \ell_{q}\left(\alpha_{2}\right)$ and twice the distance between $\alpha_{1}$ and $\alpha_{2}$ (the sum is less than $s$ ) is an upper-bound for the size of $Q$ which is assumed to be larger than $s$. The contradiction proves the claim.

We have shown that a closed curve in $N$ cannon intersect curves in $\mathcal{S}_{q}$. However, the saddle connection $\omega_{T}$ may still intersects some curve $\alpha \in \mathcal{S}_{q}^{\leq \tau}$ (in fact more than one, see Fig. 4). As before, let $v_{\alpha}$ be an arc in $F_{\alpha}$ that connects the boundaries of $F_{\alpha}$ and is perpendicular to them.

First we observe that the number of intersection points between $\omega_{T}$ and $\bar{\omega}$ inside of $F_{\alpha}$ is uniformly bounded. This is because both $\omega_{T}$ and $\bar{\omega}$ intersect $v_{\alpha}$ a uniformly bounded number of times. (This follows from the definition $(q, \tau)$-regular triangulation and the twisting assumption on $\omega$.) If two arcs inside of a cylinder have a large intersection number, at least one of them has to twist around $F_{\alpha}$ a large number of times.

It remains to show that the number of intersection points outside of all cylinders $F_{\alpha}$ is bounded. To see this we observe that, for any thick subsurface $Q$, it is not possible to have a subsegment of $\omega_{T}$ and a subsegment of $\bar{\omega}$ that are contained in $Q$ and have the same endpoint. Otherwise, the concatenation would create a two segment curve $\beta$ that is non-trivial in $N$. Hence, it has to be homotopic to some curve $\alpha \in \mathcal{S}_{q}$. Which means, $\alpha$ and $\beta$ create a cylinder 
with total negative curvature which contradicts the Gauss-Bonnet theorem. (See [12, Lemma 5.6] for a more detailed discussion.)

Since the number of thick components $Q$ is uniformly bounded and $\omega_{T}$ and $\bar{\omega}$ can intersect at most once in each $Q$ we conclude that the total intersection number outside of cylinders $F_{\alpha}$ is uniformly bounded as well. This finishes the proof.

Lemma 3.18 For a quadratic differential $(X, q), \alpha \in \mathcal{S}_{q}^{\leq \tau}$ and $a(q, \tau)$ regular triangulation $T$ we have

$$
\operatorname{twist}_{\alpha}(T, q)=O(1) \text {. }
$$

Proof Let $Q_{1}$ and $Q_{2}$ be the thick subsurfaces of $(X, q)$ glued along the cylinder $F_{\alpha}$ (which by assumption, has a modulus at most $\mathrm{M}_{\tau}$ ), and let $\beta$ be an essential curve in $Q_{1} \cup F_{\alpha} \cup Q_{2}$ that is transverse to $\alpha$ and has the shortest combinatorial $T$-length. A representative for the curve $\beta$ can be constructed using edges of $T$ that intersect either $Q_{1}$ or $Q_{2}$. Consider such a representative traversing the minimum possible number of edges. Let $\gamma$ be a curve transverse to $\alpha$ with the shortest $q$-length. From the definition of relative twisting,

$$
\operatorname{twist}_{\alpha}(T, q) \stackrel{+}{\prec} \mathrm{i}(\beta, \gamma) \text {. }
$$

Hence, it is sufficient to show that $\mathrm{i}(\beta, \gamma)$ is uniformly bounded.

The curve $\gamma$ intersects $\alpha$ once if $Q_{1}=Q_{2}$ and twice otherwise. Its restriction to $Q_{i}$ has a length bounded by $O\left(s_{Q_{i}}\right)$ and its restriction to $F_{\alpha}$ has a length bounded by $\ell_{q}(\alpha)\left(\operatorname{Mod}\left(F_{\alpha}\right)\right.$ is bounded and there is no twisting around $\left.\alpha\right)$ which is less than both $s_{Q_{1}}$ and $s_{Q_{2}}$. An argument similar to that of Lemma 3.17 implies that $\gamma$ intersects any edge of $T$ at most a bounded number of times.

On the other hand, each edge of $T$ appears at most twice along the representative of $\beta$, otherwise a surgery argument would reduce the length of $\beta$. Also, the total number of edges of $T$ is bounded by the topology of $S$. Hence, $\mathrm{i}(\beta, \gamma)$ is uniformly bounded.

\subsection{The number of $(q, \tau)$-regular triangulation}

We now count the number of $(q, \tau)$-regular triangulations near a point in Teichmüller space. We can think of a $(q, \tau)$-regular triangulations on $(X, q)$ as topological objects on $S$, after being pulled back by the marking map $f_{X}: S \rightarrow$ $X$, up to homotopy. That is, we say a $(q, \tau)$-regular triangulation $T$ on $(X, q)$ is equivalent to a $q^{\prime}$-regular triangulation $T^{\prime}$ on $\left(X^{\prime}, q^{\prime}\right)$ if the pre images $f_{X}^{-1}(T)$ and $f_{X^{\prime}}^{-1}\left(T^{\prime}\right)$ are homotopic on $S$. The homotopy does not have to fix the vertices of $T$. For a multi-curve $\mathcal{S}_{0}$, we say $T$ is equivalent to $T^{\prime}$ up 
twisting around $\mathcal{S}_{0}$ if, $T$ is equivalent to $\phi\left(T^{\prime}\right)$ where $\phi$ is a multi-twist with support on curves in $\mathcal{S}_{0}$.

Lemma 3.19 Let $U$ be a ball of radius one in $\mathcal{T}(S)$ centered at $X_{0}$. Then the number of equivalence classes, up to twisting around $\mathcal{S}_{X_{0}}$, of $(q, \tau)$-regular triangulations $T$ on a quadratic differential $(X, q)$ where $X \in U$ is uniformly bounded.

Proof We start with a topological counting statement. Let $\mathcal{S}_{0}=\mathcal{S}_{0}^{c} \cup \mathcal{S}_{0}^{n}$ be a system of curves on $S$. For every subsurface $Q$ in $S \backslash \mathcal{S}_{0}$, let $\mu_{Q}$ be a marking for the subsurface $Q$ in the sense of [33]. That is, $\mu_{Q}$ is a pants decomposition $\left\{\gamma_{1}, \ldots, \gamma_{k}\right\}$ for $Q$ together with a transverse curve $\bar{\gamma}_{i}$ for $0 \leq i \leq k$. Each $\bar{\gamma}_{i}$ is contained in $Q$, intersects $\gamma_{i}$ once or twice and is disjoint from $\gamma_{j}, j \neq i$. Also, for $\alpha \in \mathcal{S}_{0}^{n}$, let $\beta_{\alpha}$ be a curve transverse to $\alpha$ that is disjoint from all other curves in $\mathcal{S}_{0}$ and $\mathrm{i}\left(\beta_{\alpha}, \mu_{Q}\right)=O(1)$. Define

$$
M=\bigcup_{Q} \mu_{Q} \cup \mathcal{S}_{0} \cup\left\{\beta_{\alpha}\right\}_{\alpha \in \mathcal{S}_{0}^{n}} .
$$

Claim Given a set $M$ as above, there is a uniformly bounded number of possibilities for the homotopy class of a triangulation $T$, triangulating $S \backslash \mathcal{S}_{0}^{c}$, where the curves in $M$ and $T$ have representatives with the following properties:

1. curves in $M$ have no self intersections and intersect each other minimally.

2. for any $\alpha \in \mathcal{S}_{0}^{c}, \mathrm{i}(T, \alpha)=0$.

3. for any $\gamma \in \mu_{Q}, \mathrm{i}(T, \gamma)=O(1)$.

4. for $\alpha \in \mathcal{S}_{0}^{n}$, twist $\alpha\left(T, \beta_{\alpha}\right)=O(1)$, and $\mathrm{i}(T, \alpha)=O(1)$.

To see the claim, note that the curves in $M$ divide $S$ into a uniformly bounded number of complementary regions, each one is either a polygon or an annulus parallel to a curve $\alpha \in \mathcal{S}_{0}^{c}$. Choose a representative of the homotopy class of $T$ that intersects curves in $M$ minimally. There are a uniformly bounded number of possibilities for the location of vertices of $T$. Once the vertices of $T$ are fixed, there are a uniformly bounded number of possibilities for any given arc, with end points on these vertices, that can appear as an edge of $T$. This is because there are a uniformly bounded number of possibilities for the intersection pattern of the given arc with the complementary regions. Also, each region is either a polygon where there is a unique arc (up to homotopy) connecting any two edges (or a vertex to an edge) or an annulus neighborhood of a curve $\alpha \in \mathcal{S}_{0}^{c}$ where there are two possibilities (edges of $T$ are simple and disjoint from curves in $\mathcal{S}_{0}^{c}$ ).

It remains to show, that for every $(q, \tau)$-regular triangulation $T_{q}$ on $(X, q)$ where $X \in U$, there is a set of simple closed curves $M_{q}$ so that $T_{q}$ and $M_{q}$ satisfy the above properties and then to bound the number of possibilities for the set $M_{q}$. 
Let $(X, q)$ be a quadratic differential so that $X \in U$. We construct $M_{q}$ as follows: The curves $\mathcal{S}_{q}=\mathcal{S}_{q}^{\geq \tau} \cup \mathcal{S}_{q}^{\leq \tau}$ have a uniformly bounded length in $X_{0}$ hence there are a uniformly bounded number of possibilities for these sets. For each thick subsurface $Q$ of $q$, choose a $q$-short marking $\mu_{Q}$ in $Q$. Curves in $\mu_{Q}$ have a uniformly bounded length on $X$ and hence a uniformly bounded length in $X_{0}$. Hence there are only a uniformly bounded number of choices for these as well. Now for each $\alpha$, let $\beta_{\alpha}^{q}$ be the shortest $q$ transverse curve to $\alpha$. Lemma 3.16 implies that twist $\alpha_{\alpha}\left(X_{0}, \beta_{\alpha}^{q}\right) \stackrel{*}{\prec} \frac{1}{\operatorname{Ext}_{X_{0}}(\alpha)}$. Hence the number of possible choices for $\beta_{\alpha}^{q}$ is of the order of $\frac{1}{\operatorname{Ext}_{x_{0}}(\alpha)}$. Define

$$
M_{q}=\bigcup_{Q} \mu_{Q} \cup \mathcal{S}_{q} \cup\left\{\beta_{\alpha}^{q}\right\}_{\alpha \in \mathcal{S}_{q}^{\leq \tau}}
$$

Bu construction, the total number of possible sets $M_{q}$ chosen as above is of the order of $G\left(X_{0}\right)$. However, up to twisting around $\mathcal{S}_{X_{0}}$ there are only finitely many choices. For a $(q, \tau)$-regular triangulation $T_{q}$ in $(X, q)$, we need to check that the conditions (1)-(4) hold for $T_{q}$ and $M_{q}$. Perturb the $q$-geodesic representative of curves in $M_{q}$ so that they have no self-intersections, intersect each other minimally and the intersection number with $T$ does not increase. Condition (2) follows from the construction of $(q, \tau)$-regular triangulations. Condition (3) follows from Lemma 3.17. The first part of condition (4) is a consequence of Lemma 3.18 and the second part again follows from Lemma 3.17.

\section{Intersection bounds between regular triangulations}

As before, let $\mathcal{Q}(\sigma)$ be the stratum of quadratic differentials of type $\sigma$. In this section, we establish some intersection bounds for $(q, \tau)$-regular triangulations associated to a pair of quadratic differentials that appear at the end points of a geodesic segment in $\mathcal{Q}(\sigma)$.

Recall, from Remark 3.2, that there is an implicit assumption that the constant $\tau$ is large. That is, there is a uniform constant $\tau_{0}$ so that all statements in this section hold as long as $\tau \geq \tau_{0}$. In particular, the implied constant in our estimates do not get worst as $\tau$ gets larger.

\subsection{Notation}

First we need to establish some notations.

1. For a fixed constant $r_{0}$, define $\mathcal{B}(\mathcal{Q}(\sigma), X, \tau)$ to be the set of points $Z \in \mathcal{T}(S)$ so that there is a Teichmüller geodesic 


$$
\mathcal{G}_{Z}:[a, b] \rightarrow \mathcal{Q}^{1} \mathcal{T}(\sigma), \quad \mathcal{G}_{Z}(t)=\left(X_{t}, q_{t}\right),
$$

such that

$$
d_{\mathcal{T}}\left(X_{a}, X\right) \leq r_{0}, \quad d_{\mathcal{T}}\left(X_{b}, Z\right) \leq r_{0}, \quad b-a \leq \tau .
$$

and

$$
\left(X_{t}, q_{t}\right) \in \mathcal{Q}(\sigma)
$$

One could think of $B(\mathcal{Q}(\sigma), X, \tau)$ as a ball of radius $\tau$ centered at $X$, except that one is allowed only to move in the direction of $\mathcal{Q}(\sigma)$. Since $r_{0}$ is fixed, we refer to any constant that depends on $r_{0}$ as a uniform constant. The value of $r_{0}$ will be determined in Sect. 6.2 depending on the choice of the net $\mathcal{N}$.

2. We use the notation of Eq. (5) for $q_{a}$ and denote the flat and expanding annuli associated to a curve $\alpha$ by $E_{\alpha}^{a}, F_{\alpha}^{a}$ and $G_{\alpha}^{a}$ and distances between their boundaries by $e^{a}, f^{a}$ and $g^{a}$. Let $v_{a}$ be an arc of length $f^{a}$ connecting the boundaries of $F_{\alpha}$. Also, let $l^{a}=\ell_{q_{a}}(\alpha)$ and let $d^{a}=\max \left(e^{a}, f^{a}, g^{a}\right)$ be the maximum distance between the boundaries of these annuli. As a consequence of Eqs. (5) and (6) we have

$$
\frac{1}{\operatorname{Ext}_{X}(\alpha)} \stackrel{*}{\prec} \frac{d^{a}}{l^{a}} \quad \text { and } \quad \frac{1}{\operatorname{Ext}_{X}(\alpha)} \stackrel{*}{\succ} \frac{f^{a}}{l^{a}} .
$$

3. Let $T_{a}$ be a $\left(q_{a}, \tau\right)$-regular triangulation and $T_{b}$ be a $\left(q_{b}, \tau\right)$-regular triangulation. The geodesic flow induces a one-to-one correspondence between saddle connections of $q_{a}$ and $q_{b}$. Hence, we can consider $T_{b}$ as a union of saddle connections in $q_{a}$. Then $T_{a}$ and $T_{b}$ have identical vertex sets and their edges are either identical or intersect transversally. The slope of a saddle connection in $q_{a}$ (or in $q_{b}$ ) is a well defined number in the interval $[0, \infty]$.

Definition 4.1 Let $\omega_{a}$ be a saddle connection in $q_{a}$ and let $\omega_{b}$ be a saddle connection in $q_{b}$. We say $\omega_{b}$ intersects $\omega_{a}$ positively, if when considering them both in $q_{a}$ (or $q_{b}$ ), the slope of $\omega_{b}$ is larger than the slope of $\omega_{a}$. We say $\omega_{b}$ intersects $\omega_{a}$ essentially positively if either $\omega_{b}$ intersects $\omega_{a}$ positively or $\mathrm{i}\left(\omega_{a}, \omega_{b}\right)=O(1)$. We use similar terminology for intersection between a saddle connection and a cylinder curve and two cylinder curves.

\subsection{Intersection and twisting bounds between $T_{a}$ and $T_{b}$}

For the rest of this subsection, we assume that $q_{a}$ and $q_{b}, T_{a}$ and $T_{b}$ are as described in the beginning of the section. 
Lemma 4.2 Let $\alpha \in \mathcal{S}_{q_{a}}^{\leq \tau}, \omega_{b} \in T_{b}$ and $\alpha_{b} \in \mathcal{S}_{Z}$, then

$$
\operatorname{twist}_{\alpha}\left(q_{a}, \omega_{b}\right)=O(1) \quad \text { and } \quad \operatorname{twist}_{\alpha}\left(q_{a}, \alpha_{b}\right)=O(1) \text {. }
$$

Similarly, let $\alpha \in \mathcal{S}_{q_{b}}^{\leq \tau}, \omega_{a} \in T_{a}$ and $\alpha_{a} \in \mathcal{S}_{X}$, then

$$
\operatorname{twist}_{\alpha}\left(q_{b}, \omega_{a}\right)=O(1) \quad \text { and } \quad \operatorname{twist}_{\alpha}\left(q_{b}, \alpha_{a}\right)=O(1) .
$$

Proof Let $v_{\alpha}$ be the arc connecting the boundaries of $F_{\alpha}^{a}$ and is perpendicular to them. Then, by definition of $\mathcal{S}_{q}^{\leq \tau}$,

$$
\frac{\ell_{q_{a}}\left(v_{\alpha}\right)}{\ell_{q_{a}}(\alpha)}=\operatorname{Mod}\left(F_{\alpha}^{a}\right) \stackrel{*}{\prec} e^{-2 \tau}
$$

Therefore

$$
\frac{\ell_{q_{b}}\left(v_{\alpha}\right)}{\ell_{q_{b}}(\alpha)} \stackrel{*}{\prec} 1 .
$$

That is, $v_{\alpha}$ twists around $\alpha$ in $q_{b}$ a bounded number of times. But the same is true for $\omega_{b}$. This gives a bound on $\mathrm{i}\left(\omega_{b}, v_{\alpha}\right)$ and thus on twist ${ }_{\alpha}\left(q_{a}, \omega_{b}\right)$. Also, the curve $\alpha_{b}$ is short in $Z$ and hence in $q_{b}$. A short curve can not twist around any other curve. Hence $\mathrm{i}\left(\alpha_{b}, v_{\alpha}\right)$ is uniformly bounded. Which means $\operatorname{twist}_{\alpha}\left(q_{a}, \alpha_{b}\right)$ is uniformly bounded. The proofs of the other two assertions are similar.

Remark 4.3 The main consequence of this lemma is that the twisting condition of Lemma 3.17 is satisfied and can be applied freely.

Lemma 4.4 Let $\omega_{a}$ and $\omega_{b}$ be edges of $T_{a}$ and $T_{b}$ respectively. Then $\omega_{b}$ intersects $\omega_{a}$ essentially positively and

$$
\mathrm{i}\left(\omega_{a}, \omega_{b}\right) \stackrel{*}{\prec} e^{\tau} .
$$

Proof Let $Q_{a}$ be the thick subsurface of $q_{a}$ with the smallest size that intersects $\omega_{a}$ and let $s_{a}$ be the size of the subsurface $Q_{a}$. Recall that, by the definition of a $\left(q_{a}, \tau\right)$-regular triangulation, we have

$$
\ell_{q_{a}}\left(\omega_{a}\right) \stackrel{*}{\prec} s_{a} .
$$

We denote the horizontal and the vertical lengths of $\omega_{a}$ by $x_{a}$ and $y_{a}$. Let $Q_{b}$, $s_{b}, x_{b}$ and $y_{b}$ be similarly defined. The length of $\omega_{a}$ in $Q_{b}$ is

$$
\sqrt{\left(x_{a} e^{\tau}\right)^{2}+\left(y_{a} e^{-\tau}\right)^{2}} \stackrel{*}{`} x_{a} e^{\tau}+y_{a} e^{-\tau} .
$$


If $\mathrm{i}\left(\omega_{a}, \omega_{b}\right)=O(1)$ we are done. Otherwise, Considering $\omega_{a}$ and $\omega_{b}$ in $Q_{b}$, in view of Remark 4.3, Lemma 3.17 implies that

$$
\mathrm{i}\left(\omega_{a}, \omega_{b}\right) \stackrel{*}{\prec} \frac{\ell_{q_{b}}\left(\omega_{a}\right)}{s_{b}}+O(1) .
$$

However, since $\mathrm{i}\left(\omega_{a}, \omega_{b}\right)$ is large, $\frac{\ell_{q_{b}}\left(\omega_{a}\right)}{s_{b}}$ is large and we can incorporate the additive error into the multiplicative error. That is,

$$
\mathrm{i}\left(\omega_{a}, \omega_{b}\right) \stackrel{*}{\prec} \frac{\ell_{q_{b}}\left(\omega_{a}\right)}{s_{b}} \stackrel{*}{*} \frac{x_{a} e^{\tau}+y_{a} e^{-\tau}}{s_{b}} .
$$

Similarly, considering $\omega_{a}$ and $\omega_{b}$ in $Q_{a}$ we get

$$
\mathrm{i}\left(\omega_{a}, \omega_{b}\right) \stackrel{*}{\prec} \frac{\ell_{q_{a}}\left(\omega_{b}\right)}{s_{a}} \stackrel{*}{\longleftarrow} \frac{x_{b} e^{-\tau}+y_{b} e^{\tau}}{s_{a}}
$$

Observing that $x_{a}, y_{a} \stackrel{*}{\prec} s_{a}$ and $x_{b}, y_{b} \stackrel{*}{\prec} s_{b}$, we can multiply the two inequalities and take a square root to get $\mathrm{i}\left(\omega_{a}, \omega_{b}\right) \stackrel{*}{\prec} e^{\tau}$.

Now assume that $\omega_{b}$ does not intersect $\omega_{a}$ positively. This means that the slope of $\omega_{a}$ in $q_{b}$ is larger than the slope of $\omega_{b}$. That is

$$
\frac{y_{a} e^{-\tau}}{x_{a} e^{\tau}} \geq \frac{y_{b}}{x_{b}} \quad \Longrightarrow \quad x_{a} y_{b} e^{2 \tau} \leq x_{b} y_{a}
$$

From the product of inequalities in Eq. (14) and Eq. (15), we have

$$
\begin{aligned}
\mathrm{i}\left(\omega_{a}, \omega_{b}\right)^{2} & \stackrel{*}{\prec} \frac{x_{a} x_{b}+y_{a} y_{b}+x_{a} y_{b} e^{2 \tau}+x_{b} y_{a} e^{-2 \tau}}{s_{a} s_{b}} \\
& \stackrel{*}{\prec} \frac{x_{a} y_{b} e^{2 \tau}}{s_{a} s_{b}} \stackrel{*}{\prec} \frac{x_{b} y_{a}}{s_{a} s_{b}}=O(1) .
\end{aligned}
$$

For a simple closed curve $\alpha$ and a triangulation $T$, we say $T$ intersects $\alpha$ essentially positively if any saddle connection in $T$ intersects any saddle connection in the geodesic representative of $\alpha$ essentially positively.

Lemma 4.5 If $\alpha \in \mathcal{S}_{X}$ and $\alpha \notin \mathcal{S}_{Z}$ then $\alpha$ intersects $T_{b}$ essentially positively and

$$
\operatorname{twist}_{\alpha}(X, Z) \mathrm{i}\left(\alpha, T_{b}\right) \stackrel{*}{\prec} \frac{e^{\tau}}{\sqrt{\operatorname{Ext}_{X}(\alpha)}} .
$$


Similarly, if $\alpha \in \mathcal{S}_{Z}$ and $\alpha \notin \mathcal{S}_{X}$ then $\alpha$ intersects $T_{a}$ essentially positively and

$$
\operatorname{twist}_{\alpha}(X, Z) \mathrm{i}\left(\alpha, T_{a}\right) \stackrel{*}{\prec} \frac{e^{\tau}}{\sqrt{\operatorname{Ext}_{Z}(\alpha)}} .
$$

Proof Let $\alpha$ be a simple closed curve in $\mathcal{S}_{X} \backslash \mathcal{S}_{Z}$. Applying Lemma 3.16 to the pair $X$ and $q_{a}$ and to the pair $Z$ and $q_{b}$, we get

$$
\operatorname{twist}_{\alpha}(X, Z) \stackrel{*}{\prec} \operatorname{twist}_{\alpha}\left(q_{a}, q_{b}\right)+\frac{1}{\operatorname{Ext}_{X}(\alpha)} .
$$

(The term $\frac{1}{\operatorname{Ext}_{Z}(\alpha)}$ is omitted from the right hand side because it is bounded and can be absorbed in the multiplicative error.) Hence, to prove the lemma, it is sufficient to show that the expression $\frac{e^{\tau}}{\sqrt{\operatorname{Ext}_{X}(\alpha)}}$ is an upper-bounds for both

$$
\frac{\mathrm{i}\left(\alpha, T_{b}\right)}{\operatorname{Ext}_{X}(\alpha)} \quad \text { and } \quad \operatorname{twist}_{\alpha}\left(q_{a}, q_{b}\right) \mathrm{i}\left(\alpha, T_{b}\right) .
$$

Let $\omega_{b}$ be an edge of $T_{b}$ and let $Q_{b}$ be the thick subsurface of $q_{b}$ with the smallest size intersecting $\omega_{b}$. Let $s_{b}$ be the size of $Q_{b}$ (thus $\ell_{q_{b}}\left(\omega_{b}\right) \stackrel{*}{\prec} s_{b}$, by the definition of a $\left(q_{b}, \tau\right)$-regular triangulation). Applying Lemma 3.17 to $\omega_{b}$ and $\alpha$ in $q_{b}$, we get

$$
\mathrm{i}\left(\alpha, \omega_{b}\right)-O(1) \stackrel{*}{\prec} \frac{\ell_{q_{b}}(\alpha)}{s_{b}} \leq e^{\tau} \frac{l^{a}}{s_{b}} .
$$

Also, each subsegment of $\omega_{b}$ with end points in $\alpha$ has a length larger than $d^{a}$. Hence,

$$
\mathrm{i}\left(\alpha, \omega_{b}\right)-1 \leq \frac{\ell_{q_{a}}\left(\omega_{b}\right)}{d^{a}} \stackrel{*}{\prec} e^{\tau} \frac{s_{b}}{d^{a}} .
$$

Multiplying these two equations, taking the square root we and summing over all $\operatorname{arcs}$ in $T_{b}$ we get

$$
\mathrm{i}\left(\alpha, T_{b}\right)-O(1) \stackrel{*}{\prec} e^{\tau} \sqrt{\frac{l^{a}}{d^{a}}} \text {. }
$$

In view of Eq. (13), we obtain

$$
\mathrm{i}\left(\alpha, T_{b}\right)-O(1) \stackrel{*}{\prec} e^{\tau} \sqrt{\operatorname{Ext}_{X}(\alpha)} .
$$


Dividing both sides by $\operatorname{Ext}_{X}(\alpha)$ we obtain

$$
\frac{\mathrm{i}\left(\alpha, T_{b}\right)}{\operatorname{Ext}_{X}(\alpha)} \stackrel{*}{\prec} \frac{e^{\tau}+O(1)}{\sqrt{\operatorname{Ext}_{X}(\alpha)}} \stackrel{*}{\prec} \frac{e^{\tau}}{\sqrt{\operatorname{Ext}_{X}(\alpha)}} .
$$

This is the first estimate we required.

We now find an upper-bound for twist ${ }_{\alpha}\left(q_{a}, q_{b}\right) \mathrm{i}\left(\alpha, T_{b}\right)$ by finding separate upper bounds for twist ${ }_{\alpha}\left(q_{a}, q_{b}\right)$ and $\mathrm{i}\left(\alpha, T_{b}\right)$. The argument involved in this new upper bound for $\mathrm{i}\left(\alpha, T_{b}\right)$ is somewhat similar to above, but the two bounds do not imply each other. We need to consider the image of $F_{\alpha}^{a}$ in $q_{b}$ under the Teichmüller geodesic flow. Denote this cylinder by $F_{\alpha}^{b}$, the distance between its boundaries by $f^{b}$ and let $v_{\alpha}^{b}$ be an arc of length $f^{b}$ connecting the boundaries of $F_{\alpha}^{b}$. Let $l^{b}=\ell_{q b}(\alpha)$. Note that the area of $F_{\alpha}^{a}$ and $F_{\alpha}^{b}$ are equal, that is

$$
l^{a} f^{a}=l^{b} f^{b} .
$$

Consider again the arc $\omega_{b}$ in $T_{b}$ of $q_{b}$-length of order $s_{b}$. Then the $q_{b}$-length of every component of $\omega_{b} \cap F_{\alpha}^{b}$ is larger than $f^{b}$. Therefore

$$
\mathrm{i}\left(\alpha, \omega_{b}\right) \stackrel{*}{\prec} \frac{s_{b}}{f_{b}}=\frac{s_{b} l^{b}}{l^{a} f^{a}} .
$$

As before, applying Lemma 3.17 to $\omega_{b}$ and $\alpha$ in $q_{b}$ we have

$$
\mathrm{i}\left(\alpha, \omega_{b}\right) \stackrel{*}{\prec} \frac{l^{b}}{s_{b}}+O(1) \stackrel{*}{\prec} \frac{l^{b}}{s_{b}} .
$$

The reason we can ignore the additive errors here is that since $\alpha$ is not short in $Z$, it has to either be an essential curve in $Q_{b}$ or intersect some boundary curve of $Q_{b}$. In either case, $l^{b} \stackrel{*}{\succ} s_{b}$, in the first case by definition of the size and in the second case by Corollary 3.6. Hence, the additive error can be absorbed into the fraction $\frac{l^{b}}{s_{b}}$. Multiplying the last two inequalities, taking the square root and summing over all arcs in $T_{b}$, we obtain

$$
\mathrm{i}\left(\alpha, T_{b}\right) \stackrel{*}{\prec} \frac{l^{b}}{\sqrt{l^{a} f^{a}}} .
$$

We now argue that a component of $\omega_{b} \cap F_{\alpha}$ can intersect $v_{\alpha}^{b}$ at most a uniformly bounded number of times: since $\alpha$ is not short in $Z, \ell_{q_{b}}(\alpha) \stackrel{*}{\succ} s_{b}$ and $\ell_{q_{b}}\left(\omega_{b}\right) \stackrel{*}{\prec} s_{b}$, which means the intersection number between $\omega_{b}$ and $v_{\alpha}^{b}$ is at most $\frac{\ell_{q_{b}}\left(\omega_{b}\right)}{\ell_{q_{b}}(\alpha)}=O(1)$. Therefore, the relative twisting of $q_{a}$ and $q_{b}$ around $\alpha$ 
is comparable to the intersection number between $v_{\alpha}^{a}$ and $v_{\alpha}^{b}$ which is at most the $q_{a}$-length of $v_{\alpha}^{b}$ divided by the $q_{a}$-length of $\alpha$. That is

$$
\text { twist }_{\alpha}\left(q_{a}, q_{b}\right)-O(1) \stackrel{*}{\asymp} \mathrm{i}\left(v_{\alpha}^{a}, v_{\alpha}^{b}\right) \leq \frac{e^{\tau} f^{b}}{l^{a}} .
$$

Taking a product and using the second part of Eq. (13) we get:

$$
\begin{aligned}
\text { twist }_{\alpha}\left(q_{a}, q_{b}\right) \mathrm{i}\left(\alpha, T_{b}\right)-O\left(\mathrm{i}\left(\alpha, T_{b}\right)\right) & \stackrel{*}{\prec} e^{\tau}\left(\frac{l^{b} f^{b}}{l^{a}}\right) \frac{1}{\sqrt{l^{a} f^{a}}} \\
& \stackrel{*}{\prec} e^{\tau} \sqrt{\frac{f^{a}}{l^{a}}} \stackrel{*}{\prec} \frac{e^{\tau}}{\sqrt{\operatorname{Ext}_{X}(\alpha)}} .
\end{aligned}
$$

By Eq. (18), we have $\mathrm{i}\left(\alpha, T_{b}\right)$ is much smaller than $\frac{e^{\tau}}{\sqrt{\operatorname{Ext}_{X}(\alpha)}}$. Hence,

$$
\operatorname{twist}_{\alpha}\left(q_{a}, q_{b}\right) \mathrm{i}\left(\alpha, T_{b}\right) \stackrel{*}{\prec} \frac{e^{\tau}}{\sqrt{\operatorname{Ext}_{X}(\alpha)}} \text {. }
$$

The estimate in the Lemma follows from Eqs. (16), (18) and (21).

It remains to show that $\omega_{b}$ and $\alpha$ intersect essentially positively. Let $\omega_{a}$ be a saddle connection of $\alpha$ that intersects $\omega_{b}$ many times. Then, by Lemma 3.17, $\ell_{q_{b}}\left(\omega_{a}\right) \geq s_{b}$. However, $\ell_{q_{b}}\left(\omega_{b}\right) \stackrel{*}{\prec} s_{b}$ and hence $\ell_{q_{b}}\left(\omega_{a}\right) \stackrel{*}{\succ} l_{q_{b}}\left(\omega_{b}\right)$. If the slope of $\omega_{\alpha}$ was smaller than $\omega_{b}$ (say in $q_{b}$ ) then we would also have $\ell_{q_{a}}\left(\omega_{a}\right) \stackrel{*}{\succ}$ $\ell_{q_{a}}\left(\omega_{b}\right)$. Hence, $\omega_{a}$ intersects $\omega_{b}$ at most twice (its length is less than $d^{a}$ ). This proves that $\omega_{b}$ intersects $\omega_{\alpha}$ essentially positively. But this is true for every saddle connection of $\alpha$. Thus $\omega_{b}$ intersects $\alpha$ essentially positively. The case when $\alpha \in \mathcal{S}_{Z}$ can be treated similarly.

Lemma 4.6 Let $\alpha \in \mathcal{S}_{X}$ and $\alpha_{0} \in \mathcal{S}_{Z}$. Then

1. If $\alpha_{0} \neq \alpha$, then

$$
\mathrm{i}\left(\alpha, \alpha_{0}\right) \text { twist }_{\alpha_{0}}(X, Z) \text { twist }_{\alpha}(X, Z) \stackrel{*}{\prec} \frac{e^{\tau}}{\sqrt{\operatorname{Ext}_{X}(\alpha) \operatorname{Ext}_{Z}\left(\alpha_{0}\right)}},
$$

2. If $\alpha=\alpha_{0}$, then

$$
\operatorname{twist}_{\alpha}(X, Z) \stackrel{*}{\prec} \frac{e^{\tau}}{\sqrt{\operatorname{Ext}_{X}(\alpha) \operatorname{Ext}_{Z}\left(\alpha_{0}\right)}} .
$$

Proof It is enough to prove that

$$
\operatorname{twist}_{\alpha}(X, Z) \sqrt{\operatorname{Ext}_{X}(\alpha) \operatorname{Ext}_{Z}(\alpha)} \stackrel{*}{\prec} e^{\tau} \text {. }
$$


If $\alpha=\alpha_{0}$, this is equivalent to Eq. (22). Also, if $\alpha \neq \alpha_{0}$ and $\alpha \in S_{Z}$, then the inequality (1) trivially holds (the left hand side is 0 ). Otherwise, from Theorem 3.14 (estimating $\operatorname{Ext}_{Z}(\alpha)$ ) we have

$$
\mathrm{i}\left(\alpha, \alpha_{0}\right) \sqrt{\operatorname{Ext}_{Z}\left(\alpha_{0}\right)} \text { twist }_{\alpha_{0}}(X, Z) \stackrel{*}{\prec} \sqrt{\operatorname{Ext}_{Z}(\alpha)} .
$$

Multiplying the above equation to Eq. (22) we obtain part (1) of the lemma.

By Corollary 3.15, (replace $X$ with $Z, \gamma$ with $\alpha$ and $\beta$ with $\beta_{0}$ ) there always exits a simple closed curve $\beta_{0}$ so that twist ${ }_{\alpha}\left(\beta_{0}, Z\right)=O(1)$ and

$$
\sqrt{\operatorname{Ext}_{Z}\left(\beta_{0}\right) \operatorname{Ext}_{Z}(\alpha)} \stackrel{*}{\prec} \mathrm{i}\left(\alpha, \beta_{0}\right) .
$$

On the other hand, from Theorem 2.1 we have

$$
\sqrt{\operatorname{Ext}_{X}\left(\beta_{0}\right)} \stackrel{*}{\prec} e^{\tau} \sqrt{\operatorname{Ext}_{Z}\left(\beta_{0}\right)},
$$

and from Theorem 3.14 (this time estimating the length of $\beta_{0}$ is $X$ ) we have

$$
\mathrm{i}\left(\alpha, \beta_{0}\right) \text { twist }_{\alpha}\left(X, \beta_{0}\right) \sqrt{\operatorname{Ext}_{X}(\alpha)} \stackrel{*}{\prec} \sqrt{\operatorname{Ext}_{X}\left(\beta_{0}\right)} .
$$

Since twist $\alpha_{\alpha}\left(\beta_{0}, Z\right)=O(1)$, we can replace twist ${ }_{\alpha}\left(X, \beta_{0}\right)$ with twist ${ }_{\alpha}(X, Z)$ in the above inequality. Now, Eq. (22) is obtained by successive substitution using Eqs. (23), (24), (25) and (26).

\subsection{Relations between intersections numbers}

So far, we have provided upper-bounds for the intersection numbers between the edges of $T_{a}$ and the edges of $T_{b}$. But these intersection numbers are not independent. The fact that the edges in $T_{a}$ intersect edges in $T_{b}$ essentially positively allows us to find relations between these intersection numbers. In this section we will describe these relations. There are two kinds of relations.

Lemma 4.7 For every triangle in $T_{a}$ with edges $\omega_{1}, \omega_{2}$ and $\omega_{3}$, there are sings $\varsigma_{1}, \varsigma_{2}, \varsigma_{3} \in\{-1,+1\}$ so that, for every edge $\omega_{b}$ in $T_{b}$ (respectively, for any $\alpha_{b} \in \mathcal{S}_{q_{b}}^{\geq \tau}$ ), we have the relation:

$$
\sum_{i=1,2,3} \varsigma_{i} \mathrm{i}\left(\omega_{i}, \omega_{b}\right)=O(1) \quad\left(\text { respectively, } \sum_{i=1,2,3} \varsigma_{i} \mathrm{i}\left(\omega_{i}, \alpha_{b}\right)=O(1)\right) .
$$

The additive error depends on the constant involved in the definition of essential positively. 
Proof There is a leaf of the vertical foliation that passes through a vertex of the given triangle before entering it. Assume this leaf intersects the interior of $\omega_{3}$ and makes an acute angle with $\omega_{1}$ inside of the triangle. We claim that, since $\omega_{b}$ intersects $\omega_{1}$ essentially positively, the number of sub-arcs of $\omega_{b}$ going from $\omega_{1}$ to $\omega_{2}$ is uniformly bounded. This is because either the slope of $\omega_{b}$ is larger than the slope of $\omega_{1}$ and every time $\omega_{b}$ intersects $\omega_{1}$ it has to intersect $\omega_{3}$ next, or it intersect $\omega_{1}$ a bounded number of times. Hence, we have

$$
\mathrm{i}\left(\omega_{1}, \omega_{b}\right)+\mathrm{i}\left(\omega_{2}, \omega_{b}\right)=\mathrm{i}\left(\omega_{3}, \omega_{b}\right)+O(1) .
$$

Note that the signs $\varsigma_{1}=1, \varsigma_{2}=1$ and $\varsigma_{3}=-1$ depend only on the triangle and are independent of $\omega_{b}$. The proof for $\alpha_{b}$ is similar.

For each $\alpha \in \mathcal{S}_{q_{a}}^{\geq \tau}$, consider a saddle connection $\beta_{\alpha}$ connecting the boundaries of $F_{\alpha}$. Let

$$
U_{a}=T_{a} \cup \bigcup_{\alpha \in \mathcal{S}_{q a}^{\geq \tau}} \beta_{\alpha}
$$

We can choose the $\operatorname{arcs} \beta_{\alpha}$ so that twist ${ }_{\alpha}\left(q_{a}, U_{a}\right)=O(1)$. After orienting the arcs in $U_{a}$, we can think of them as elements of $H_{1}(S, \Sigma)$ where $\Sigma$ is the set critical points of $q_{a}$. In fact, arcs in $U_{a}$ generate $H_{1}(S, \Sigma)$.

Lemma 4.8 Assume that the vertical foliation of $q_{a}$ is not orientable. Then, there is a set $\mathcal{B}$ of edges of $U_{a}$ and for $\omega \in \mathcal{B}$ there is a sign $\varsigma_{\omega} \in\{-1,+1\}$ so that, for every $\omega_{b}$ in $T_{b}$ (respectively, for any $\alpha_{b} \in \mathcal{S}_{q_{b}}^{\geq \tau}$ ), we have the relation:

$$
\sum_{\omega \in \mathcal{B}} \varsigma_{\omega} \mathrm{i}\left(\omega, \omega_{b}\right)=O(1) \quad\left(\text { respectively, } \quad \sum_{\omega \in \mathcal{B}} \varsigma_{\omega} \mathrm{i}\left(\omega, \alpha_{b}\right)=O(1)\right) .
$$

Furthermore, this relation is independent of all the relations in Lemma 4.7.

Proof Choose a minimum number of edges of $U_{a}$ so that the complement is simply connected. Denote the set of all these edges by $\overline{\mathcal{B}}$ and orient them in some arbitrary way. Minimality implies that the compliment $P$ is connected. We can visualize $P$ as a polygon in $\mathbb{C}$ with the vertical foliation parallel to the imaginary axis. Each edge of $\overline{\mathcal{B}}$ has two representatives in the boundary of $P$. The two vectors are equal up to a multiplication by \pm 1 . Let $\mathcal{B}$ be the subset of $\overline{\mathcal{B}}$ where the two representatives are negatives of each other (Fig. 5). Note that $\mathcal{B}$ is non-empty since the vertical foliation in $q_{a}$ is not orientable.

Now consider a double cover of $q_{a}$ constructed as follows. Take a second copy $P^{\prime}$ of $P$. Glue the edges that were not in $\mathcal{B}$ as before and glue the edges 
Fig. 5 Polynomial $P$. The set $\mathcal{B}=\{a, b, c\}$
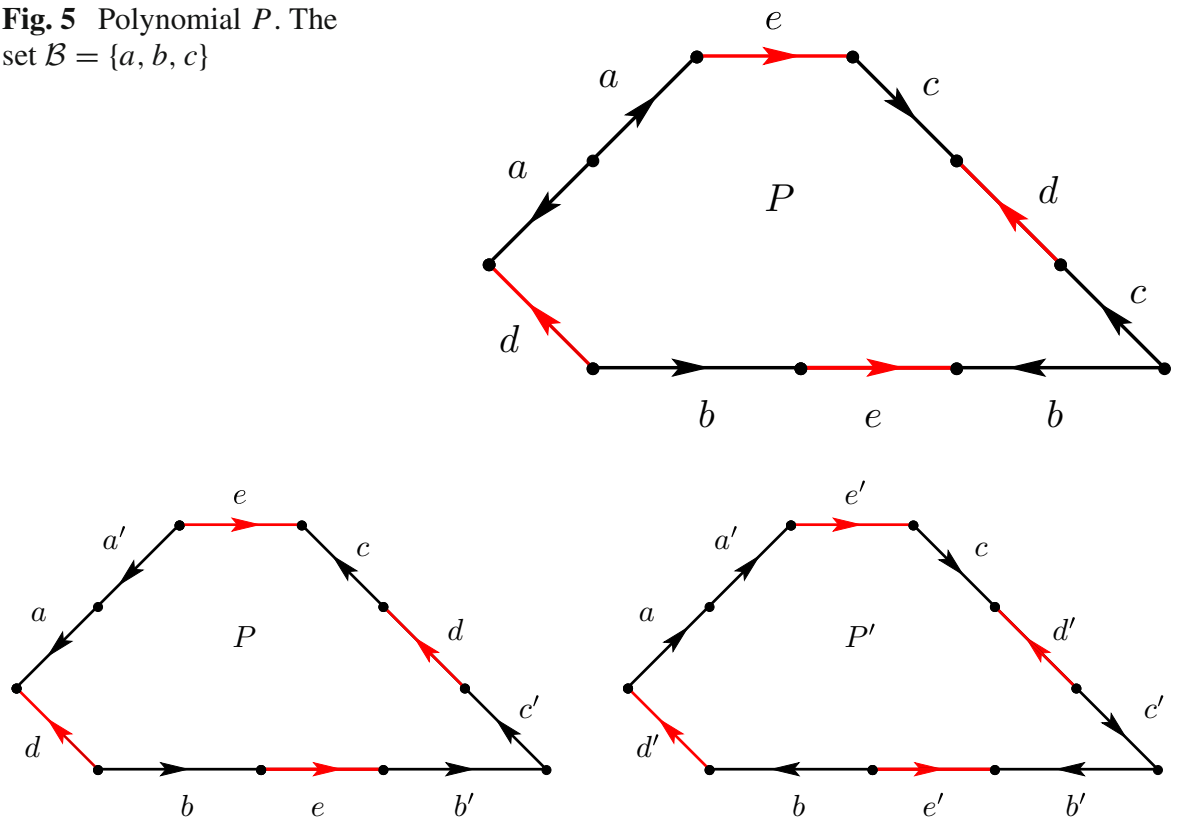

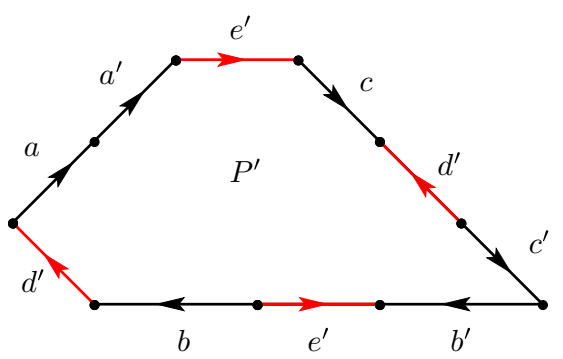

Fig. 6 Polynomials $P$ and $P^{\prime}$. The set $\widetilde{\mathcal{B}}=\left\{a, a^{\prime}, b, b^{\prime}, c, c^{\prime}\right\}$

in $\mathcal{B}$ to the corresponding edge in $P^{\prime}$. Let $\widetilde{\mathcal{B}}$ be the set of lifts of edges in $\mathcal{B}$ to this cover. We now orient edges in $\widetilde{\mathcal{B}}$ so that, for every $\tilde{\omega} \in \widetilde{\mathcal{B}}, P$ is in the same side of $\tilde{\omega}$ (say, the left side). Denote this double cover by $\tilde{q}_{a}=P \cup P^{\prime}$.

Let $\widetilde{S}$ be the underlying surface for $\tilde{q}_{a}$ and $\widetilde{\Sigma}$ be the pre-image of $\Sigma$. Considering oriented saddle connections as elements of $H_{1}(\widetilde{S}, \widetilde{\Sigma})$ we let $\hat{\mathrm{i}}(\cdot, \cdot)$ denote the algebraic intersection number. Note that $\tilde{q}_{a}$ is the unique double cover of $q_{a}$ where $\tilde{q}_{a}$ is a square of an abelian differential. Hence, for every two oriented saddle connections $\tilde{\omega}$ and $\tilde{\omega}^{\prime}$ in $\tilde{q}_{a}$, all the intersection points have the same signature. That is,

$$
\mathrm{i}\left(\tilde{\omega}, \tilde{\omega}^{\prime}\right)=\left|\hat{\mathrm{i}}\left(\tilde{\omega}, \tilde{\omega}^{\prime}\right)\right| .
$$

Consider $\omega \in \mathcal{B}$ and its lift $\tilde{\omega}$. Note that $\tilde{\omega}$ has an orientation and hence is identified with vector in $\mathbb{C}$. We define $\varsigma_{\omega}$ to be +1 if $\tilde{\omega}$ has a positive $x$ coordinate and -1 otherwise. Let $\omega_{b} \in T_{b}$ and let $\tilde{\omega}_{b}$ be a lift of $\omega_{b}$. We choose an orientation for $\tilde{\omega}_{b}$ so it has a positive $y$-coordinate. We will show that

$$
\sum_{\omega \in \mathcal{B}} \varsigma_{\omega} \mathrm{i}\left(\omega, \omega_{b}\right)=O(1)
$$

Consider an intersection point of $\tilde{\omega}_{b}$ and $\tilde{\omega}$ where $\varsigma_{\omega}=1$. If the absolute value of the slope of $\tilde{\omega}_{b}$ is larger than that of $\tilde{\omega}$ then $\tilde{\omega}_{b}$ is to the left of $\tilde{\omega}$ and 
hence $\tilde{\omega}$ intersects $\tilde{\omega}_{b}$ with a positive signature. Otherwise, $\tilde{\omega}$ and $\tilde{\omega}_{b}$ intersect a uniformly bounded number of times ( $\tilde{\omega}_{b}$ and $\tilde{\omega}$ intersect essentially positively) (Fig. 6). The opposite of this is true if $\varsigma_{\omega}=-1$; either $\tilde{\omega}$ intersects $\tilde{\omega}_{b}$ with a negative signature or a uniformly bounded number of times. If $\tilde{\omega}, \tilde{\omega}^{\prime} \in \widetilde{\mathcal{B}}$ are lifts of the same arc $\omega \in \mathcal{B}$ then, choosing orientations for $\tilde{\omega}$ and $\tilde{\omega}^{\prime}$ as above, we have

$$
\mathrm{i}\left(\omega, \omega_{b}\right)=\mathrm{i}\left(\tilde{\omega}, \tilde{\omega}_{b}\right)+\mathrm{i}\left(\tilde{\omega}^{\prime}, \tilde{\omega}_{b}\right) \succsim \varsigma_{\omega}\left(\hat{\mathrm{i}}\left(\tilde{\omega}, \tilde{\omega}_{b}\right)+\hat{\mathrm{i}}\left(\tilde{\omega}^{\prime}, \tilde{\omega}_{b}\right)\right) .
$$

To reiterate, this is because the number of intersection points that do not have the same sign as $\varsigma_{\omega}$ is uniformly bounded.

But arcs in $\widetilde{\mathcal{B}}$ separate $\tilde{q}_{a}$. Thus,

$$
\sum_{\tilde{\omega} \in \widetilde{\mathcal{B}}} \hat{\mathrm{i}}\left(\tilde{\omega}, \tilde{\omega}_{b}\right) \leq 1
$$

This is because every time $\tilde{\omega}_{b}$ exits $P$ it intersects the boundary with the opposite signature than when it enters it. The sum is not necessarily zero because $\tilde{\omega}_{b}$ may start inside $P$ and end in $P^{\prime}$. Therefore, summing Eq. (29) over $\omega \in \mathcal{B}$, we get

$$
\sum_{\omega \in \mathcal{B}} \varsigma_{\omega} \hat{\mathrm{i}}\left(\omega, \omega_{b}\right)=O(1)
$$

The proof for the case of a simple closed curve $\alpha_{b} \in \mathcal{S}_{q_{b}}^{\geq \tau}$ is similar.

Finally, we note that the relations of the type (27) are also relations in the relative homology with $\mathbb{Z}_{2}$-coefficients. But the edges in $\mathcal{B}$ are independent in $\mathbb{Z}_{2}$-relative homology. Hence, this new relation is independent from the previous ones.

\section{Main counting statement}

This section contains the main combinatorial counting arguments with the goal of proving Theorem 5.1. Recall the definition of $B(\mathcal{Q}(\sigma), X, \tau)$ from Sect. 4.1. Define

$$
B_{j}(\mathcal{Q}(\sigma), X, \tau) \subset B(\mathcal{Q}(\sigma), X, \tau)
$$

to be the set of points $Z \in \mathcal{T}(S)$ so that, for the associated quadratic differentials $q_{a}$ and $q_{b}$, there is a $\left(q_{a}, \tau\right)$-regular triangulation $T_{a}$ and a $\left(q_{b}, \tau\right)$-regular 
triangulation $T_{b}$ that have $j$ common homologically independent saddle connections. Now let,

$$
B(\mathcal{Q}(\sigma), X, Y, \tau)=B(\mathcal{Q}(\sigma), X, \tau) \cap(\Gamma(S) \cdot Y),
$$

and

$$
B_{j}(\mathcal{Q}(\sigma), X, Y, \tau)=B_{j}(\mathcal{Q}(\sigma), X, \tau) \cap(\Gamma(S) \cdot Y)
$$

That is, $B_{j}(\mathcal{Q}(\sigma), X, Y, \tau)$ is the intersection of the orbit of $Y$ with $B_{j}(\mathcal{Q}(\sigma), X, \tau)$. Also, recall from Sect.2.6 that (when $\mathcal{S}_{X}$ is empty, $G(X)=$ 2):

$$
G(X)=1+\prod_{\alpha \in \mathcal{S}_{X}} \frac{1}{\sqrt{\operatorname{Ext}_{X}(\alpha)}} \stackrel{*}{\longleftarrow} \prod_{\alpha \in \mathcal{S}_{X}} \frac{1}{\sqrt{\operatorname{Ext}_{X}(\alpha)}} .
$$

Notice that if $\mathfrak{g} \cdot Y \in B_{j}(\mathcal{Q}(\sigma), X, Y, \tau)$ then $\mathfrak{g}^{-1} \cdot X \in B_{j}(\mathcal{Q}(\sigma), Y, X, \tau)$. Thus, the number of points in $B_{j}(\mathcal{Q}(\sigma), X, Y, \tau)$ is the same as the number of points in $B_{j}(\mathcal{Q}(\sigma), Y, X, \tau)$. We prove the following upper-bound for the size of $B_{j}(\mathcal{Q}(\sigma), Y, X, \tau)$ :

Theorem 5.1 Consider the stratum $\mathcal{Q}(\sigma)$. Given $X, Y \in \mathcal{T}(S)$

$$
\left|B_{j}(\mathcal{Q}(\sigma), X, Y, \tau)\right| \stackrel{*}{\prec} \tau^{\left|\mathcal{S}_{X}\right|+\left|\mathcal{S}_{Y}\right|} e^{(h-j) \tau} G(X) G(Y),
$$

where $h=\frac{\operatorname{dim} \mathcal{Q}(\sigma)}{2}$.

Remark 5.2 First we make a few remarks

1. If, in the definition of $B_{j}(\mathcal{Q}(\sigma), X, Y, \tau)$, we replace the assumption on the number of common homologically independent saddle connections with an assumption on the number of common homologically independent simple closed curves, the same statement would still holds. However, the theorem is strictly stronger. For example, assume $\mathcal{S}_{X} \cap \mathcal{S}_{Y}$ contains only one homologically trivial simple closed curves $\alpha$. We can still conclude that $j \geq 1$ because the geodesic representative of $\alpha$ in any quadratic differential $q$ contains a (homologically) non-trivial arc. That is, the number points $Y$, where the geodesic connecting $X$ to $Y$ follows $\mathcal{Q}(\sigma)$ and contains a short curve throughout, is smaller than expected even when $\alpha$ is a homologically trivial curve.

2. The statement appears to be correct even without the term $\tau^{\left|\mathcal{S}_{X}\right|+\left|\mathcal{S}_{Y}\right|}$. However, the proof would become significantly more complicated. 


\subsection{Sketch of the proof Theorem 5.1}

Here is a an outline of our strategy:

1. We define a notion of a marking for the surface $S$ and what it means for a marking to have a bounded length in a Riemann surface $X$. A marking contains a partial triangulation of $S$, a set of short simple closed curves with their lengths and some twisting information. Fixing a Riemann surface $X$, every quadratic differential $q$ where the underlying conformal structure is near $X$ defines a marking that has a bounded length in $X$. A marking takes the lengths of the short simple closed curves and the twisting information around short cylinder curves from $X$ and the triangulation and twisting around the non-cylinder short simple closed curves from $q$. Up to some twisting information, there are a uniformly bounded number of markings that have bounded length in a given Riemann surface $X$.

2. Fixing a marking $\Delta_{0}$, a relation is a formal linear combination of edges of $\Delta_{0}$ with integer coefficients. Given $\Delta_{0}$ and $\Delta_{1}$ and a set of relations $\mathcal{R}$ we will define a set $M_{\mathcal{R}}\left(\Delta_{0}, \Delta_{1}, \tau\right)$ consisting of all markings $\Delta$ such that $\Delta$ is a homeomorphic image of $\Delta_{1}$, its weighted intersection number with $\Delta_{0}$ is less than $e^{\tau}$ and so that the intersection patterns between $\Delta$ and $\Delta_{0}$ satisfy the relations in $\mathcal{R}$. The weights depend on the length and the twisting information of each short simple closed curve. This is similar to assuming that there is a geodesic segment in a the stratum $\mathcal{Q}(\sigma)$ starting near $X$ and ending near $Y$. Lemma 5.8 provides and upper-bound for the number of elements in $M_{\mathcal{R}}\left(\Delta_{0}, \Delta_{1}, \tau\right)$.

3. We then let $\mathcal{R}$ be the set of relation of the type described in Lemma 4.7 and Lemma 4.8. Each $Z \in B_{j}(\mathcal{Q}(\sigma), X, Y, \tau)$ can then be mapped to a marking in $\Delta \in M_{\mathcal{R}}\left(\Delta_{0}, \Delta_{1}, \tau\right)$ for some marking $\Delta_{1}$ that has bounded length in $Y$ and some marking $\Delta_{0}$ that has both a bounded length and a bounded twisting in $X$. This map is finite-to-one except for some twisting information. An estimate for the number of possible markings $\Delta_{0}$ and $\Delta_{1}$ provides the desired upper-bound for the size of $B_{j}(\mathcal{Q}(\sigma), X, Y, R)$.

As is apparent from the outline, the main complication is to keep careful track of all the different twisting informations. Otherwise, the argument is relatively elementary.

\subsection{Markings on $S$}

Fix a set of points $\Sigma$ on $S$. A partial triangulation $T$ of $S$ with the vertex set $\Sigma$ is an embedding of a graph to $S$ where vertices are mapped onto $\Sigma$ and the complementary components are either triangles or annuli. Even though the vertex set is fixed, we think of $T$ as representing a free homotopy class of 
triangulations. We say a curve $\gamma$ is carried by $T$ if the free homotopy class of $\gamma$ can be represented by tracing the edges of $T$. We define a combinatorial length of a simple closed curve $\gamma$ in $S$ to be the minimum number of arcs of $T$ that can appear in a representative of $\gamma$ and we denote it by $\ell_{T}(\gamma)$.

Recall that a set of curves fill a subsurface $Q$ os $S$ if every essential curve in $Q$ intersects one of these curves. We say a partial triangulation $T$ fills a subsurface $Q$ of $S$ if, again, every essential curve in $Q$ intersects $T$ (their free homotopy classes do not have disjoint representatives). The two notions are related:

Lemma 5.3 There is a constant B such that, if $T$ fills a subsurface $Q$ of $S$, then the set of simple closed curves $\gamma$ carried by $T$ with $\ell_{T}(\alpha) \leq \mathrm{B}$ also fill the subsurface $Q$.

Definition 5.4 A marking $\Delta=\Delta(\mathcal{S},\{E(\alpha)\}, T)$ for $S$ is:

- a free homotopy class of oriented curve system $\mathcal{S}$ (pairwise disjoint curves) together with a notion of zero twisting for each curve $\alpha \in \mathcal{S}$, (that is, the expression twist ${ }_{\alpha}(\Delta, \cdot)$ makes sense),

- a length $E(\alpha)$ associated to each simple closed curve $\alpha \in \mathcal{S}$, and

- a homotopy class of a partial triangulation $T$ with the vertex set $\Sigma$ such that the core curve of any annulus in the complement of $T$ is in $\mathcal{S}$.

- for each $\alpha \in \mathcal{S}$ intersecting $T$, twist $\alpha(\Delta, T)=O(1)$.

We denote the set of simple closed curves that are disjoint from $T$ by $\mathcal{S}^{c}$ and the remaining curves in $\mathcal{S}$ by $\mathcal{S}^{n}$ (the set $\mathcal{S}^{c}$ is a place holder for large cylinder curves and the set $\mathcal{S}^{n}$ is a place holder for non-cylinder curves or small cylinder curves).

We say a marking $\Delta=(\mathcal{S},\{E(\alpha)\}, T)$ has a bounded length in $X$ if:

1. $\mathcal{S}=\mathcal{S}_{X}$.

2. For $\alpha \in \mathcal{S}, E(\alpha)=\operatorname{Ext}_{X}(\alpha)$.

3. For $\alpha \in \mathcal{S}^{c}$, $\operatorname{twist}_{\alpha}(\Delta, X)=O(1)$.

4. For each simple closed curve $\gamma \notin \mathcal{S}_{X}$ that is disjoint from $\mathcal{S}_{X}, \ell_{X}(\gamma) \stackrel{*}{ }$ $\ell_{T}(\gamma)$.

We say $\Delta$ has bounded length in $X$ with $\tau$-bounded twist if we further have

5. For $\alpha \in \mathcal{S}^{n}$, twist $_{\alpha}(\Delta, X)=O(\tau)$.

Example 5.5 We continue Example 3.4 of a surface $(X, q)$ described by a gluing of a polygon in $\mathbb{R}^{2}$. As it was discussed, there are two thick subsurfaces in the complement of curves $\alpha$ and $\beta$ (Fig. 2). A (q, $\tau)$-regular triangulation of $(X, q)$ is depicted in Fig. 7. Here $\mathcal{S}_{q}^{\leq \tau}=\{\alpha\}$ and $\mathcal{S}_{q}^{\geq \tau}=\{\beta\}$.

Here a marking $\Delta$ that has bounded length in $X$ can be obtained as follows: The set $\mathcal{S}$ is the set $\{\alpha, \beta\}$ of short curves in $X$ (depicted as blue curves in 


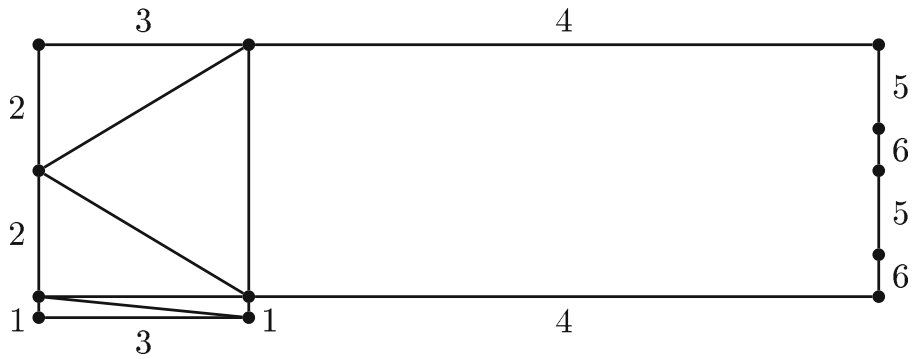

Fig. 7 A $(q, \tau)$-regular triangulation
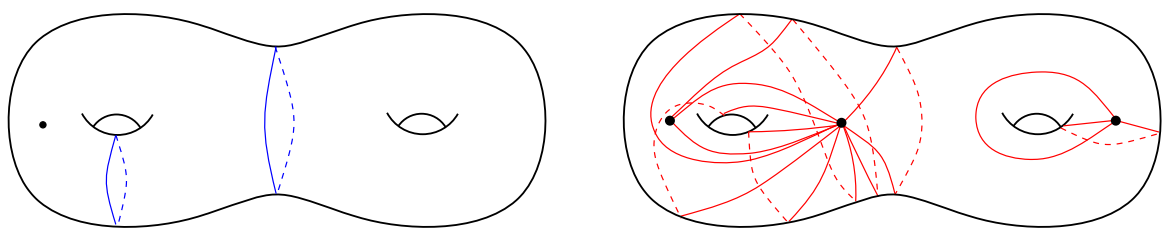

Fig. 8 The curves and the triangulation in the marking $\Delta$

Fig. 8), the triangulation $T$ is the $(q, \tau)$-regular triangulation (depicted as the red triangulation) and $E(\alpha)$ and $E(\beta)$ are the extremal length of $\alpha$ and $\beta$ in $X$ respectively. The condition (4) for $\Delta$ to have a bounded length in $X$ is a consequence of $T$ being a $(q, \tau)$-regular triangulation.

Lemma 5.6 Let $M(X, \tau)$ be the set of markings $\Delta$ that have a bounded length in $X$ with $\tau$-bounded twist. Then

$$
|M(X, \tau)| \stackrel{*}{\prec} \tau^{\left|\mathcal{S}_{X}\right|} .
$$

Proof The set $\mathcal{S}$ and the lengths $\{E(\alpha)\}$ and the twisting around curves in $\mathcal{S}^{c}$ are determined by definition. By Lemma 3.19, there is a uniformly bounded number of possibilities for $T$ up to twisting around curves in $\mathcal{S}^{n}$. But each of these twisting parameters is bounded by multiple of $\tau$ (condition (5) in the definition Definition 5.4). This finishes the proof.

Definition 5.7 Consider the markings

$$
\Delta=\{\mathcal{S},\{E(\alpha)\}, T\} \text { and } \Delta_{0}=\left\{\mathcal{S}_{0},\left\{E_{0}\left(\alpha_{0}\right)\right\}, T_{0}\right\} .
$$

Recall that $T$ and $T_{0}$ have the same vertex set $\Sigma$. For every $\alpha \in \mathcal{S}^{c}$, let $\beta_{\alpha}$ be an arc with end point in $\Sigma$ and disjoint from $T$ that crosses $\alpha$ so that $T \cup \beta_{\alpha}$ has bounded twisting around $\alpha$. Denote

$$
U=T \cup \bigcup_{\alpha \in \mathcal{S}^{c}} \beta_{\alpha} .
$$


Let $\mathbb{R}[U]$ be the vector space of formal sums with real coefficient of edges in $U$. Let $\mathcal{R}$ be a finite subset of $\mathbb{R}[U]$ with integer coefficients. We define the set

$$
M_{\mathcal{R}}\left(\Delta, \Delta_{0}, \tau\right)
$$

to be the set of markings $\bar{\Delta}=\{\overline{\mathcal{S}},\{\bar{E}(\bar{\alpha})\}, \bar{T}\}$ such that:

(I) $\bar{\Delta}$ is a homeomorphic image of $\Delta_{0}$, and for every $\bar{\alpha} \in \overline{\mathcal{S}}^{c}$ that is the image of $\alpha_{0} \in \mathcal{S}_{0}^{c}$, we have $E_{0}\left(\alpha_{0}\right)=\bar{E}(\bar{\alpha})$.

(II) For every element $\sum a_{\omega} \omega \in \mathcal{R}$ and every $\operatorname{arc} \bar{\omega} \in \bar{T}$ (respectively, $\bar{\alpha} \in \overline{\mathcal{S}}^{c}$ ), we have

$$
\sum_{\omega \in U} a_{\omega} \mathrm{i}(\omega, \bar{\omega})=O(1), \quad\left(\text { respectively, } \quad \sum_{\omega} a_{\omega} \mathrm{i}(\omega, \bar{\alpha})=O(1)\right) .
$$

(III) Given $\alpha \in \mathcal{S}^{c}, \bar{\alpha} \in \overline{\mathcal{S}}^{c}, \omega \in T$ and $\bar{\omega} \in \bar{T}$, we have the following bounds on the intersection numbers:

$$
\begin{aligned}
\mathrm{i}(\omega, \bar{\omega}) \stackrel{*}{\prec} e^{\tau} \\
\operatorname{twist}_{\bar{\alpha}}(\Delta, \bar{\Delta}) \sqrt{E(\bar{\alpha})} \mathrm{i}(\bar{\alpha}, T) \stackrel{*}{\prec} e^{\tau} \\
\operatorname{twist}_{\alpha}(\Delta, \bar{\Delta}) \sqrt{E(\alpha)} \mathrm{i}(\alpha, \bar{T}) \stackrel{*}{\prec} e^{\tau} \\
\mathrm{i}(\alpha, \bar{\alpha}) \operatorname{twist}_{\alpha}(\Delta, \bar{\Delta}) \operatorname{twist}_{\bar{\alpha}}(\Delta, \bar{\Delta}) \sqrt{\bar{E}(\bar{\alpha}) E(\alpha)} \stackrel{*}{\prec} e^{\tau}
\end{aligned}
$$

and finally if $\alpha=\bar{\alpha} \in \mathcal{S}^{c} \cap \overline{\mathcal{S}}^{c}$ we have:

$$
\operatorname{twist}_{\alpha}(\Delta, \bar{\Delta}) \sqrt{E(\alpha) \bar{E}(\bar{\alpha})} \stackrel{*}{\prec} e^{\tau} .
$$

Note that the partial triangulations in $\Delta$ and $\bar{\Delta}$ are defined up to homotopy. By above intersection bounds we mean that the homotopy class of two partial triangulations have representations with vertex set $\Sigma$ so that the above bounds hold simultaneously.

Let $\langle\mathcal{R}\rangle$ be the subspace of $\mathbb{R}[U]$ generated by elements in $\mathcal{R}$. We give the following upper bound for such markings:

Lemma 5.8 Let $h_{\mathcal{R}}=\operatorname{dim}(\mathbb{R}[U] /\langle\mathcal{R}\rangle)$. Then

$$
\left|M_{\mathcal{R}}\left(\Delta, \Delta_{0}, \tau\right)\right| \stackrel{*}{\prec} e^{h_{\mathcal{R}} \tau} \prod_{\alpha \in \mathcal{S}^{c}} \frac{1}{\sqrt{E(\alpha)}} \prod_{\alpha_{0} \in \mathcal{S}_{0}^{c}} \frac{1}{\sqrt{E_{0}\left(\alpha_{0}\right)}} .
$$


Proof For $\bar{\Delta} \in M_{\mathcal{R}}\left(\Delta, \Delta_{0}, \tau\right)$ consider the weighted graphs

$$
\bar{W}=\bar{T}+\sum_{\bar{\alpha} \in \overline{\mathcal{S}}^{c}} m(\bar{\alpha}, \bar{\Delta}) \bar{\alpha},
$$

where the weights on the edges of $\bar{T}$ are 1 and the weight $m(\bar{\alpha}, \bar{\Delta}) \in \mathbb{N}$ are defined to be

$$
m(\bar{\alpha}, \bar{\Delta})=\left\lfloor\operatorname{twist}_{\bar{\alpha}}(\Delta, \bar{\Delta}) \sqrt{\bar{E}(\bar{\alpha})}\right\rfloor .
$$

Define $\mathcal{W}$ to be the set of weighted graphs induced by elements of $M_{\mathcal{R}}\left(\Delta, \Delta_{0}, \tau\right)$ :

$$
\mathcal{W}=\left\{\bar{W} \mid \bar{\Delta} \in M_{\mathcal{R}}\left(\Delta, \Delta_{0}, \tau\right)\right\} .
$$

The weighted graph $\bar{W}$ essentially determines $\bar{\Delta}$ except that, for $\bar{\alpha} \in \overline{\mathcal{S}}^{c}$, the value of $m(\bar{\alpha}, \bar{\Delta})$ determines twist $t_{\bar{\alpha}}(\Delta, \bar{\Delta})$ only up to $\frac{1}{\sqrt{\bar{E}(\bar{\alpha})}}=\frac{1}{\sqrt{E_{0}\left(\alpha_{0}\right)}}$ possibilities (we have used the floor function in defining $m(\bar{\alpha}, \bar{\Delta})$ ). Hence,

$$
\left|M_{\mathcal{R}}\left(\Delta, \Delta_{0}, \tau\right)\right| \leq \prod_{\alpha_{0} \in \mathcal{S}_{0}^{c}} \frac{1}{\sqrt{E_{0}\left(\alpha_{0}\right)}}|\mathcal{W}| .
$$

We proceed in two steps:

Step 1 Consider the set $\mathcal{E} \subset U$ that forms a basis for the space $\mathbb{R}[U] /\langle\mathcal{R}\rangle$. First, we claim that the map

$$
\mathcal{I}: \mathcal{W} \rightarrow \mathbb{N}^{h_{\mathcal{R}}}, \quad \bar{W} \rightarrow(\mathrm{i}(\bar{W}, \omega))_{\omega \in \mathcal{E}}
$$

is finite to one, where $\mathrm{i}(\bar{W}, \omega)$ is defined to be

$$
\mathrm{i}(\bar{W}, \omega)=\sum_{\bar{\omega} \in \bar{T}} \mathrm{i}(\bar{\omega}, \omega)+\sum_{\bar{\alpha} \in \overline{\mathcal{S}}^{c}} m(\bar{\alpha}, \bar{\Delta}) \mathrm{i}(\bar{\alpha}, \omega) .
$$

Note that in general a weighted graph $\bar{W}$ is determined by the intersection numbers of its edges with all the edges of $U$. The map $\mathcal{I}$ records the intersection number with arcs in $\mathcal{E}$. To prove the claim, we need to show that, there are only finitely many possibilities for the intersection number of $\bar{W}$ with the other edges of $U$.

We can consider an $v \in U$ as the element $1 \cdot v \in \mathbb{R}[U]$. Then $v$ can be written as a linear combination elements in the generating set $\mathcal{E}$ (which 
generates $\mathbb{R}[U] /\langle\mathcal{R}\rangle)$ ) and $\mathcal{R}$ (the relations). That is, there are constants $c_{\omega}$ and $d_{R}$ so that

$$
v=\sum_{\omega \in \mathcal{E}} c_{\omega} \omega+\sum_{R \in \mathcal{R}} d_{R} R
$$

But the intersection number is linear hence, for every $\bar{\omega} \in \bar{W}$, we have

$$
\mathrm{i}(v, \bar{\omega})=\sum_{\omega \in \mathcal{E}} c_{\omega} \mathrm{i}(\omega, \bar{\omega})+O\left(\sum_{R \in \mathcal{R}} d_{R}\right) .
$$

But the constants $d_{R}$ depend only on the set $\mathcal{R}$ and otherwise are uniformly bounded. Hence, there are only finite number of possibilities for $\mathrm{i}(v, \bar{\omega})$. This proves the claim.

Step 2 We bound the size of $\mathcal{I}(\mathcal{W}) \subset \mathbb{N}^{h}$ by obtaining upper bounds on intersection numbers of $\bar{W}$ with $\operatorname{arcs} \omega \in \mathcal{E}$.

- First, if $\omega \in T$, Eq. (30) implies that

$$
\mathrm{i}(\bar{T}, \omega) \stackrel{*}{\prec} e^{\tau} .
$$

Also, for $\bar{\alpha} \in \overline{\mathcal{S}}^{c}-\mathcal{S}^{c}$ the Eq. (31) implies

$$
m(\bar{\alpha}, \bar{\Delta}) \mathrm{i}(\bar{\alpha}, \omega) \stackrel{*}{\prec} \operatorname{twist}_{\bar{\alpha}}(\Delta, \bar{\Delta}) \sqrt{\bar{E}(\bar{\alpha})} \mathrm{i}(\bar{\alpha}, \omega) \stackrel{*}{\prec} e^{\tau} .
$$

Hence,

$$
\mathrm{i}(\bar{W}, \omega) \stackrel{*}{\prec} e^{\tau} .
$$

- For $\operatorname{arc} \beta_{\alpha} \in U$ where $\alpha \in \mathcal{S}^{c}$, and $\operatorname{arc} \bar{\omega} \in \bar{W}$ by Eq. (32) we have

$$
\mathrm{i}\left(\beta_{\alpha}, \bar{\omega}\right) \stackrel{*}{\prec} \mathrm{i}(\alpha, \bar{\omega}) \operatorname{twist}_{\alpha}(\Delta, \bar{\Delta}) \stackrel{*}{\prec} \frac{e^{\tau}}{\sqrt{E(\alpha)}} .
$$

And for $\bar{\alpha} \in \overline{\mathcal{S}}^{c}-\mathcal{S}^{c}$, by Eq. (33) we have

$$
m(\alpha, \Delta) \mathrm{i}(\alpha, \bar{\alpha}) \stackrel{*}{\prec} \operatorname{twist}_{\bar{\alpha}}(\Delta, \bar{\Delta}) \sqrt{\bar{E}(\bar{\alpha})} \mathrm{i}(\alpha, \bar{\alpha}) \stackrel{*}{\prec} \frac{e^{\tau}}{\sqrt{E(\alpha)}} .
$$

Finally, if $\alpha=\bar{\alpha} \in \mathcal{S}^{c} \cap \overline{\mathcal{S}}^{c}$, by Eq. (34)

$$
m(\bar{\alpha}, \bar{\Delta}) \mathrm{i}\left(\bar{\alpha}, \beta_{\alpha}\right) \stackrel{*}{\prec} \frac{e^{\tau}}{\sqrt{E(\alpha)}} .
$$


Now from Eqs. (37), (38), (39) and (40), we get:

$$
\begin{aligned}
|\mathcal{W}| \stackrel{*}{\prec}|\mathcal{I}(\mathcal{W})| \stackrel{*}{\prec} \prod_{\substack{\alpha \in \mathcal{S}^{c} \\
\beta_{\alpha} \in \mathcal{E}}} \frac{e^{\tau}}{\sqrt{E(\alpha)}} \times \prod_{\omega \in T \cap \mathcal{E}} e^{\tau} \\
\leq e^{|\mathcal{E}| \tau} \prod_{\alpha \in \mathcal{S}^{c}} \frac{1}{\sqrt{E(\alpha)}} .
\end{aligned}
$$

Now, applying Eq. (35), we get

$$
\left|M_{\mathcal{R}}\left(\Delta, \Delta_{0}, \tau\right)\right| \stackrel{*}{\prec} e^{h_{\mathcal{R}} \tau} \prod_{\alpha \in \mathcal{S}^{c}} \frac{1}{\sqrt{E(\alpha)}} \prod_{\alpha_{0} \in \mathcal{S}_{0}^{c}} \frac{1}{\sqrt{E_{0}\left(\alpha_{0}\right)}},
$$

which is as we claimed.

Proof of Theorem 5.1 Let $Z \in B_{j}(\mathcal{Q}(\sigma), X, Y, \tau)$ and let $\left(X_{a}, q_{a}\right)$ and $\left(X_{b}, q_{b}\right)$ be the initial and the terminal quadratic differentials for the Teichmüller geodesic in $\mathcal{Q}(\sigma)$ starting near $X$ and finishing near $Z \in \Gamma \cdot Y$, as before. There may be many choices for these quadratic differentials. We need to be a bit careful.

Claim We can choose $\left(X_{a}, q_{a}\right)$ and $\left(X_{b}, q_{b}\right)$ so that for any $\alpha \in \mathcal{S}_{q_{a}}^{\leq \tau}$,

$$
\text { twist }_{\alpha}\left(X, q_{a}\right)=O(\tau) \text {. }
$$

Proof of claim Assume $\left(X_{\hat{a}}, q_{\hat{a}}\right)$ and $\left(X_{\hat{b}}, q_{\hat{b}}\right)$ are some choice of initial and terminal points with associated regular triangulations $T_{\hat{a}}$ and $T_{\hat{b}}$ that have $j$ common saddle connection. But, assume that they do not satisfy Eq. (41). We define $\left(X_{a}, q_{a}\right)$ to be the image of $\left(X_{\hat{a}}, q_{\hat{a}}\right)$ under an appropriate number of Dehn twists around curves in $\mathcal{S}_{q_{\hat{a}}}^{\leq \tau}$ to ensure (41) and let $\left(X_{b}, q_{b}\right)$ be the image of $\left(X_{\hat{b}}, q_{\hat{b}}\right)$ under the same homeomorphism. We will show that $X_{a}$ and $X_{b}$ are still near $X$ and $Z$.

For $\alpha \in \mathcal{S}_{q_{\hat{a}}}^{\leq \tau}$, if $\frac{1}{\operatorname{Ext}_{X}(\alpha)} \stackrel{*}{\prec} \tau$, by Lemma 3.16.

$$
\operatorname{twist}_{\alpha}\left(X_{\hat{a}}, q_{\hat{a}}\right) \stackrel{*}{\prec} \frac{1}{\operatorname{Ext}_{X}(\alpha)} .
$$

Hence, using the triangle inequality and Theorem 2.2

$$
\begin{aligned}
\operatorname{twist}_{\alpha}\left(X, q_{\hat{a}}\right) & \stackrel{+}{\gtrless} \operatorname{twist}_{\alpha}\left(X, X_{\hat{a}}\right)+\operatorname{twist}_{\alpha}\left(X_{\hat{a}}, q_{\hat{a}}\right)=O\left(\frac{1}{\operatorname{Ext}_{X}(\alpha)}\right) \\
& =O(\tau) .
\end{aligned}
$$


Therefore, (41) is holds and no modification is required.

Now, assume $\frac{1}{\operatorname{Ext}_{X}(\alpha)} \stackrel{*}{\succ} \tau$. Since $\alpha$ is a non-cylinder curve, $\frac{1}{\operatorname{Ext}_{X_{t}}(\alpha)}$ changes at most linearly with time (Eq. (7)). Hence, for $\tau$ large enough, we have

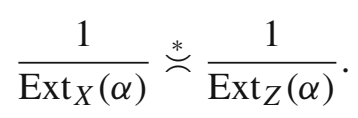

Again by Lemma 3.16, the number of Dehn twists $n_{\alpha}$ around $\alpha$ that needs to be applied to $q_{\hat{a}}$ to ensure Eq. (41) is at most $O\left(1 / \operatorname{Ext}_{X}(\alpha)\right)$. That is,

$$
X_{a}=\prod_{\alpha \in \mathcal{S}_{q_{\hat{a}}}^{\leq \tau}} D_{\alpha}^{n_{\alpha}} X_{q_{0}},
$$

where $D_{\alpha}$ is a Dehn twist around $\alpha$ and $n_{\alpha} \stackrel{*}{\prec} \frac{1}{\operatorname{Ext}_{X}(\alpha)}$. By, Theorem 2.2

$$
d_{\mathcal{T}}\left(X_{\hat{a}}, X_{a}\right) \stackrel{*}{\rightleftharpoons} \sum_{\alpha \in \mathcal{S}_{q_{\hat{a}}}^{\leq \tau}} n_{\alpha} \operatorname{Ext}_{X}(\alpha) \stackrel{*}{\prec} 1 .
$$

and

$$
d_{\mathcal{T}}\left(Y_{\hat{b}}, X_{b}\right) \stackrel{*}{\asymp} \sum_{\alpha \in \mathcal{S}_{q_{\hat{a}}}^{\leq \tau}} n_{\alpha} \operatorname{Ext}_{Z}(\alpha) \stackrel{*}{\prec} 1 .
$$

Hence, $\left(X_{a}, q_{a}\right)$ and $\left(X_{b}, q_{b}\right)$ are as desired. Also, the images $T_{a}$ and $T_{b}$ of $T_{\hat{a}}$ and $T_{\hat{b}}$ are still regular triangulations and have $j$ arcs in common.

For the rest of the proof, we assume Eq. (41) holds. To the pair $\left(X_{a}, q_{a}\right)$ we associate the marking $\Delta=\{\mathcal{S},\{E(\alpha)\}, T\}$ as follows:

- Let $\mathcal{S}$ be the set of short curve in $X$ and set $E(\alpha)=\operatorname{Ext}_{X}(\alpha)$.

- Let $T$ be the $\left(q_{a}, \tau\right)$-regular triangulation $T_{a}$ which has $j$ edges in common with the triangulation $T_{b}$.

- If $\alpha \in \mathcal{S}_{q_{a}}^{\geq \tau}$ then set the twisting around $\alpha$ in $\Delta$ so that

$$
\operatorname{twist}_{\alpha}(\Delta, X)=O(1) \text {. }
$$

- If $\alpha \in \mathcal{S}_{q_{a}}^{\leq \tau}$ then set the twisting around $\alpha$ in $\Delta$ so that

$$
\operatorname{twist}_{\alpha}(\Delta, T)=O(1) \text {. }
$$

The result is a marking that has bounded length in $X$ and [by Eq. (41)] has $\tau$-bounded twist in $X$. Also, note that $\mathcal{S}^{c}=\mathcal{S}_{q_{a}}^{\geq \tau}$ and $\mathcal{S}^{n}=\mathcal{S}_{q_{a}}^{\leq \tau}$. 
We can similarly associate a marking $\bar{\Delta}$ to the pair $\left(X_{b}, q_{b}\right)$. Here we can only conclude that $\bar{\Delta}$ is bounded in $Z$ (not with bounded twist); this is because the inequality (41) does not necessarily hold for $Z$ and $q_{b}$. Instead, similar to Eq. (42), we have

$$
\operatorname{twist}_{\bar{\alpha}}\left(Z, q_{b}\right) \stackrel{*}{\prec} \frac{1}{\operatorname{Ext}_{Z}(\bar{\alpha})} \text {. }
$$

Assume $Z=\mathfrak{g}(Y)$, for $\mathfrak{g} \in \Gamma(S)$. Let $\Delta_{0}=\mathfrak{g}^{-1}(\bar{\Delta})$. Then $\Delta_{0}$ in bounded in $Y$. Also, let $\mathcal{R}$ be the elements in $\mathbb{R}[U]$ coming from Lemma 4.7, (and Lemma 4.8 in case quadratic differentials in $\mathcal{Q}(\sigma)$ are not orientable) and the $j$ edges in $T$ that are present in the $\left(q_{b}, \tau\right)$-regular triangulation $T_{b}$. Taking this $T_{b}$ is the partial triangulation in $\bar{\Delta}$, we have $\bar{\Delta} \in M_{\mathcal{R}}\left(\Delta, \Delta_{0}, \tau\right)$. The number of possible choices for $\Delta$ is $O\left(\tau^{\left|\mathcal{S}_{X}\right|}\right)$ (Lemma 5.6) and there are finitely many choices of for the homeomorphism type of $\Delta_{0}$. Lemma 5.8 provides an upperbound for the size of the set $M_{\mathcal{R}}\left(\Delta, \Delta_{0}, \tau\right)$. Also, using the fact that $\bar{\Delta}$ is bounded in $Z$ and Eq. (44), similar to Lemma 5.6, we can conclude that the association $Z \mapsto \bar{\Delta}$ is at most $O\left(\prod_{\alpha \in \mathcal{S}_{q_{b}}^{\leq \tau}} \frac{1}{\operatorname{Ext}_{Z}(\alpha)}\right)$-to-one.

To summarize, we have defined a map from $B_{j}(\mathcal{Q}(\sigma), X, Y, \tau)$ to the union of sets of markings $M_{\mathcal{R}}\left(\Delta, \Delta_{0}, \tau\right)$, where $\Delta$ is bounded $X$ with $\tau$-bounded twist and $\Delta_{0}$ is bounded in $Y$. The map is not one-to-one but we have a bound on the multiplicity.

The size of $B_{j}(\mathcal{Q}(\sigma), X, Y, \tau)$ is comparable to the product of the following: the number of choices for $\Delta$, the number of choices for the homeomorphism class of $\Delta_{0}$, the maximum multiplicity of the association $Z \mapsto \bar{\Delta}$ and the size of $M_{\mathcal{R}}\left(\Delta, \Delta_{0}, \tau\right)$. That is,

$$
\begin{aligned}
\left|B_{j}(\mathcal{Q}(\sigma), X, Y, \tau)\right| & \stackrel{*}{\prec}|M(X, \tau)| \times O(1) \times \prod_{\alpha \in \mathcal{S}_{q_{b}}^{\leq \tau}} \frac{1}{\operatorname{Ext}_{Z}(\alpha)} \times\left|M_{\mathcal{R}}\left(\Delta, \Delta_{0}, \tau\right)\right| \\
& * * \tau^{\left|\mathcal{S}_{X}\right|} \prod_{\alpha \in \mathcal{S}_{q b}^{\leq \tau}} \frac{1}{\operatorname{Ext}_{Z}(\alpha)} e^{h_{\mathcal{R}} \tau} \prod_{\alpha \in \mathcal{S}^{c}} \frac{1}{\sqrt{E(\alpha)}} \prod_{\alpha_{0} \in \mathcal{S}_{0}^{c}} \frac{1}{\sqrt{E_{0}\left(\alpha_{0}\right)}} \\
& * * \tau^{\left|\mathcal{S}_{X}\right|+\left|\mathcal{S}_{Y}\right|} e^{h_{\mathcal{R}} \tau} \prod_{\alpha \in \mathcal{S}} \frac{1}{\sqrt{E(\alpha)}} \prod_{\alpha_{0} \in \mathcal{S}_{0}} \frac{1}{\sqrt{E_{0}\left(\alpha_{0}\right)}} .
\end{aligned}
$$

The last line follows from the previous line because, for every term in the product $\prod_{\alpha \in \mathcal{S}_{q_{b}}^{\leq \tau}} \frac{1}{\operatorname{Ext}_{Z}(\alpha)}$ we either have $\frac{1}{\operatorname{Ext}_{Z}(\alpha)}=O(\tau)$ or, as in Eq. (43),

$$
\frac{1}{\operatorname{Ext}_{Z}(\alpha)} \stackrel{*}{\prec} \frac{1}{\sqrt{\operatorname{Ext}_{Z}(\alpha)}} \frac{1}{\sqrt{\operatorname{Ext}_{X}(\alpha)}}, \quad \text { and } \quad \alpha \in \mathcal{S}_{q_{a}}^{\leq \tau} \cap \mathcal{S}_{q_{b}}^{\leq \tau}=\mathcal{S}^{n} \cap \mathcal{S}_{0}^{n}
$$


That is, each term can either be counted in the power of $\tau$ in the beginning of last line or it can be divided into a term in each of the last two products. The proof is finished after checking that $h_{\mathcal{R}}=(h-j)$. This is true because all the relations in Lemma 4.7 are also relations in $H_{1}(S, \Sigma)$. The fact that the $j$ arcs we have fixed in $T_{a}$ are homologically independent implies that these arcs and the other relations in homology are independent in $\mathbb{R}[U]$. In fact, Lemma 4.8 is used only when $\varsigma=-1$. But this is accounted for in the definition of $h$ (see Sect. 2.9). Hence, the dimension of $\mathbb{R}[U] /\langle\mathcal{R}\rangle$ is exactly $j$ less than $h=\frac{\operatorname{dim} \mathcal{C}+1}{2}$.

\section{Geodesics in the thin part of moduli space}

In this section we prove Theorem 1.5 and Theorem 1.5. The main idea, which is due to Margulis, is to prove an inequality, which shows that the flow (or more precisely an associated random walk) is biased toward a compact part of the space. Consider the stratum $\mathcal{Q}(\sigma)$. We discretize the projection

$$
\pi(\mathcal{Q}(\sigma)) \subset \mathcal{T}(S)
$$

by fixing an appropriate net $\tilde{\mathcal{N}}$ in $\mathcal{T}(S)$. Then, we consider the random walk $\left\{\lambda_{i}\right\}_{i \geq 0}$ on the points in $\widetilde{\mathcal{N}}$ and apply Theorem 5.1 to show that the projection of this random walk in $\mathcal{M}(S)$ is biased towards the compact subset of $\mathcal{M}(S)$. Moreover, we show that quadratic differentials $\left\{q\left(\lambda_{i}, \lambda_{i+1}\right)\right\}_{i \geq 0}$ (see Sect. 2.2) tend not to have short saddle connections. See Lemma 6.4 for the precise formulation.

These estimates imply Theorem 1.5; this is because, roughly speaking, every closed geodesic in $\mathcal{C}$ can be approximated by a path along the net points.

\subsection{Short saddle connections and simple closed curves}

For a quadratic differential $(X, q) \in \mathcal{Q}^{1} \mathcal{T}(S)$, recall the set of short saddle connections $\Omega_{q}(\epsilon)$ (Definition 3.8). Define $s(q, \epsilon)$ to denote the maximum number of homologically independent disjoint saddle connections in $\Omega_{q}(\epsilon)$. Given the tuple $\sigma$, define

$$
\mathcal{Q}_{j, \epsilon}(\sigma)=\{(X, q) \in \mathcal{Q}(\sigma) \mid s(q, \epsilon) \geq j\} \subset \mathcal{Q}^{1} \mathcal{T}(S) .
$$

For the rest of this section, with fix $\sigma$ and denote $\mathcal{Q}_{j, \epsilon}(\sigma)$ simply by $\mathcal{Q}_{j, \epsilon}$. Also, recall the definition of $B(\mathcal{Q}(\sigma), X, \tau)$ from Sect. 4.1 and $B_{j}(\mathcal{Q}(\sigma), X, \tau)$ from Sect.5. We would like to refine the definition of $B_{j}(\mathcal{Q}(\sigma), X, \tau)$. Roughly speaking, we are interested in a ball of radius $\tau$ centered at $X$ that is allowed 
to move in the direction $\mathcal{Q}_{j, \epsilon}$ only. Namely, define $B\left(\mathcal{Q}_{j, \epsilon}, X, \tau\right)$ to be the set $Z \in \mathcal{T}(S)$ so that

- $Z \in B(\mathcal{Q}(\sigma), X, \tau)$

- for the associated quadratic differential $q_{a}$, we have $s\left(q_{a}, \epsilon\right) \geq j$.

One can similarly define $B\left(\mathcal{Q}_{j, \epsilon}, X, Y, \tau\right)$ as in Sect.5. Recall the choice of $\epsilon_{1}(\tau)$ from Lemma 3.13.

Lemma 6.1 For any $\tau>0$, there is $\epsilon_{2}(\tau)<\epsilon_{1}(\tau)$ such that for $\epsilon<\epsilon_{2}(\tau)$, any integer $j \geq 0$, and any $X, Y \in \mathcal{T}(S)$, we have

$$
B\left(\mathcal{Q}_{j, \epsilon}, X, \tau\right) \subset B_{j}(\mathcal{Q}(\sigma), X, \tau),
$$

and

$$
B\left(\mathcal{Q}_{j, \epsilon}, X, Y, \tau\right) \subset B_{j}(\mathcal{Q}(\sigma), X, Y, \tau) .
$$

Proof It is enough to let $\epsilon_{2}(\tau)=e^{-2 \tau} \epsilon_{1}$. Assume, $Z \in B\left(\mathcal{Q}_{j, \epsilon}, X, \tau\right), q_{a}$ and $q_{b}$ are the associated quadratic differentials and $(b-a)<\tau$. Let $\omega_{1}, \ldots, \omega_{j}$ be disjoint homologically independent saddle connections counted in $s\left(q_{a}, \epsilon\right)$. Then, for each $i$, by Eq. (8),

$$
\left|\frac{1}{\operatorname{Ext}_{q_{a}}\left(\omega_{i}\right)}-\frac{1}{\operatorname{Ext}_{q_{b}}\left(\omega_{i}\right)}\right| \leq \tau,
$$

and by Theorem 2.1 the extremal length of any short curve $\alpha$ containing $\omega_{i}$ changes by at most a factor of at most $e^{2 \tau}$. That is, $\omega_{i} \in \Omega_{q_{b}}\left(\epsilon_{1}\right)$. The arcs $\omega_{i}$ are still disjoint and homologically independent in $q_{b}$. Hence, the set $\left\{\omega_{i}\right\}$ can be extended to both a $\left(q_{a}, \tau\right)$-regular triangulation $T_{a}$ and a $\left(q_{b}, \tau\right)$-regular triangulations $T_{b}$ (Lemma 3.13). Thus, by the definition $Z \in B_{j}(\mathcal{Q}(\sigma), X, \tau)$. The proof of Eq. (46) is similar.

\subsection{Choosing a net}

By a $(c, 2 c)$-separated net $\mathcal{N} \subset \mathcal{M}(S)$ we mean a set of points in $\mathcal{M}(S)$ so that:

- the Teichmüller distance between any two net points in $\mathcal{N}$ is at least $c$, and

- any point in $\mathcal{M}(S)$ is within distance $2 c$ of a point in $\mathcal{N}$.

Let

$$
\mathcal{N}(X, \tau)=\mathbf{p}(B(X, \tau)) \cap \mathcal{N} .
$$

Then, it is easy to check (see Lemma 3 in [16]): 
Lemma 6.2 There exists a constant $c_{0}>0$ such that for any $c>c_{0}$, and $(c, 2 c)$ net $\tilde{\mathcal{N}}$ as above, we have

$$
|\mathcal{N}(X, \tau)| \stackrel{*}{\prec} \tau^{3 g-3+p} .
$$

Let $\tilde{\mathcal{N}}=\mathbf{p}^{-1}(\mathcal{N})$. We assume the $r_{0}>2 c$, where $r_{0}$ is the constant used to define $B(\mathcal{Q}(\sigma), X, \tau)$ (see Sect. 4.1). We denote the intersection of a ball in Teichmüller space, $B(\cdot)$, with $\widetilde{\mathcal{N}}$ by $\widetilde{\mathcal{N}}(\cdot)$. That is, for $X, Y \in \mathcal{T}(S)$,

$$
\begin{aligned}
\tilde{\mathcal{N}}(\mathcal{Q}(\sigma), X, \tau) & =B(\mathcal{Q}(\sigma), X, \tau) \cap \tilde{\mathcal{N}}, \\
\tilde{\mathcal{N}}\left(\mathcal{Q}_{j, \epsilon}, X, \tau\right) & =B\left(\mathcal{Q}_{j, \epsilon}, X, \tau\right) \cap \tilde{\mathcal{N}}, \\
\tilde{\mathcal{N}}(\mathcal{Q}(\sigma), X, Y, \tau) & =B(\mathcal{Q}(\sigma), X, Y, \tau) \cap \tilde{\mathcal{N}}, \\
\text { and } \tilde{\mathcal{N}}\left(\mathcal{Q}_{j, \epsilon}, X, Y, \tau\right) & =B\left(\mathcal{Q}_{j, \epsilon}, X, Y, \tau\right) \cap \tilde{\mathcal{N}}
\end{aligned}
$$

\subsection{The main inequality}

For a real-valued function $f: \mathcal{M}(S) \rightarrow \mathbb{R}$, consider the average function

$$
\left(A_{j, \epsilon}^{\tau} f\right): \mathcal{T}(S) \rightarrow \mathbb{R}
$$

defined by

$$
\left(A_{j, \epsilon}^{\tau} f\right)(X)=e^{-h \tau} \sum_{Z \in \tilde{\mathcal{N}}\left(\mathcal{Q}_{j, \epsilon}, X, \tau\right)} f(Z) .
$$

Here, as before

$$
h=\frac{\operatorname{dim} \mathcal{C}+1}{2}
$$

Our main tool is the following $\left(\epsilon_{2}(\tau)\right.$ is as in Lemma 6.1):

Proposition 6.3 Given $\tau>0$, and $\epsilon<\epsilon_{2}(\tau)$ we have

$$
\left(A_{j, \epsilon}^{\tau} G\right)(X) \stackrel{*}{\prec} \tau^{m} e^{-j \tau} G(X) .
$$

where $G$ is as in Eq. (4) and $m$ depends only on the topology of $S$.

Proof Enumerate the elements of $\mathcal{N}(X, \tau)$ as $y_{1}, \ldots, y_{k}$ and let $Y_{i} \in \mathcal{T}(S)$ be a pre-image of $y_{i}, i=1, \ldots, k$. By Lemma 2.3 , every net point in $Z \in$ 
$B\left(\mathcal{Q}_{j, \epsilon}, \tilde{\mathcal{N}}, X, Y_{i}, \tau\right)$ is near at most $G\left(Y_{i}\right)$ points in $B\left(\mathcal{Q}_{j, \epsilon}, X, Y_{i}, \tau\right)$. That is,

$$
\left|\tilde{\mathcal{N}}\left(\mathcal{Q}_{j, \epsilon}, X, Y_{i}, \tau\right)\right| G\left(Y_{i}\right)^{2} \stackrel{*}{\asymp}\left|B\left(\mathcal{Q}_{j, \epsilon}, X, Y_{i}, \tau\right)\right|
$$

Hence, we have

$$
\begin{gathered}
\left(A_{j, \epsilon}^{\tau} G\right)(X)=e^{-h \tau} \sum_{Z \in \tilde{\mathcal{N}}\left(\mathcal{Q}_{j, \epsilon}, X, \tau\right)} \sum_{i=1} \sum_{Z \in \widetilde{\mathcal{N}}\left(\mathcal{Q}_{j, c}, k\right.} \sum^{-h \tau} \sum_{i=1}^{*} \frac{\left|B\left(\mathcal{Q}_{j, \epsilon}, X, Y_{i}, \tau\right)\right|}{G\left(Y_{i}\right)} \\
\stackrel{*}{\gtrless} e^{-h \tau} \sum_{i=1}^{k} \tau^{\left|\mathcal{S}_{X}\right|+\left|\mathcal{S}_{Y}\right|} e^{(h-j) \tau} G(X) \\
\leq e^{-j \tau} \tau^{m} G(X) .
\end{gathered}
$$

Here, $m=(9 g-9+3 p) \geq\left|\mathcal{S}_{X}\right|+\left|\mathcal{S}_{Y}\right|+(3 g-3+p)$.

Trajectories of the random walk Suppose $R>>\tau$ and let $n$ be the integer part of $R / \tau$. By a trajectory of the random walk we mean a map

$$
\lambda:\{0, n\} \rightarrow \tilde{\mathcal{N}} \subset \mathcal{T}(S)
$$

such that, for all $0<k \leq n$, we have $d_{\mathcal{T}}\left(\lambda_{k}, \lambda_{k-1}\right) \leq \tau$, where $\lambda_{k}=\lambda(k)$. Let $\mathcal{P}_{\tau}(X, R)$ denote the set of all trajectories for which $d_{\mathcal{T}}\left(\lambda_{0}, X\right)=O(1)$. For $j \in \mathbb{N}$, let $\mathcal{P}_{\theta, \tau}\left(\mathcal{Q}_{j, \epsilon}, X, R\right)$ denote the set of all trajectories $\lambda \in \mathcal{P}_{\tau}(X, R)$ so that,

- for $1 \leq k \leq n$

$$
\lambda_{k} \in \tilde{\mathcal{N}}\left(\mathcal{Q}(\sigma), \lambda_{k-1}, \tau\right)
$$




$$
\left|\left\{k \mid 1 \leq k \leq n, \lambda_{k} \in B\left(\mathcal{Q}_{j, \epsilon}, \lambda_{k-1}, \tilde{\mathcal{N}}, \tau\right)\right\}\right| \geq \theta \cdot n .
$$

Given $X, Y \in \mathcal{T}$, let $\mathcal{P}_{\theta, \tau}\left(\mathcal{Q}_{j, \epsilon}, X, Y, R\right)$ denote the set of all trajectories $\lambda \in \mathcal{P}_{\theta, \tau}\left(\mathcal{Q}_{j, \epsilon}, X, R\right)$ such that

$$
d_{\mathcal{T}}\left(\mathbf{p}(Y), \mathbf{p}\left(\lambda_{n}\right)\right)=O(1) .
$$

We say that a trajectory is almost closed in the quotient if

$$
d_{\mathcal{T}}\left(\mathbf{p}\left(\lambda_{0}\right), \mathbf{p}\left(\lambda_{n}\right)\right)=O(1) .
$$

Finally, let $\widehat{\mathcal{P}}_{\theta, \tau}\left(\mathcal{Q}_{j, \epsilon}, X, R\right)=\mathcal{P}_{\theta, \tau}\left(\mathcal{Q}_{j, \epsilon}, X, X, R\right)$ denote the subset of these trajectories starting from $X$ which are almost closed in the quotient. Let $\epsilon_{2}(\tau)$ be as in Lemma 6.1 and Proposition 6.3.

Lemma 6.4 For any $\delta_{0}>0$ there is $\tau_{0}>0$ so that for $\tau>\tau_{0}, 0 \leq \theta \leq 1$ and $\epsilon<\epsilon_{2}\left(\tau_{0}\right)$ we have

$$
\left|\mathcal{P}_{\theta, \tau}\left(\mathcal{Q}_{j, \epsilon}, X, Y, R\right)\right| \stackrel{*}{\prec} e^{\left(h-j \theta+\delta_{0}\right) R} \frac{G(X)}{G(Y)} .
$$

In particular,

$$
\left|\widehat{\mathcal{P}}_{\theta, \tau}\left(\mathcal{Q}_{j, \epsilon}, X, R\right)\right| \stackrel{*}{\prec} e^{\left(h-j \theta+\delta_{0}\right) R} .
$$

Proof Define

$$
q_{k}(\lambda)=\left|\left\{i \mid 1 \leq i \leq k, \lambda_{i} \in \tilde{\mathcal{N}}\left(\mathcal{Q}_{j, \epsilon}, \lambda_{i-1}, \tau\right)\right\}\right| .
$$

This keeps track of the number of steps in the trajectory $\lambda$ (amount the first $k$ steps) that can be approximated by a segment in $\mathcal{Q}_{j, \epsilon}$. For $0<r=k \tau<R$, let $\widehat{\mathcal{P}}_{\theta, \tau}\left(\mathcal{Q}_{j, \epsilon}, X, Y, R, r\right)$ be the set of trajectories obtained from a trajectory $\lambda \in \widehat{\mathcal{P}}_{\theta, \tau}\left(\mathcal{Q}_{j, \epsilon}, X, Y, R\right)$ but truncated after $k=r / \tau$ steps. Define

$$
V_{\tau}(R, r)=\sum_{\lambda \in \mathcal{P}_{\theta, \tau}\left(\mathcal{Q}_{j, \epsilon}, X, Y, R, r\right)} G\left(\lambda_{k}\right) e^{j q_{k}(\lambda) \tau} .
$$

Also, let $R=n \tau, q(\lambda)=q_{n}(\lambda)$ and

$$
V_{\tau}(R)=\sum_{\lambda \in \mathcal{P}_{\theta, \tau}\left(\mathcal{Q}_{j, \epsilon}, X, Y, R\right)} G\left(\lambda_{n}\right) e^{j q(\lambda) \tau} .
$$


Note that $G(Y) \stackrel{*}{\asymp} G\left(\lambda_{n}\right)$ and $q(\lambda) \tau \geq \theta R$. Therefore,

$$
\left|\mathcal{P}_{\theta, \tau}\left(\mathcal{Q}_{j, \epsilon}, X, Y, R\right)\right| \stackrel{*}{\prec} \frac{V_{\tau}(R)}{G(Y) e^{j \theta R}} .
$$

If $\lambda_{k+1} \in \tilde{\mathcal{N}}\left(\mathcal{Q}_{j, \epsilon}, \lambda_{k}, \tau\right)$ then $q_{k+1}(\lambda)=q_{k}(\lambda)+1$ and $q_{k+1}(\lambda)=q_{k}(\lambda)$ otherwise. Hence,

$$
\begin{aligned}
V_{\tau}(R, r+\tau)= & \sum_{\lambda \in \mathcal{P}_{\theta, \tau}\left(\mathcal{Q}_{j, \epsilon}, X, Y, R, r+\tau\right)} G\left(\lambda_{k+1}\right) e^{j q_{k+1}(\lambda) \tau} \\
\leq & \sum_{\lambda \in \mathcal{P}_{\theta, \tau}\left(\mathcal{Q}_{j, \epsilon}, X, Y, R, r\right)}\left(\sum_{\lambda_{k+1} \in \tilde{\mathcal{N}}\left(\mathcal{Q}_{j, \epsilon}, \lambda_{k}, \tau\right)} G\left(\lambda_{k+1}\right) e^{j\left(q_{k}(\lambda)+1\right) \tau}+\right. \\
& \left.+\sum_{\lambda_{k+1} \notin \tilde{\mathcal{N}}\left(\mathcal{Q}_{j, \epsilon}, \lambda_{k}, \tau\right)} G\left(\lambda_{k+1}\right) e^{\left.j q_{k}(\lambda) \tau\right)}\right)
\end{aligned}
$$

The two summands inside of the parenthesis are similar to the average defined above. Using Eq. (48), the first term is less than (up to a multiplicative error)

$$
e^{j\left(q_{k}(\lambda)+1\right) \tau} e^{h \tau}\left(A_{j, \epsilon}^{\tau} G\right)\left(\lambda_{k}\right) \stackrel{*}{\prec} e^{j\left(q_{k}(\lambda)+1\right) \tau} e^{h \tau} \tau^{m} e^{-j \tau} G\left(\lambda_{k}\right) .
$$

and the second term is less than (again, up to a multiplicative error)

$$
e^{j q_{k}(\lambda) \tau} e^{h \tau}\left(A_{\tau, 0} G\right)\left(\lambda_{k}\right) \stackrel{*}{\prec} e^{j q_{k}(\lambda) \tau} e^{h \tau} \tau^{m} G\left(\lambda_{k}\right) \text {. }
$$

Note that the right hand sides of the above two equations are the same. Hence,

$$
\begin{aligned}
V_{\tau}(R, r+\tau) & \stackrel{*}{\prec} \tau^{m} e^{h \tau} \sum_{\lambda \in \mathcal{P}_{\tau}(\mathcal{C}, X, R, r)} e^{j q_{k}(\lambda) \tau} G\left(\lambda_{k}\right) \\
& =\tau^{m} e^{h \tau} V_{\tau}(R, r) .
\end{aligned}
$$

Now iterating (53) $n=R / \tau$ times we get

$$
V_{\tau}(R) \leq(C \tau)^{m n} G(X) e^{h n \tau}=G(X) e^{\left(h+\frac{m(\log (\tau)+\log (C))}{\tau}\right) R},
$$

where $C>0$, and $m \in \mathbb{N}$ are uniform constants. We choose $\tau$ large enough so that

$$
\frac{m \log (\tau)+\log (C)}{\tau}<\delta_{0}
$$


The lemma follows from Eq. (52) and Eq. (54).

Let $N_{\theta}\left(\mathcal{Q}_{j, \epsilon}, X, Y, R\right)$ be the number points $Z \in B(\mathcal{Q}(\sigma), X, Y, R)$ (see Sect. 5 for definition) so that associated geodesic $\left(X_{t}, q_{t}\right)$ spends $\theta$ proportion of time in $\mathcal{Q}_{j, \epsilon}$. Similarly, for $x \in \mathcal{M}(S)$, let $N_{\theta}\left(\mathcal{C}_{j, \epsilon}, x, R\right)$ be the number of conjugacy classes mapping classes associated to closed geodesics $\mathbf{g}$ in $\mathcal{C}$ of length at most $R$ which pass within a uniformly bounded distance of the point $x$ and so that for at least $\theta$ fraction of the points $\left(x_{t}, q_{t}\right) \in \mathbf{g}, s\left(q_{t}, \delta\right) \geq j$ (see Sect.6.1). As we shall see in the proof of the lemma below, for $x=\mathbf{p}(X)$, $N_{\theta}\left(\mathcal{Q}_{j, \epsilon}, X, X, R\right)$ may be much larger than $N_{\theta}\left(\mathcal{C}_{j, \epsilon}, x, R\right)$.

Lemma 6.5 For any $\delta_{1}>0$, there is $\tau_{1}$ so that, for $\tau>\tau_{1} X \in \mathcal{T}(S)$ and any sufficiently large $R$ (depending only on $\delta_{1}, \tau$ ) we have

$$
N_{\theta}\left(\mathcal{C}_{j, \epsilon}, \mathbf{p}(X),\left(1-\delta_{1}\right) R\right) \stackrel{*}{\prec}\left|\mathcal{P}_{\theta, \tau}\left(\mathcal{Q}_{j, \epsilon}, X, R\right)\right|,
$$

and

$$
N_{\theta}\left(\mathcal{Q}_{j, \epsilon}, X, Y,\left(1-\delta_{1}\right) R\right) \stackrel{*}{\prec}\left|\mathcal{P}_{\theta, \tau}\left(\mathcal{Q}_{j, \epsilon}, X, Y, R\right)\right| G(Y)^{2} .
$$

Proof Recall the definition of

$$
I_{X}=\left\{\mathfrak{g} \in \Gamma(S) \mid d_{\mathcal{T}}(X, \mathfrak{g} \cdot X)=O(1)\right\} .
$$

from Lemma 2.3. Consider a closed geodesic $\mathbf{g}$ in $\mathcal{C}$ which intersects a uniformly bounded neighborhood of $x=\mathbf{p}(X)$. Let $[\mathbf{g}]$ denote the corresponding conjugacy class in $\Gamma(S)$. Then there are approximately $\left|I_{X}\right|$ lifts of $[\mathbf{g}]$ to $\mathcal{T}_{g}$ which start within a bounded distance of $X$. Each lift $\mathcal{G}$ is a geodesic segment of length equal to the length of $\mathbf{g}$.

We can mark points distance $\tau$ apart on $\mathcal{G}$, and replace these points by the nearest net points in $\widetilde{\mathcal{N}}$. (This replacement is the cause of the $\delta_{1} R$ error). This gives a map $\Psi$ from lifts of geodesics to trajectories. If the original geodesic $\mathbf{g}$ has length at most $\left(1-\delta_{1}\right) R$ and has $s\left(q_{t}, \delta\right) \geq j$ for $\theta$ fraction of its points, then the resulting trajectory belongs to $\mathcal{P}_{\theta, \tau}\left(\mathcal{Q}_{j, \epsilon}, X, R\right)$.

If two geodesic segments map to the same trajectory, then the segments fellow travel within $O(1)$ of each other. In particular if $\mathfrak{g}_{1}$ and $\mathfrak{g}_{2}$ are the pseudoAnosov elements corresponding to the two geodesics, then $d_{\mathcal{T}}\left(\mathfrak{g}_{2}^{-1} \mathfrak{g}_{1} X, X\right)=$ $O(1)$, thus $\mathfrak{g}_{2}^{-1} \mathfrak{g}_{1} \in I_{X}$.

We now consider all possible geodesics contributing to $N_{\theta}\left(\mathcal{C}_{j, \epsilon}, x,(1-\right.$ $\left.\delta_{1}\right) R$ ); for each of these we consider all the possible lifts which pass near $X$, and then for each lift consider the associated random walk trajectory. We get:

$$
\left|I_{X}\right| N_{\theta}\left(\mathcal{C}_{j, \epsilon}, x,\left(1-\delta_{1}\right) R\right) \stackrel{*}{\prec}\left|I_{X}\right|\left|\mathcal{P}_{\theta, \tau}\left(\mathcal{Q}_{j, \epsilon}, X, R\right)\right| .
$$


The factor of $\left|I_{X}\right|$ on the left hand side is due to the fact that we are considering all possible lifts which pass near $X$, and the factor of $\left|I_{X}\right|$ on the right is the maximum possible number of times a given random walk trajectory can occur as a result of this process. Thus, the factors of $\left|I_{X}\right|$ cancel, and the lemma follows. Note that by Lemma 2.3 (see also, Theorem 5.1) $|I(Y)| \stackrel{*}{\prec} G(Y)^{2}$. An argument similar to the proof of the first part implies Eq. (56).

We need he following lemma which is due to Veech [48].

Lemma 6.6 Suppose $\mathbf{g}$ is a closed geodesic of length at most $R$ on $\mathcal{M}(S)$. Then for any $x \in \mathbf{g}$, any $X$ so that $\mathbf{p}(X)=x$ and every simple closed curve $\alpha$

$$
\operatorname{Ext}_{X}(\alpha) \stackrel{*}{\succ} e^{-(6 g-4+2 p) R} .
$$

Proof of Theorem 1.5 Let $\delta>0$. Choose $\delta_{0}, \delta_{1} \leq \delta / 3$. Now choose $\tau \geq$ $\max \left\{\tau_{0}, \tau_{1}\right\}$ and let $R$ be large enough so that Eqs. (51) and (55) hold. We get,

$$
N_{\theta}\left(\mathcal{C}_{j, \epsilon}, x, R\right) \stackrel{*}{\prec} e^{(h-j \theta+2 \delta / 3) R} .
$$

Finally

$$
N_{\theta}\left(\mathcal{C}_{j, \epsilon} R\right) \leq \sum_{x \in \mathcal{N}} N_{\theta}\left(\mathcal{Q}_{j, \epsilon}, x, R\right)
$$

where $\mathcal{N}$ is the net chosen above. In view of Lemma 6.6 and Lemma 6.2, the number of relevant points in the net is at most polynomial in $R$. However, for $R$ large enough, this polynomial is less than $e^{\delta R / 3}$. Thus Theorem 1.5 follows.

Proof of Theorem 1.5 Let $\mathbf{g}$ be a closed geodesic in $\mathcal{C} \backslash \mathcal{K}$. By taking $\mathcal{K}$ large enough we can assure that every quadratic differential along $\mathbf{g}$ has an arbitrary short saddle connection. We choose $\mathcal{K}$ so that Lemma 3.9 implies that any such quadratic differential $(x, q), \Omega_{q}(\epsilon)$ is non-empty for $\epsilon \leq \epsilon_{2}(\tau)$. Hence the number of disjoint homologically independent saddle connections in $\Omega_{q}(\epsilon)$ is at least one. That is, $\mathbf{g}$ is counted in $N_{\theta}\left(\mathcal{C}_{j, \epsilon}, R\right)$ for $j=1$ and $\theta=1$. The theorem now follows from Theorem 1.5.

Proof We can use the argument applied in the proof of Theorem 1.5. Let $0<\delta_{0}, \delta_{1} \leq \delta / 3$. Choose a net satisfying Lemma 6.2. Then choose $\tau \geq$ $\max \left\{\tau_{0}, \tau_{1}\right\}$ and let $R$ be large enough so that Eqs. (50) and (56) hold. As in the proof of Theorem 1.5, Eq. (1) follows from Lemma 6.2 and Lemma 6.6. 


\section{The Hodge norm and the Hodge distance}

In this section, we use the Hodge norm [19] to show that in any compact subset of $\mathcal{C}$ the geodesic flow is uniformly hyperbolic: see [5] and Remark 7.5 below. There are many approaches to proving hyperbolic like behavior for the Teichmüller geodesic flow in different settings, see for example $[7,8,19,22,48]$.

Let $\mathcal{H}^{1} \mathcal{T}(S)$ be the bundle of area one abelian differentials over $\mathcal{T}(S)$. We also denote by $g_{t}$ the geodesic flow on $\mathcal{H}^{1} \mathcal{T}(S)$ (where we square an abelian differential to get a quadratic differential).

\subsection{Hodge norm}

Fix a point $(X, \phi)$ in $\mathcal{H}^{1} \mathcal{T}(S)$, where $X \in \mathcal{T}(S)$ and $\phi$ is an abelian differential on $X$. Let $\pi: \mathcal{H}^{1} \mathcal{T}(S) \rightarrow \mathcal{T}(S)$ and $\mathbf{p}: \mathcal{H}^{1} \mathcal{T}(S) \rightarrow \mathcal{H}^{1} \mathcal{M}(S)$ be natural maps as in Sect.2.3. Let $\|\cdot\|_{H, t}$ denote the Hodge norm on the surface $X_{t}=$ $\pi\left(g_{t} \phi\right)$. Also, for each abelian differential $\phi$, let $\Re(\phi), \Im(\phi) \in H^{1}(X, R)$ be forms obtained by the real part and the imaginary part of the holonomy.

The following fundamental result is due to Forni $[19, \S 2]$ :

Theorem 7.1 For any $\lambda \in H^{1}(X, \mathbb{R})$ and any $t \geq 0$,

$$
\|\lambda\|_{H, t} \leq e^{t}\|\lambda\|_{H, 0}
$$

If, in addition, $\lambda \wedge \Re(\phi)=\lambda \wedge \Im(\phi)=0$ and, for some compact subset $\mathcal{K}$ of $\mathcal{H}^{1} \mathcal{M}(S)$, the segment $\left[\phi, g_{t} \phi\right]$ starts and ends in $\mathbf{p}^{-1}(\mathcal{K})$ and spends at least half the time in $\mathbf{p}^{-1}(\mathcal{K})$, then we have

$$
\|\lambda\|_{H, t} \leq e^{(1-\alpha) t}\|\lambda\|_{H, 0},
$$

where $\alpha>0$ depends only on $\mathcal{K}$.

Theorem 7.1 gives a partial hyperbolicity property of the geodesic flow on spaces of abelian differentials. In our application, we need a similar property for compact subsets of the spaces $\mathcal{Q}^{1} \mathcal{M}(\sigma)$ of quadratic differentials.

\subsection{Quadratic and abelian differentials}

Here, we briefly treat the case when $q \in \mathcal{Q M}(S)$ is not the global square of an abelian differential. A standard construction, given $X \in \mathcal{T}(S)$ and $q$ a quadratic differential on $X$, is to pass to the possibly ramified double cover on which the foliation defined by $q$ is orientable. More precisely, we consider the canonical (ramified) double cover $\phi: \tilde{X} \rightarrow X$ such that $\phi^{*}(q)=\phi^{2}$. (See the 
proof of Lemma 4.8 for the explicit construction.) The set of critical values of $\phi$ coincides with the set of zeros of $q$ with odd degree.

This yields a surface $\tilde{X}$ with an abelian differential $\phi$. However, even if $\mathbf{p}(X)$ belongs to a compact subset of $\mathcal{M}(S)$, there may be a curve that has a very small extremal length in $\tilde{X}$. This may occur since the flat structure defined by $q$ may have an arbitrarily short saddle connection connecting distinct zeroes. Such a saddle connection lifts to a very short loop in the double cover. Let $\ell_{\min }(q)$ denote the length of the shortest saddle connection in the flat metric defined by $q$. We have,

$$
\ell_{\min }(\phi) \geq \ell_{\min }(q)
$$

That is, if $q$ does not have any short saddle connection, then $\phi$ also does not have any short saddle connections.

\subsection{The Hodge norm on relative cohomology}

Let $(X, q) \in \mathcal{Q}^{1} \mathcal{T}(\sigma)$ and let $\Sigma$ be the set of singularities of $q$. Let $\tilde{X}$ be as before and $\tilde{\Sigma}$ be the pre-image of $\Sigma$. On $\tilde{X}, \tilde{q}$ has a canonical square root which we denote by $\phi$. To simplify the notation, if $q$ is a square of an abelian differential, let $\tilde{X}=X, \tilde{\Sigma}=\Sigma$.

Let $\mathbf{j}: H^{1}(\tilde{X}, \tilde{\Sigma}, \mathbb{R}) \rightarrow H^{1}(\tilde{X}, \mathbb{R})$ denote the natural map. We define a norm $\|\cdot\|$ on the relative cohomology group $H^{1}(\tilde{X}, \tilde{\Sigma}, \mathbb{R})$ as follows:

$$
\|\lambda\|=\|\mathbf{j}(\lambda)\|_{H}+\sum_{\left(p, p^{\prime}\right) \in \Sigma \times \Sigma}\left|\int_{\gamma_{p, p^{\prime}}}(\lambda-\mathfrak{h})\right|,
$$

where $\|\cdot\|_{H}$ denotes the Hodge norm on $H^{1}(\tilde{X}, \mathbb{R}), \mathfrak{h}$ is the harmonic representative of the cohomology class $\mathbf{j}(\lambda)$ and $\gamma_{p, p^{\prime}}$ is any path connecting the zeroes $p$ and $p^{\prime}$. Since $\mathbf{j}(\lambda)$ and $\mathfrak{h}$ represent the same class in $H^{1}(\tilde{X}, \mathbb{R})$, the Eq. (58) does not depend on the choice of $\gamma_{p, p^{\prime}}$.

Let $q_{t}, X_{t}$ and $\phi_{t}$ be defined as usual and let $\|\cdot\|_{t}$ denote the norm (58) on the surface $\tilde{X}_{t}=\pi\left(g_{t} \phi\right)$. We have the following analogue of Theorem 7.1:

Theorem 7.2 Let $\mathcal{K}$ be a compact subset $\mathcal{Q}^{1} \mathcal{M}(\sigma)$. Then there is $t_{0}>0$ so that for $t>t_{0}$ the following holds. Suppose $\mathbf{p}\left(q_{0}\right), \mathbf{p}\left(q_{t}\right) \in \mathcal{K}$ and that the geodesic segment $\left[q_{0}, q_{t}\right]$ spends at least half the time in $\mathbf{p}^{-1}(\mathcal{K})$. Suppose $\lambda \in H^{1}(\tilde{X}, \tilde{\Sigma}, \mathbb{R})$ with

$$
\mathbf{j}(\lambda) \wedge \Re(\phi)=\mathbf{j}(\lambda) \wedge \Im(\phi)=0 .
$$


Then we have

$$
\|\lambda\|_{t} \leq e^{(1-\bar{\alpha}) t}\|\lambda\|_{0}
$$

where $\bar{\alpha}>0$ depends only on $\mathcal{K}$.

This theorem is essentially in [6] (Lemma 4.4). We reproduce the proof here for the convenience of the reader.

Proof of Theorem 7.2 Since $\mathcal{K}$ is compact, quadratic differentials in $\mathcal{K}$ have no short saddle connections. Hence, for $u \in[0, t], \mathbf{p}\left(q_{u}\right) \in \mathcal{K}$ implies that $\tilde{X}_{u}$ is thick (has no curves with short extremal lengths). Therefore, there exist a constant $\mathbf{c}_{\mathcal{K}}$ such that for any $u$ with $\mathbf{p}\left(q_{u}\right) \in \mathcal{K}$, any harmonic $\psi \in H^{1}\left(\tilde{X}_{u}, \mathbb{R}\right)$ and any $\operatorname{arc} \gamma$ on $\tilde{X}_{u}$ with end points in $\tilde{\Sigma}$,

$$
\left|\int_{\gamma} \psi\right| \leq \mathrm{c}_{\mathcal{K}}\|\psi\|_{H, u}\left(1+\ell_{u}(\gamma)\right)
$$

where $\ell_{u}(\gamma)$ is the length of $\gamma$ in flat metric associated to $\phi_{u}$.

Under the assumptions of Theorem 7.2, there exists $s \in[0.1 t, 0.9 t]$ such that $\mathbf{p}\left(q_{s}\right) \in \mathcal{K}$. Fix $p, p^{\prime} \in \Sigma$. Since $\tilde{X}_{0}$ is thick, there exists a path $\gamma_{0}$ connecting $p$ and $p^{\prime}$ with $\ell_{0}\left(\gamma_{0}\right)=O(1)$. Similarly, since $\tilde{X}_{s}$ and $\tilde{X}_{t}$ are thick there are paths $\gamma_{s}$ and $\gamma_{t}$ connecting $p$ and $p^{\prime}$ such that $\ell_{s}\left(\gamma_{s}\right)=O(1)$, $\ell_{t}\left(\gamma_{t}\right)=O(1)$. Then,

$$
\ell_{0}\left(\gamma_{s}\right)=O\left(e^{s}\right) \quad \text { and } \quad \ell_{s}\left(\gamma_{t}\right)=O\left(e^{t-s}\right) .
$$

Suppose $\lambda \in H^{1}(\tilde{X}, \tilde{\Sigma}, \mathbb{R})$ with $\mathbf{j}(\lambda) \wedge \Re(\phi)=\mathbf{j}(\lambda) \wedge \Im(\phi)=0$. Let $\psi=\mathbf{j}(\lambda)$. For $0 \leq u \leq t$, let $\psi_{u}$ denote the harmonic representative of the cohomology class $\psi$ on $\tilde{X}_{u}$. We have

$$
\begin{aligned}
\|\lambda\|_{t}-\|\lambda\|_{0} & \leq\|\psi\|_{H, t}-\|\psi\|_{H, 0}+\sum_{p, p^{\prime} \in \Sigma \times \Sigma}\left|\int_{\gamma_{p, p^{\prime}}} \psi_{t}-\psi_{0}\right| \\
& \leq e^{(1-\alpha) t}\|\psi\|_{H, 0}+\sum_{p, p^{\prime} \in \Sigma \times \Sigma}\left|\int_{\gamma_{p, p^{\prime}}} \psi_{t}-\psi_{0}\right|,
\end{aligned}
$$

where we have used Theorem 7.1. Since the integral in Eq. (60) is independent of the choice of $\gamma_{p, p^{\prime}}$, we use $\gamma_{p, p^{\prime}}=\gamma_{s}$. Then, by Eq. (59),

$$
\left|\int_{\gamma_{s}} \psi_{0}\right| \leq \mathrm{c}_{\mathcal{K}}\|\psi\|_{H, 0}\left(1+\ell_{0}\left(\gamma_{s}\right)\right) \stackrel{*}{\prec} \mathrm{c}_{\mathcal{K}}\|\psi\|_{H, 0} e^{s} .
$$


Also,

$$
\begin{aligned}
\left|\int_{\gamma_{s}} \psi_{t}\right| & =\left|\int_{\gamma_{s}-\gamma_{t}} \psi_{t}+\int_{\gamma_{t}} \psi_{t}\right| \\
& =\left|\int_{\gamma_{s}-\gamma_{t}} \psi_{s}+\int_{\gamma_{t}} \psi_{t}\right| \\
& \leq\left|\int_{\gamma_{s}} \psi_{s}\right|+\left|\int_{\gamma_{t}} \psi_{s}\right|+\left|\int_{\gamma_{t}} \psi_{t}\right| \\
& \stackrel{*}{\prec} \mathrm{c}_{\mathcal{K}}\left(\|\psi\|_{H, s}+\|\psi\|_{H, s} e^{t-s}+\|\psi\|_{H, t}\right) .
\end{aligned}
$$

where to pass from the first line to the second we used the fact that $\psi_{s}$ and $\psi_{t}$ represent the same cohomology class in $H^{1}(\tilde{X}, \mathbb{R})$, and in the last line we used Eq. (59) to estimate each term. Then, using Eq. (61), we have

$$
\begin{aligned}
\left|\int_{\gamma_{s}} \psi_{t}-\psi_{0}\right| & \stackrel{*}{\prec} \mathrm{c}_{\mathcal{K}}\left(\|\psi\|_{H, s}+\|\psi\|_{H, s} e^{t-s}+\|\psi\|_{H, t}+\|\psi\|_{H, 0} e^{s}\right) \\
& \leq \mathrm{c}_{\mathcal{K}}\left(e^{(1-\alpha) s}+e^{(1-\alpha) s+t-s}+e^{(1-\alpha) t}+e^{s}\right)\|\psi\|_{H, 0} \\
& \gtrless \mathrm{c}_{\mathcal{K}} e^{(1-0.1 \alpha) t}\|\psi\|_{H, 0},
\end{aligned}
$$

where in the second line we used Theorem 7.1 and in the last line we use the fact that $s \in[0.1 t, 0.9 t]$. Substituting into Eq. (60) we get

$$
\|\lambda\|_{t}-\|\lambda\|_{0} \leq \mathrm{c}_{\mathcal{K}} e^{(1-0.1 \alpha) t}\|\psi\|_{H, 0} \leq \mathrm{c}_{\mathcal{K}} e^{(1-0.1 \alpha) t}\|\lambda\|_{0} .
$$

Assuming $t$ is large enough, we can assume that the multiplicative error is less than $e^{\alpha_{0} t}$ for some $\alpha_{0} \leq 0.1 \alpha$. The theorem then holds for $\bar{\alpha} \leq\left(0.1 \alpha-\alpha_{0}\right)$.

\subsection{The Hodge distance}

Let $g_{t}$ be the Teichmüller flow on $\mathcal{Q}^{1} \mathcal{M}(\sigma)$. To each quadratic differential $q$, we associate its imaginary and real measured foliations $\eta^{-}(q)$, and $\eta^{+}(q)$.

The flow $g_{t}$ admits the following foliations:

1. $\mathcal{F}^{s s}$, whose leaves are sets of the form $\left\{(X, q) \mid \eta^{+}(q)=\right.$ const $\}$;

2. $\mathcal{F}^{u u}$, whose leaves are sets of the form $\left\{(X, q) \mid \eta^{-}(q)=\mathrm{const}\right\}$.

In other words, for $\left(X_{0}, q_{0}\right) \in \mathcal{Q}(\sigma)$, a leaf of $\mathcal{F}^{s s}$ is given by

$$
\alpha^{s s}\left(X_{0}, q_{0}\right)=\left\{(X, q) \in \mathcal{Q}(\sigma) \mid \eta^{+}(q)=\eta^{+}\left(q_{0}\right)\right\},
$$


and a leaf of $\mathcal{F}^{u u}$ is given by

$$
\alpha^{u u}\left(X_{0}, q_{0}\right)=\left\{(X, q) \in \mathcal{Q}(\sigma) \mid \eta^{-}(q)=\eta^{-}\left(q_{0}\right)\right\}
$$

Note that the foliations $\mathcal{F}^{s s}, \mathcal{F}^{u u}$ are invariant under both $g_{t}$ and $\Gamma(S)$; in particular, they descend to the moduli space $\mathcal{Q}^{1} \mathcal{M}(\sigma)$.

We also consider the foliation $\mathcal{F}^{u}$ whose leaves are defined by

$$
\alpha^{u}(q)=\bigcup_{t \in \mathbb{R}} g_{t} \alpha^{u u}(q)
$$

and $\mathcal{F}^{s}$ whose leaves are defined by

$$
\alpha^{s}(q)=\bigcup_{t \in \mathbb{R}} g_{t} \alpha^{s s}(q) .
$$

If $\mathcal{C}$ is a subset of moduli space of abelian differentials, we can locally identify a leaf of $\mathcal{F}^{s s}\left(\right.$ or $\left.\mathcal{F}^{u u}\right)$ with a subspace $W_{-}\left(\right.$or $\left.W^{+}\right)$of $H^{1}(X, \Sigma, \mathbb{R})$. In fact, for $\psi \in W_{-}\left(\right.$or $\left.\psi \in W^{+}\right)$, we have

$$
\mathbf{j}(\psi) \wedge \mathfrak{s}(\psi)=0 \text { and } \mathbf{j}(\psi) \wedge \mathfrak{R}(\psi)=0
$$

See $\S 1$ and $\S 2$ of [19] for more details.

If $\gamma$ is a map from $[0, r]$ into some leaf of $\mathcal{F}^{s s}$, then we define the Hodge length $\ell(\gamma)$ of $\gamma$ as $\int_{0}^{r}\left\|\gamma^{\prime}(t)\right\| d t$, where $\|\cdot\|$ is the Hodge norm. Finally:

- If two abelian differentials $\phi$ and $\phi^{\prime}$ belong to the same leaf of $\mathcal{F}^{s s}$, then we define $d_{H}\left(\phi, \phi^{\prime}\right)$ to be the infimum of $\ell(\gamma)$ where $\gamma$ varies over paths connecting $\phi$ and $\phi^{\prime}$ and staying in the leaf of $\mathcal{F}^{s s} \subset \mathcal{Q}(\sigma)$. We make the same definition if $\phi$ and $\phi^{\prime}$ are on the same leaf of $\mathcal{F}^{u u}$.

- By taking a ramified double cover (see Sect.7.2), we can define $d_{H}\left(q, q^{\prime}\right)$ for any $q, q^{\prime}$ on the same leaf of $\mathcal{F}^{s s}$ in $\mathcal{Q}(\sigma)$.

Lemma 7.3 Let $\mathcal{K}$ be a compact subset of $\mathcal{C}$. Suppose $(X, q),\left(X^{\prime}, q^{\prime}\right) \in$ $\mathbf{p}^{-1}(\mathcal{K})$ are in the same leaf of $\mathcal{F}^{s s}$. Let $\gamma$ be a Hodge length minimizing path connecting $q$ to $q^{\prime}$. Suppose $t>t_{0}$ is such that for all $q^{\prime \prime} \in \gamma$,

$$
\left\{s \in[0, t] \mid g_{s} q^{\prime \prime} \in \mathbf{p}^{-1}(\mathcal{K})\right\} \geq t / 2 .
$$

Then

$$
d_{H}\left(g_{t} q, g_{t} q^{\prime}\right) \leq e^{-c t} d_{H}\left(q, q^{\prime}\right)
$$

where c depend only on $\mathcal{K}$. 
Proof This follows from Theorem 7.2 and Eq. (62).

We now show that the above condition holds whenever the projections of $g_{t} q$ and $g_{t} q^{\prime}$ to $\mathcal{C}$ are also close. See also Lemma 5.4 of [16].

Lemma 7.4 Let $\mathcal{K}$ be a compact subset of $\mathcal{C}$. Then there is a larger compact subset $\mathcal{K}^{\prime} \subset \mathcal{C}$ and a covering of $\mathcal{K}$ with a finite family of open sets $\mathcal{U}$ so that the following holds. Let $U_{1}, U_{2} \subset \mathcal{Q}(\sigma)$ be connected open sets so that $\mathbf{p}\left(U_{i}\right) \in \mathcal{U}$, $i=1,2$. Let $(X, q),\left(X^{\prime}, q^{\prime}\right) \in U_{1}$ and $t>0$ be such that $g_{t}(q), g_{t}\left(q^{\prime}\right) \in U_{2}$. Further, assume that

$$
\left\{s \in[0, t] \mid \mathbf{p}\left(g_{s} q\right) \in \mathcal{K}\right\} \geq t / 2 .
$$

Then,

$$
\left\{s \in[0, t] \mid \mathbf{p}\left(g_{s} q^{\prime}\right) \in \mathcal{K}^{\prime}\right\} \geq t / 2 .
$$

Proof Let $\rho>0$. We can find an open cover $\mathcal{U}$ of $\mathcal{K}$ so that the following holds. Let $U$ be connected open sets so that $\mathbf{p}(U) \in \mathcal{U}$, and let $\left(X_{1}, q_{1}\right),\left(X_{2}, q_{2}\right) \in U$. Then for any saddle connection $\omega$, we have

$$
\frac{1}{\rho} \ell_{q_{1}}(\omega) \leq \ell_{q_{2}}(\omega) \leq \rho \ell_{q_{1}}(\omega)
$$

Let $U_{1}, U_{2} \subset \mathcal{Q}(\sigma)$ be connected open sets so that $\mathbf{p}\left(U_{i}\right) \in \mathcal{U}, i=1,2$. Let $(X, q),\left(X^{\prime}, q^{\prime}\right) \in U_{1}$ and $t>0$ be such that $g_{t}(q), g_{t}\left(q^{\prime}\right) \in U_{2}$. We first claim that (66) is true for quadratic differentials $q_{s}=g_{s}(q)$ and $q_{s}^{\prime}=g_{s}\left(q^{\prime}\right)$ as well for a larger constant $\rho^{\prime}=2 \rho$. Assume, for contradiction that

$$
\ell_{q_{s}}(\omega)>\rho^{\prime} \ell_{q_{s}^{\prime}}(\omega)
$$

for some $s \in[0, t]$. Assume $\omega$ is mostly vertical in $q_{s}$. That is,

$$
\Im\left(\operatorname{hol}_{q_{s}}(\omega)\right)>\frac{1}{2} \ell_{q_{s}}(\omega) .
$$

Then

$$
\begin{aligned}
\ell_{q}(\omega) & \geq \Im\left(\operatorname{hol}_{q}(\omega)\right) \\
& =e^{s} \Im\left(\operatorname{hol}_{q_{s}}(\omega)\right) \\
& >\frac{1}{2} e^{s} \ell_{q_{s}}(\omega) \\
& >\frac{1}{2} e^{s} \rho^{\prime} \ell_{q_{s}^{\prime}}(\omega) \geq \frac{1}{2} \rho^{\prime} \ell_{q^{\prime}}(\omega) .
\end{aligned}
$$


Which contradicts Eq. (66). In case $\omega$ is mostly horizontal, we move forward in time and argue the same way. This proves the claim.

Now let $\epsilon$ be such that the length of any saddle connection in $q \in \mathcal{K}$ is larger than $\epsilon$, and let $\mathcal{K}^{\prime}$ be the compact subset of $\mathcal{C}$ consisting of quadratic differentials where the length of every saddle connection is larger than $\epsilon^{\prime}=\epsilon / \rho^{\prime}$. Then (65) follows from the above length comparison.

Remark 7.5 We have essentially shown that under the assumption Eq. (64) we have exponential contraction along the foliation $\mathcal{F}^{s s}$ (and similarly exponential expansion along the foliation $\mathcal{F}^{u u}$ ).

\section{Outline of the proof of Theorem 1.2}

In this section, we prove Theorem 1.2. We only outline the arguments here since they are well known a more detailed version is already present in [22]. We essentially follow the work of Margulis [30]. First, we need a closing lemma.

Lemma 8.1 (Closing Lemma) Let $\mathcal{K}$ be a compact subset of $\mathcal{C}$ consisting of non-orbifold points. Given a quadratic differential $(x, q) \in \mathcal{K}$ and $\delta>0$, there exist constants $L_{0}>0$, and open neighborhoods $U \subset U^{\prime} \subset \mathcal{C}$ of $(x, q)$ with the following property. For $L>L_{0}$, suppose that $\mathbf{g}:[0, L] \rightarrow \mathcal{C}$ is a Teichmüller geodesic segment such that

(a) $\mathbf{g}(0), \mathbf{g}(L) \in U$ and

(b) $\mathbf{g}$ spends more than half of its length in $\mathcal{K}$.

Let $\mathbf{g}_{1}$ be the closed path in $\mathcal{C}$ which is the union of $\mathbf{g}$ and a segment connecting $\mathbf{g}(L)$ to $\mathbf{g}(0)$ in $U$. Then there exists a unique closed geodesic $\mathbf{g}^{\prime} \subset \mathcal{C}$ with the following properties:

(I) $\mathbf{g}^{\prime}$ and $\mathbf{g}_{1}$ have lifts in $\mathcal{T}(S)$ which stay $\delta$-close with respect to the Teichmüller metric.

(II) The length of $\mathbf{g}^{\prime}$ is within $\delta$ of $L$, (III) $\mathbf{g}^{\prime}$ passes through $U^{\prime}$.

Remark 8.2 We remark that in Lemma 8.1 if we remove the assumption that $\mathcal{K}$ consists of non-orbifold points then there are at most a uniformly bounded number of closed geodesics satisfying conditions (I-III). A version of the closing lemma can be found in [22].

Outline of the proof of Lemma 8.1 Consider the stable and unstable foliations for the geodesic flow. Our goal is to show that if $U$ is small enough, the first return map on these foliations will define a contraction with respect to the Hodge distance function. As a result, we find a fixed point for the first return map in $U^{\prime}$; this is the same as a closed geodesic going through $U^{\prime}$. 
In view of Lemma 7.3 and Lemma 7.4 there is in fact a neighborhood of $(x, q)$ such that the time $L$ geodesic flow restricted to the neighborhood expands along the leaves of $\mathcal{F}^{u u}$ and contracts along the leaves of $\mathcal{F}^{s s}$.

Then, the contraction mapping principle (applied first to the map on $\mathcal{F}^{s s}$ and then to the inverse of the map on $\mathcal{F}^{u u}$ ) allows us to find a fixed point for the geodesic flow near $(x, q)$ (in a slightly bigger neighboorhood). In other words, there are neighborhoods $U \subset U^{\prime}$ of $(x, q)$ such that:

- if $\mathbf{g}:[0, L] \rightarrow \mathcal{C}$ satisfies properties $(a)$ and $(b)$ then in view of the hyperbolicity statement (Lemma 7.3)

the time $L$ geodesic flow restricted to $U$ expands along the leaves of $\mathcal{F}^{u u}$ and contracts along the leaves of $\mathcal{F}^{s s}$, in the metric $d_{H}$,

- for any $q_{1}, q_{2} \in U$, if $q_{1} \in \mathcal{F}^{s s}\left(q_{2}\right)$ or $q_{1} \in \mathcal{F}^{u u}\left(q_{2}\right)$ then $d_{H}\left(q_{1}, q_{2}\right) \leq \delta$.

We can apply the contraction mapping principle to $\mathcal{F}^{s s}$ to find $\left(x_{0}, q_{0}\right) \in U^{\prime}$ such that $g_{L}\left(q_{0}\right) \in \mathcal{F}^{u u} q_{0}$. Now we can consider the first return map of the map $g_{-t}$ on $\mathcal{F}^{u u}\left(q_{0}\right)$.

Proof of Theorem 1.2 Note that by the bound proved in Theorem 1.5, we only need to consider the set of closed geodesics going through a fixed compact subset of $\mathcal{C}$. We have

- by Theorem 2.4, the geodesic flow on $\mathcal{C}$ is mixing, and

- on a fixed compact subset of $\mathcal{Q}^{1} \mathcal{M}(S, \sigma)$ the geodesic flow is uniformly hyperbolic.

- every nearly closed orbit approximates a close orbit (Lemma 8.1).

Hence, all the ingredients are in place to drive Theorem 1.2 following the work of Margulis [30]. (See also \$20.6 in [27].)

Acknowledgements We would like to thanks the referee for many useful comments that have improve the exposition of the paper at several places.

\section{References}

1. Arnoux, P., Yoccoz, J.: Construction de difféomorphismes pseudo-Anosov. C. R. Acad. Sci. Paris Sér. I Math. 292(1), 75-78 (1981)

2. Avila, A., Gouezel, S., Yoccoz, J.-C.: Exponential mixing for the Teichmüller flow. Publ. Math. IHES 104, 143-211 (2006)

3. Avila, A., João, R.M.: Exponential Mixing for the Teichmüller flow in the Space of Quadratic Differentials, preprint

4. Athreya, J.: Quantitative recurrence and large deviations for Teichmüller geodesic flow. Geom. Dedicata 119, 121-140 (2006)

5. Athreya, J., Bufetov, A., Eskin, A., Mirzakhani, M.: Lattice point asymptotics and volume growth on Teichmüller space. Duke Math. J. 161(6), 1055-1111 (2012)

6. Athreya, J., Forni, G.: Deviation of ergodic averages for rational polygonal billiards. Duke Math. J. 144(2), 285-319 (2008) 
7. Avila, A., Gouëzel, S.: Small eigenvalues of the Laplacian for algebraic measures in moduli space, and mixing properties of the Teichmüller flow. Ann. of Math. (2) 178(2), 385-442 (2013)

8. Avila, A., Gouëzel, S., Yoccoz, J.-C.: Exponential mixing for the Teichmüller flow. Publ. Math. Inst. Hautes Études Sci. 104, 143-211 (2006)

9. Bers, L.: An extremal problem for quasiconformal maps and a theorem by Thurston. Acta Math. 141, 73-98 (1978)

10. Bleiler, S., Casson, A.: Automorphisms of Surfaces After Nielsen and Thurston. London Mathematical Society Student Texts, vol. 9. Cambridge University Press, Cambridge (1988)

11. Bufetov, A.: Logarithmic asymptotics for the number of periodic orbits of the Teichmueller flow on Veech's space of zippered rectangles. Mosc. Math. J. 9(2), 245-261 (2009)

12. Choi, Y., Series, C., Rafi, K.: Lines of minima and Teichmülcer geodesics. Geom. Funct. Anal. 18(3), 698-754 (2008)

13. Dumas, D.: Skinning Maps are Finite-to-one, preprint

14. Eskin, A., Margulis, G., Mozes, S.: Upper bounds and asymptotics in a quantitative version of the Oppenheim conjecture. Ann. of Math. (2) 147(1), 93-141 (1998)

15. Eskin, A., Masur, H.: Asymptotic formulas on flat surfaces. Ergodic Theory Dynam. Syst. 21, 443-478 (2001)

16. Eskin, A., Mirzakhani, M.: Counting closed geodesics in moduli space. J. Mod. Dyn. 1, 71-105 (2011)

17. Farb, B., Margalit, D.: A primer on mapping class groups. In: Princeton Mathematical Series, vol. 49 (2012)

18. Fathi, A., Laudenbach, F., Poénaru, V.: Travaux de Thurston sur les surfaces, Astérisque, 66 and 67 (1979)

19. Forni, G.: Deviation of ergodic averages for area-preserving flows on surfaces of higher genus. Ann. of Math. (2) 155(1), 1-103 (2002)

20. Gardiner, F., Masur, H.: Extremal length geometry of Teichmüller space. Complex Var. Theory Appl. 16(2-3), 209-237 (1991)

21. Hamenstädt, U.: Dynamics of the Teichmueller flow on compact invariant sets. J. Mod. Dyn. 4, 393-418 (2010)

22. Hamenstädt, U.: Bowen's construction for the Teichmüeller flow. J. Mod. Dyn. 7, 489-526 (2013)

23. Harer, J.L., Penner, R.C.: Combinatorics of Train Tracks. In: Annals of Mathematics Studies, vol. 125 (1992)

24. Hubbard, J.: Teichmüller Theory and Applications to Geometry, Topology, and Dynamics I, Matrix Editions (2006)

25. Ivanov, N.V., Coefficients of expansion of pseudo-Anosov homeomorphisms, Zap. Nauchn. Sem. Leningrad. Otdel. Mat. Inst. Steklov. (LOMI), 167: Issled. Topol. 6(111-116), 191 (1988)

26. Kontsevich, M., Zorich, A.: Connected components of spaces of Abelian differentials with prescribed singularities. Invent. Math. 153, 631-683 (2003)

27. Katok, A., Hasselblat, B.: Introduction to the Modern Theory of Dynamical Systems. Cambridge University Press, Cambridge (1995)

28. Kerckhoff, S.: The asymptotic geometry of Teichmüller space. Topology 19, 23-41 (1980)

29. Lanneau, E.: Connected components of the strata of the moduli spaces of quadratic diferentials with prescribed singularities. Ann. Sci. Ecole Norm. Sup. (4) 41, 1-56 (2008)

30. Margulis, G.A.: On Some Aspects of the Theory of Anosov Flows, Ph.D. Thesis (1970), Springer, Berlin (2003)

31. Maskit, B.: Comparison of hyperbolic and extremal lengths. Ann. Acad. Sci. Fenn. 10, 381-386 (1985)

32. Masur, H.: Interval exchange transformations and measured foliations. Ann. of Math. (2) 115(1), 169-200 (1982) 
33. Masur, H., Minsky, Y.: Geometry of the complex of curves. II. Hierarchical structure. Geom. Funct. Anal. 10(4): 902-974 (2000)

34. Masur, H., Smillie, J.: Hausdorff Dimension of Sets of Nonergodic Measured Foliations. Ann. Math. 134(3): 455-543 (1991)

35. Masur, H., Smillie, J.: Quadratic differentials with prescribed singularities and pseudoAnosov diffeomorphisms. Commentarii Mathematics Helevetici 68(1), 289-307 (1993)

36. Minsky, Y.: Extremal length estimates and product regions in Teichmüller space. Duke Math. J. 83(2), 249-286 (1996)

37. Papadopoulos, A., Penner, R.: A characterization of pseudo-Anosov foliations. Pac. J. Math. 130, 359-377 (1987)

38. Papadopoulos, A., Penner, R.: Enumerating pseudo-Anosov foliations. Pac. J. Math. 142(1), 159-173 (1990)

39. Penner, R.: A construction of pseudo-Anosov homeomorphisms. Trans. Am. Math. Soc. 310(1), 179-197 (1988)

40. Rafi, K.: A characterization of short curves of a Teichmüller geodesic. Geom. Topol. 9, 179-202 (2005)

41. Rafi, K.: A combinatorial model for the Teichmüller metric. Geom. Funct. Anal 17(3), 936-959 (2007)

42. Rafi, K.: Thick-thin decomposition of quadratic differentials. Math. Res. Lett. 14(2), 333341 (2007)

43. Rafi, K.: Hyperbolicity in Teichmüller space. Geom. Topol. 18-5, 3025-3053 (2014)

44. Rafi, K.: Closed geodesics in the thin part of moduli space. In preparation

45. Strebel, K.: Quadratic differentials. Ergebnisse der Math 5, Springer, Berlin (1984)

46. Thurston, W.: On the geometry and dynamics of diffeomorphisms of surfaces. Bull. Am. Math. Soc. (N.S.) 19(2), 417-431 (1988)

47. Thurston, W.: Shapes of polyhedra and triangulations of the sphere, (English summary) The Epstein birthday schrift, 511D549, Geom. Topol. Monogr., 1, Geom. Topol. Publ., Coventry (1998)

48. Veech, W.: The Teichmüller geodesic flow. Ann. Math. (2) 124(3), 441-530 (1986)

49. Vorobets, Y.: Periodic geodesics on generic translation surfaces, (English summary) Algebraic and topological dynamics, 205D258, Contemp. Math., 385. Am. Math. Soc., Providence, RI (2005) 